Review

\title{
Metal Complexes of Quinolone Antibiotics and Their Applications: An Update
}

\author{
Valentina Uivarosi \\ Department of General and Inorganic Chemistry, Faculty of Pharmacy, \\ Carol Davila University of Medicine and Pharmacy, 6 Traian Vuia St, Bucharest 020956, Romania; \\ E-Mail: uivarosi.valentina@umf.ro; Tel.: +4-021-318-0742; Fax: +4-021-318-0750
}

Received: 8 August 2013; in revised form: 2 September 2013 / Accepted: 2 September 2013 / Published: 11 September 2013

\begin{abstract}
Quinolones are synthetic broad-spectrum antibiotics with good oral absorption and excellent bioavailability. Due to the chemical functions found on their nucleus (a carboxylic acid function at the 3-position, and in most cases a basic piperazinyl ring (or another N-heterocycle) at the 7-position, and a carbonyl oxygen atom at the 4-position) quinolones bind metal ions forming complexes in which they can act as bidentate, as unidentate and as bridging ligand, respectively. In the polymeric complexes in solid state, multiple modes of coordination are simultaneously possible. In strongly acidic conditions, quinolone molecules possessing a basic side nucleus are protonated and appear as cations in the ionic complexes. Interaction with metal ions has some important consequences for the solubility, pharmacokinetics and bioavailability of quinolones, and is also involved in the mechanism of action of these bactericidal agents. Many metal complexes with equal or enhanced antimicrobial activity compared to the parent quinolones were obtained. New strategies in the design of metal complexes of quinolones have led to compounds with anticancer activity. Analytical applications of complexation with metal ions were oriented toward two main directions: determination of quinolones based on complexation with metal ions or, reversely, determination of metal ions based on complexation with quinolones.
\end{abstract}

Keywords: quinolones; metal complexes; applications

\section{Introduction}

The generic term "quinolone antibiotics" refers to a group of synthetic antibiotics with bactericidal effects, good oral absorption and excellent bioavailability [1,2]. Nalidixic acid (1-ethyl-1,4-dihydro-7- 
methyl-4-oxo-1,8-naphthyridine-3-carboxylic acid, Figure 1), the first compound of the series, was introduced in therapy in the 1960s [3].

Figure 1. Nalidixic acid.<smiles>CCn1cc(C(=O)O)c(=O)c2ccc(C)nc21</smiles>

The clinical use of nalidixic acid was limited by its narrow spectrum of activity. Several modifications were made on the basis nucleus in order to enlarge the antibacterial spectrum and to improve the pharmacokinetics properties, two of these considered as being major: introduction of a piperazine moiety or another N-heterocycles in the position 7 and introduction of a fluoride atom at the position 6. Thus, the new 4-quinolones, fluoroquinolones, have been discovered starting in the 1980s. Taking into account the chemical structure of the basis nucleus (Figure 2), the quinolone are classified in four groups (Table 1) [4-6].

Figure 2. The general structure of 4-quinolones.<smiles>[R][R]c1cc2c(=O)c(C(=O)O)n[Y4]([2H])c2[Y4]([R])c1[R]</smiles>

Table 1. Classes of quinolones based on chemical structure.

\begin{tabular}{|c|c|c|c|c|c|c|c|c|c|}
\hline $\begin{array}{l}\text { Quinolone } \\
\text { group/base } \\
\text { heterocycle }\end{array}$ & $\mathbf{X}_{1}$ & $\mathbf{X}_{2}$ & $\mathbf{X}_{3}$ & $\mathbf{R}_{1}$ & $\mathbf{R}_{2}$ & $\mathbf{R}_{\mathbf{3}}$ & $\mathbf{R}_{\mathbf{4}}$ & Representatives & Generation \\
\hline \multirow{4}{*}{$\begin{array}{c}\text { Naphthyridine } \\
\text { (8-aza-4-quinolone) }\end{array}$} & $\mathrm{CH}$ & $\mathrm{N}$ & $\mathrm{C}$ & $\mathrm{H}$ & $\mathrm{CH}_{3}$ & $\mathrm{C}_{2} \mathrm{H}_{5}$ & - & Nalidixic acid & First \\
\hline & $\mathrm{CH}$ & $\mathrm{N}$ & $\mathrm{C}$ & $\mathrm{F}$ & & $\mathrm{C}_{2} \mathrm{H}_{5}$ & - & Enoxacin & Second \\
\hline & $\mathrm{CH}$ & $\mathrm{N}$ & $\mathrm{C}$ & $\mathrm{F}$ & & & - & Gemifloxacin & Third \\
\hline & $\mathrm{CH}$ & $\mathrm{N}$ & $\mathrm{C}$ & $\mathrm{F}$ & & & - & Tosufloxacin & Third \\
\hline
\end{tabular}


Table 1. Cont.

\begin{tabular}{|c|c|c|c|c|c|c|c|c|c|}
\hline $\begin{array}{l}\text { Quinolone } \\
\text { group/base } \\
\text { heterocycle } \\
\end{array}$ & $\mathbf{X}_{1}$ & $\mathbf{X}_{2}$ & $\mathbf{X}_{3}$ & $\mathbf{R}_{1}$ & $\mathbf{R}_{\mathbf{2}}$ & $\mathbf{R}_{\mathbf{3}}$ & $\mathbf{R}_{4}$ & Representatives & Generation \\
\hline \multirow{2}{*}{$\begin{array}{c}\text { Pyridopyrimidine } \\
\text { (6,8-diaza-4- } \\
\text { quinolone) }\end{array}$} & $\mathrm{CH}$ & $\mathrm{N}$ & $\mathrm{N}$ & - & & $\mathrm{C}_{2} \mathrm{H}_{5}$ & - & Pipemidic acid & First \\
\hline & $\mathrm{CH}$ & $\mathrm{N}$ & $\mathrm{N}$ & - & & $\mathrm{C}_{2} \mathrm{H}_{5}$ & - & Piromidic acid & First \\
\hline $\begin{array}{c}\text { Cinnoline } \\
\text { (2-aza-4-quinolone) }\end{array}$ & $\mathrm{N}$ & $\mathrm{C}$ & $\mathrm{C}$ & & & $\mathrm{C}_{2} \mathrm{H}_{5}$ & $\mathrm{H}$ & Cinoxacin & First \\
\hline \multirow{16}{*}{$\begin{array}{c}\text { Quinoline } \\
\text { (4-oxo-1,4- } \\
\text { dihydroquinoline, } \\
\text { 4-quinolone) }\end{array}$} & $\mathrm{CH}$ & $\mathrm{C}$ & $\mathrm{C}$ & $\mathrm{H}$ & & $\mathrm{C}_{2} \mathrm{H}_{5}$ & $\mathrm{H}$ & Rosoxacin & First \\
\hline & $\mathrm{CH}$ & $\mathrm{C}$ & $\mathrm{C}$ & & & $\mathrm{C}_{2} \mathrm{H}_{5}$ & $\mathrm{H}$ & Oxolinic acid & First \\
\hline & $\mathrm{CH}$ & $\mathrm{C}$ & $\mathrm{C}$ & $\mathrm{F}$ & & & & Flumequine & First \\
\hline & $\mathrm{CH}$ & $\mathrm{C}$ & $\mathrm{C}$ & $\mathrm{F}$ & & $\mathrm{C}_{2} \mathrm{H}_{5}$ & $\mathrm{H}$ & Norfloxacin & Second \\
\hline & $\mathrm{CH}$ & $\mathrm{C}$ & $\mathrm{C}$ & $\mathrm{F}$ & & $\mathrm{C}_{2} \mathrm{H}_{5}$ & $\mathrm{H}$ & Pefloxacin & Second \\
\hline & $\mathrm{CH}$ & $\mathrm{C}$ & $\mathrm{C}$ & $\mathrm{F}$ & & & $\mathrm{H}$ & Ciprofloxacin & Second \\
\hline & $\mathrm{CH}$ & $\mathrm{C}$ & $\mathrm{C}$ & $\mathrm{F}$ & & & $\mathrm{H}$ & Enrofloxacin & Second \\
\hline & $\mathrm{CH}$ & $\mathrm{C}$ & $\mathrm{C}$ & $\mathrm{F}$ & & $\mathrm{C}_{2} \mathrm{H}_{5}$ & $\mathrm{~F}$ & Lomefloxacin & Second \\
\hline & $\mathrm{CH}$ & $\mathrm{C}$ & $\mathrm{C}$ & $\mathrm{F}$ & & & & Ofloxacin & Second \\
\hline & $\mathrm{CH}$ & $\mathrm{C}$ & $\mathrm{C}$ & $\mathrm{F}$ & & & & Levofloxacin & Third \\
\hline & $\mathrm{CH}$ & $\mathrm{C}$ & $\mathrm{C}$ & $\mathrm{F}$ & & & $\mathrm{F}$ & Sparfloxacin ${ }^{*}$ & Third \\
\hline & $\mathrm{CH}$ & $\mathrm{C}$ & $\mathrm{C}$ & $\mathrm{F}$ & & & $\mathrm{OCH}_{3}$ & Gatifloxacin & Third \\
\hline & $\mathrm{CH}$ & $\mathrm{C}$ & $\mathrm{C}$ & $\mathrm{F}$ & & $\lambda$ & $\mathrm{OCH}_{3}$ & Balofloxacin & Third \\
\hline & $\mathrm{CH}$ & $\mathrm{C}$ & $\mathrm{C}$ & $\mathrm{F}$ & & & $\mathrm{Cl}$ & Clinafloxacin & Fourth \\
\hline & $\mathrm{CH}$ & $\mathrm{C}$ & $\mathrm{C}$ & $\mathrm{F}$ & & & $\mathrm{Cl}$ & Sitafloxacin & Fourth \\
\hline & $\mathrm{CH}$ & $\mathrm{C}$ & $\mathrm{C}$ & $\mathrm{F}$ & & $\Lambda$ & $\mathrm{OCH}_{3}$ & Moxifloxacin & Fourth \\
\hline
\end{tabular}


Based on their antibacterial spectrum and their pharmacokinetic properties, the quinolones are classified in four generations [7-9] (Table 2).

Table 2. Generations of quinolones based on their antibacterial spectrum and pharmacokinetic properties.

\begin{tabular}{ll}
\hline Quinolone generation & Characteristic features \\
\hline First & Active against Gram negative bacteria. \\
& High protein binding. \\
& Short half life. \\
& Low serum and tissue concentrations. \\
& Uncomplicated urinary tract infection. \\
& Oral administration. \\
\hline Second & Class I (enoxacin, norfloxacin, lomefloxacin) \\
& Enhanced activity against Gram negative bacteria. \\
& Protein binding (50\%). \\
& Longer half life than the first generation. \\
& Moderate serum and tissue concentrations. \\
& Uncomplicated or complicated urinary tract infections. \\
& Oral administration. \\
\cline { 2 - 3 } & Class II (ofloxacin, ciprofloxacin) \\
& Enhanced activity against Gram negative bacteria. \\
& Atipical pathogens, Pseudomonas aeruginosa (ciprofloxacin). \\
& Protein binding (20\%-50\%). \\
& Moderate to long half life. \\
& Higher serum and tissue concentrations compared with class I. \\
Complicated urinary infections, gastroenteritis, prostatitis, \\
nosocomial infections. \\
Oral and iv administration. \\
Active against Gram negative and Gram positive bacteria. \\
Similar pharmacokinetic profile as for second generation (class II). \\
Similar indications and mode of administration. Consider for community \\
aquired pneumonia in hospitalized patients. \\
\hline Extended activity against Gram positive and Gram negative bacteria. \\
Active against anaerobes and atypical bacteria. \\
Oral and i.v. administration. \\
Consider for treatment of intraabdominal infections. \\
\hline Third & \\
\hline &
\end{tabular}

Quinolones are bactericidal agents that inhibit the replication and transcription of bacterial DNA, causing rapid cell death $[10,11]$. They inhibit two antibacterial key-enzymes, DNA-gyrase (topoisomerase II) and DNA topoisomerase IV. DNA-gyrase is composed of two subunits encoded as GyrA and GyrB, and its role is to introduce negative supercoils into DNA, thereby catalyzing the separation of daughter chromosomes. DNA topoisomerase IV is composed of four subunits, two ParC and two ParE subunits and it is responsible for decatenation of DNA thereby allowing segregation into two daughter cells [12,13]. Quinolones interact with the enzyme-DNA complex, forming a drug-enzyme-DNA complex that blocks progression and the replication process $[14,15]$. 
Older quinolones have greater activity against DNA-gyrase than against topoisomerase IV in Gram negative bacteria and greater activity against topoisomerase IV than against DNA-gyrase in Gram positive bacteria. Newer quinolones equally inhibit both enzymes [16-18].

\section{Chemical Properties of Quinolones Related to Complexation Process}

Most quinolone molecules are zwitterionic, based on the presence of a carboxylic acid function at the 3-position and a basic piperazinyl ring (or another N-heterocycle) at the 7-position. Both functions are weak and give a good solubility for the quinolones in acidic or basic media.

Protonation equilibria of quinolones have been studied in aqueous solution using potentiometry, ${ }^{1} \mathrm{H}$ NMR spectrometry and UV spectrophotometry [19,20]. For a quinolone molecule with the general structure depicted in Figure 3, two proton-binding sites can be identified. In solution, such a molecule exists in four microscopic protonation forms, two of the microspecies being protonation isomers.

Figure 3. Protonation scheme of a fluoroquinolone molecule with piperazine ring at the 7-position (adapted from [20-22]).

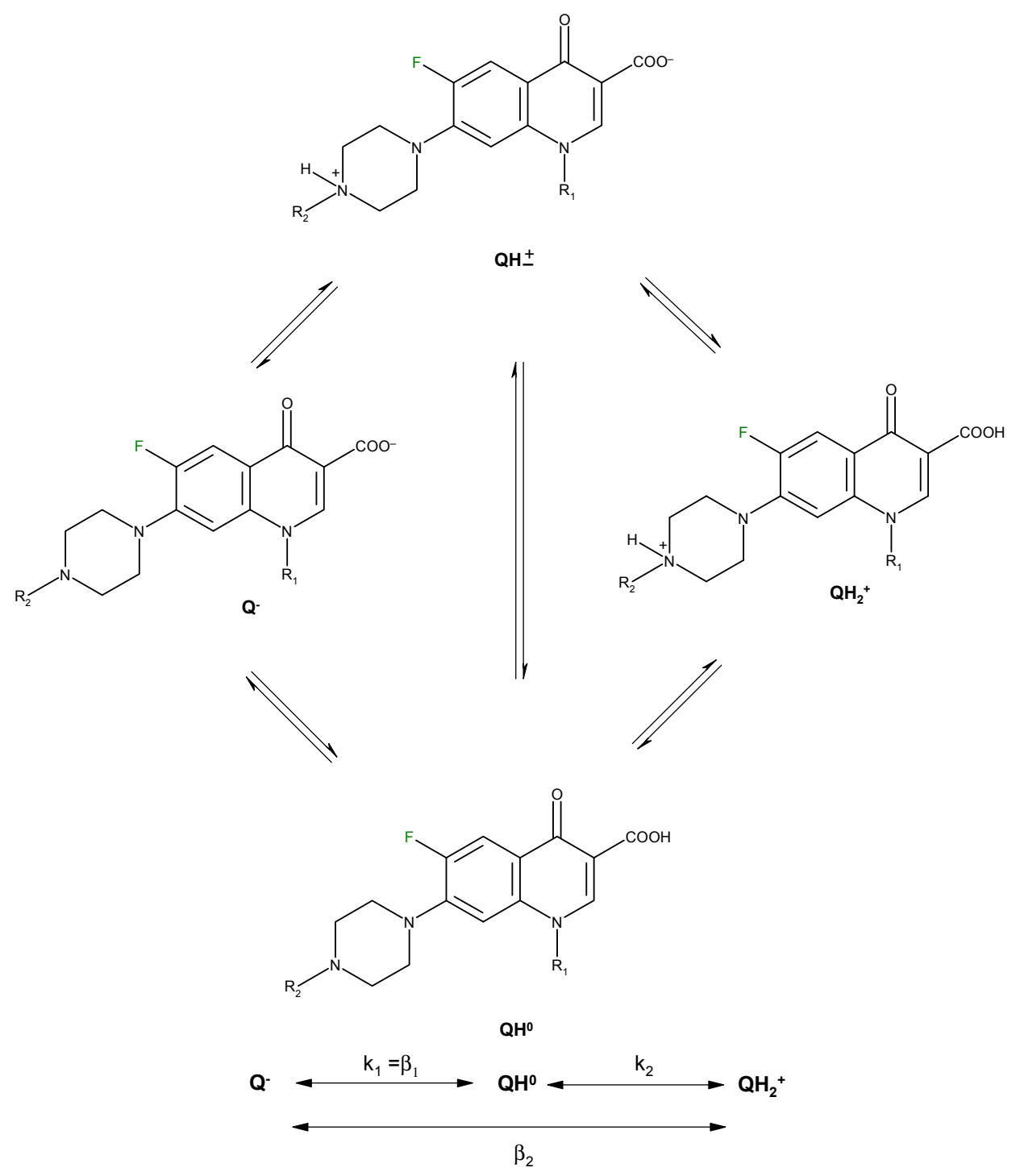


The microspeciation of drug molecules is used to depict the acid-base properties at the molecular level (macroconstants) and at the submolecular level (microconstants). The macroconstants quantify the overall basicity of the molecules. The values for $\mathrm{pKa}_{1}$, correlated with the acid function of carboxyl group, fall in the range 5.33-6.53, while the values for $\mathrm{pKa}_{2}$, correlated with the basic function of the piperazinic group, fall in the range 7.57-9.33. Table 3 contains the protonation constant values for norfloxacin and ofloxacin, two representative quinolones.

Table 3. Protonation constant values for norfloxacin and ofloxacin.

\begin{tabular}{cccccc}
\hline Compound & $\log \boldsymbol{\beta}_{\mathbf{1}}$ & $\log \boldsymbol{\beta}_{\mathbf{2}}=\log \mathbf{K a} \mathbf{a}_{\mathbf{2}}$ & $\log \boldsymbol{\beta}_{\mathbf{1}} \mathbf{-} \log \boldsymbol{\beta}_{\mathbf{2}}=\log \mathbf{K a} \mathbf{a}_{\mathbf{1}}$ & Isoelectric point & Reference \\
\hline Norfloxacin & 14.68 & 8.38 & 6.30 & 7.34 & {$[19]$} \\
& 14.73 & 8.51 & 6.22 & 7.37 & {$[23]$} \\
\hline \multirow{2}{*}{ Ofloxacin } & 14.27 & 8.22 & 6.05 & 7.14 & {$[19]$} \\
& 13.94 & 8.25 & 5.69 & 6.97 & {$[23]$} \\
\hline
\end{tabular}

The microconstants describe the proton binding affinity of the individual functional groups and are used in calculating the concentrations of different protonation isomers depending on the $\mathrm{pH}$. The quinolones exist mainly in the zwitterionic form between $\mathrm{pH} 3$ and 11 . The positively charged form $\mathrm{QH}_{2}^{+}$is present in $99.9 \%$ at $\mathrm{pH}$ 1. At $\mathrm{pH} 7.4$ all microspecies are present in commensurable concentrations.

Quinolone microspeciation has been correlated with bioavailability of quinolone molecules, serum protein binding and antibacterial activity [20]. The microspeciation is also important in the synthesis of metal complexes, the quinolone molecules acting as ligand in the deprotonated form ( $\mathrm{Q}^{-}$) in basic conditions, and in the zwitterionic form $\left(\mathrm{QH}^{ \pm}\right)$in neutral, slightly acidic or slightly basic medium. In strongly acidic medium, quinolones form ionic complexes in their cation form $\left(\mathrm{QH}_{2}{ }^{+}\right)$.

Quinolones form metal complexes due to their capacity to bind metal ions. In their metal complexes, the quinolones can act as bidentate ligand, as unidentate ligand and as bridging ligand. Frequently, the quinolones are coordinated in a bidentate manner, through one of the oxygen atoms of deprotonated carboxylic group and the ring carbonyl oxygen atom [Figure 4(a)]. Rarely, quinolones can act as bidentate ligand coordinated via two carboxyl oxygen atoms [Figure 4(b)] or through both piperazinic nitrogen atoms [Figure 4(c)]. Quinolones can also form complexes as unidentate ligand coordinated to the metal ion through by terminal piperazinyl nitrogen [Figure 4(d)]. In the polymeric complexes in solid state, multiple modes of coordination are simultaneously possible. In strongly acidic conditions quinolones are protonated and appear as cations in the ionic complexes.

Figure 4. Main coordination modes of quinolones.

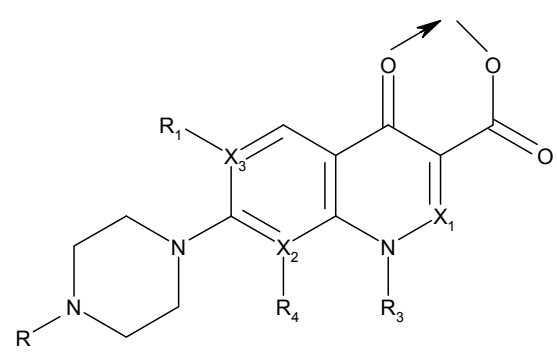

(a)<smiles></smiles>

(b) 
Figure 4. Cont.

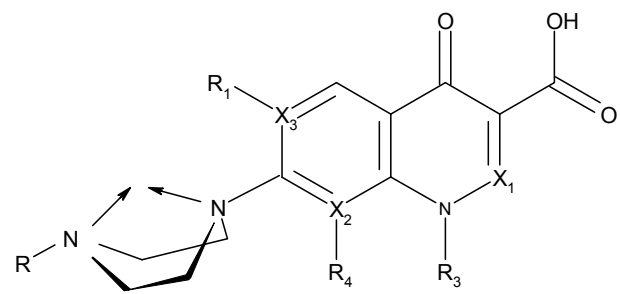

(c)

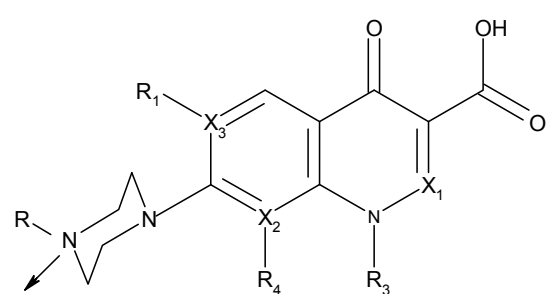

(d)

\section{Metal Complexes of Quinolones}

\subsection{Metal-Quinolone Chelates}

The quinolone molecules possess two main sites of metal chelate formation [Figures 4(a,c)]. The first of these, represented by the carbonyl and carboxyl groups in neighboring positions, is the most common coordination mode in the quinolone chelates. Quinolones can bind divalent cations $\left(\mathrm{Mg}^{2+}\right.$, $\mathrm{Ca}^{2+}, \mathrm{Cu}^{2+}, \mathrm{Zn}^{2+}, \mathrm{Fe}^{2+}, \mathrm{Co}^{2+}$ etc.), forming chelates with 1:1 or 1:2 (metal:ligand) stoichiometry or trivalent cations $\left(\mathrm{Al}^{3+}, \mathrm{Fe}^{3+}\right.$ ), forming chelates with $1: 1,1: 2$ or $1: 3$ (metal:ligand stoichiometry). A higher stoichiometry $(1: 4)$ is found in complexes with $\mathrm{Bi}^{3+}$. In Figure 5 is depicted the general structure of the chelates of quinolones with divalent cations with the 1:2 (metal:ligand) molar ratio. In a study of the $\mathrm{Cu}$ (II)-ciprofloxacin system it was observed that the number of coordinated ligands depends on the $\mathrm{pH}$. Thus, in the more acidic region, a 1:1 complex is favoured, whereas a 1:2 complex is the main species at higher $\mathrm{pH}$ values [24].

Figure 5. The general structure of $1: 2$ (metal:ligand) quinolone chelates with divalent cations.<smiles></smiles>

It was found that quinolones have a similar affinity for the metal ions, forming chelates more stable with hard Lewis acids like the trivalent cations $\left(\mathrm{Al}^{3+}, \mathrm{Fe}^{3+}\right)$. Chelates less stable are formed with the cations of group $2 \mathrm{~A}\left(\mathrm{Mg}^{2+}, \mathrm{Ca}^{2+}, \mathrm{Ba}^{2+}\right)$. For instance, the formation constant values for ciprofloxacin chelates decrease in order: $\mathrm{Al}^{3+}>\mathrm{Fe}^{3+}>\mathrm{Cu}^{2+}>\mathrm{Zn}^{2+}>\mathrm{Mn}^{2+}>\mathrm{Mg}^{2+}$ [25]. For norfloxacin chelates, the variation is quite similar: $\mathrm{Fe}^{3+}>\mathrm{Al}^{3+}>\mathrm{Cu}^{2+}>\mathrm{Fe}^{2+}>\mathrm{Zn}^{2+}>\mathrm{Mg}^{2+}>\mathrm{Ca}^{2+}$ [26]. 
The stability of chelates is greater in solvents with lower dielectric constant [26] and is $\mathrm{pH}$ dependent; the affinity of lomefloxacin for the $\mathrm{Ca}^{2+}$ and $\mathrm{Mg}^{2+}$ ions decreases in the order: anion $>$ zwitterion $>>$ cation [27].

Tables 4-6 present a selection of the chelates obtained in solid state with quinolone acting as bidentate ligand through the pyridone oxygen and one carboxylate oxygen, and the type of experiments carried out for investigating their biological activity. The tables include those chelates in which the quinolones are the only bidentate ligands; complexes with other bidentate co-ligands (e.g., 2, 2'-bipyridine, 1,10-phenantroline), and their biological activity are not discussed here.

Table 4. Selected chelates of quinolones from first generation.

\begin{tabular}{|c|c|c|c|c|c|}
\hline Ligand & $\begin{array}{l}\text { Metal } \\
\text { ion }\end{array}$ & $\begin{array}{c}\text { Molar ratio } \\
\text { M:L }\end{array}$ & $\begin{array}{l}\text { General formulae } \\
\text { of the complexes }\end{array}$ & $\begin{array}{l}\text { Complex tested/ } \\
\text { investigated for }\end{array}$ & Reference \\
\hline \multirow{11}{*}{$\begin{array}{l}\text { Pipemidic } \\
\text { acid }\end{array}$} & $\mathrm{VO}^{2+}$ & $1: 2$ & {$\left[\mathrm{VO}(\mathrm{PPA})_{2}\left(\mathrm{H}_{2} \mathrm{O}\right)\right]$} & DNA binding & [28] \\
\hline & $\mathrm{Mn}^{2+}$ & $1: 2$ & {$\left[\mathrm{Mn}(\mathrm{PPA})_{2}\left(\mathrm{H}_{2} \mathrm{O}\right)_{2}\right]$} & antimicrobial activity & \\
\hline & $\mathrm{Fe}^{3+}$ & $1: 3$ & {$\left[\mathrm{Fe}(\mathrm{PPA})_{3}\right]$} & & \\
\hline & $\mathrm{Co}^{2+}$ & $1: 2$ & {$\left[\mathrm{Co}(\mathrm{PPA})_{2}\left(\mathrm{H}_{2} \mathrm{O}\right)_{2}\right]$} & & \\
\hline & $\mathrm{Ni}^{2+}$ & $1: 2$ & {$\left[\mathrm{Ni}(\mathrm{PPA})_{2}\left(\mathrm{H}_{2} \mathrm{O}\right)_{2}\right]$} & & \\
\hline & $\mathrm{Zn}^{2+}$ & $1: 2$ & {$\left[\mathrm{Zn}(\mathrm{PPA})_{2}\left(\mathrm{H}_{2} \mathrm{O}\right)_{2}\right]$} & & \\
\hline & $\mathrm{MoO}_{2}^{2+}$ & $1: 2$ & {$\left[\mathrm{MoO}_{2}(\mathrm{PPA})_{2}\right]$} & & \\
\hline & $\mathrm{Cd}^{2+}$ & $1: 2$ & {$\left[\mathrm{Cd}(\mathrm{PPA})_{2}\left(\mathrm{H}_{2} \mathrm{O}\right)_{2}\right]$} & & \\
\hline & $\mathrm{UO}_{2}{ }^{2+}$ & $1: 2$ & {$\left[\mathrm{UO}_{2}(\mathrm{PPA})_{2}\right]$} & & \\
\hline & $\mathrm{Cu}^{2+}$ & $1: 2$ & {$\left[\mathrm{Cu}(\mathrm{PPA})_{2}\left(\mathrm{H}_{2} \mathrm{O}\right)\right]$} & $\begin{array}{c}\text { DNA binding } \\
\text { antimicrobial activity }\end{array}$ & [29] \\
\hline & $\mathrm{Fe}^{3+}$ & $1: 1$ & {$\left[\mathrm{Fe}(\mathrm{PPA})(\mathrm{HO})_{2}\left(\mathrm{H}_{2} \mathrm{O}\right)\right]_{2}$} & - & {$[30]$} \\
\hline \multirow[t]{9}{*}{ Cinoxacin } & $\mathrm{Cu}^{2+}$ & $1: 2$ & {$\left[\mathrm{Cu}(\mathrm{Cx})_{2}\left(\mathrm{H}_{2} \mathrm{O}\right)\right] \cdot 3 \mathrm{H}_{2} \mathrm{O}$} & - & [31] \\
\hline & $\mathrm{Ni}^{2+}$ & & {$\left[\mathrm{Ni}(\mathrm{Cx})_{2}(\mathrm{DMSO})_{2}\right] \cdot 4 \mathrm{H}_{2} \mathrm{O}$} & & \\
\hline & $\mathrm{Cu}^{2+}$ & $1: 2$ & {$\left[\mathrm{Cu}(\mathrm{Cx})_{2}\right] \cdot 2 \mathrm{H}_{2} \mathrm{O}$} & antimicrobial activity & [32] \\
\hline & $\mathrm{Co}^{2+}$ & $1: 3$ & {$\left[\mathrm{Co}(\mathrm{Cx})_{3}\right] \mathrm{Na} \cdot 10 \mathrm{H}_{2} \mathrm{O}$} & antimicrobial activity & [33] \\
\hline & $\mathrm{Cu}^{2+}$ & $1: 2$ & {$\left[\mathrm{Cu}(\mathrm{Cx})_{2}\right] \cdot 2 \mathrm{H}_{2} \mathrm{O}$} & & \\
\hline & & & $\mathrm{Cu}(\mathrm{Cx})(\mathrm{HCx}) \mathrm{Cl} \cdot 2 \mathrm{H}_{2} \mathrm{O}$ & & \\
\hline & $\mathrm{Zn}^{2+}$ & $1: 2$ & {$\left[\mathrm{Zn}(\mathrm{Cx})_{2}\right] \cdot 4 \mathrm{H}_{2} \mathrm{O}$} & & \\
\hline & $\mathrm{Cd}^{2+}$ & $1: 1$ & $\mathrm{Cd}(\mathrm{Cx}) \mathrm{Cl} \cdot \mathrm{H}_{2} \mathrm{O}$ & & \\
\hline & $\mathrm{Cd}^{2+}$ & $1: 3$ & $\begin{array}{c}\mathrm{Na}_{2}\left[\left(\mathrm{Cd}(\mathrm{Cx})_{3}\right)\left(\mathrm{Cd}(\mathrm{Cx})_{3}\left(\mathrm{H}_{2} \mathrm{O}\right)\right)\right] \\
12 \mathrm{H}_{2} \mathrm{O}\end{array}$ & - & [34] \\
\hline \multirow[t]{10}{*}{ Oxolinic acid } & $\mathrm{Cu}^{2+}$ & $1: 2$ & {$\left[\mathrm{Cu}(\text { oxo })_{2}\left(\mathrm{H}_{2} \mathrm{O}\right)\right]$} & $\begin{array}{c}\text { DNA binding } \\
\text { antimicrobial activity }\end{array}$ & [35] \\
\hline & $\mathrm{Ni}^{2+}$ & $1: 2$ & {$\left[\mathrm{Ni}(\mathrm{OxO})_{2}\left(\mathrm{H}_{2} \mathrm{O}\right)_{2}\right]$} & DNA binding & [36] \\
\hline & $\mathrm{Zn}^{2+}$ & $1: 2$ & {$\left[\mathrm{Zn}(\mathrm{oxo})_{2}\left(\mathrm{H}_{2} \mathrm{O}\right)_{2}\right]$} & DNA binding & [37] \\
\hline & $\mathrm{VO}^{2+}$ & $1: 2$ & {$\left[\mathrm{VO}(\mathrm{oxo})_{2}\left(\mathrm{H}_{2} \mathrm{O}\right)\right]$} & DNA binding & {$[38]$} \\
\hline & $\mathrm{Mn}^{2+}$ & $1: 2$ & {$\left[\mathrm{Mn}(\mathrm{oxo})_{2}\left(\mathrm{H}_{2} \mathrm{O}\right)_{2}\right]$} & & \\
\hline & $\mathrm{Fe}^{3+}$ & $1: 3$ & {$\left[\mathrm{Fe}(\mathrm{oxo})_{3}\right]$} & & \\
\hline & $\mathrm{Co}^{2+}$ & $1: 2$ & {$\left[\mathrm{Co}(\mathrm{oxo})_{2}\left(\mathrm{H}_{2} \mathrm{O}\right)_{2}\right]$} & & \\
\hline & $\mathrm{Ni}^{2+}$ & $1: 2$ & {$\left[\mathrm{Ni}(\mathrm{OxO})_{2}\left(\mathrm{H}_{2} \mathrm{O}\right)_{2}\right]$} & & \\
\hline & $\mathrm{Zn}^{2+}$ & $1: 2$ & {$\left[\mathrm{Zn}(\mathrm{oxo})_{2}\left(\mathrm{H}_{2} \mathrm{O}\right)_{2}\right]$} & & \\
\hline & $\mathrm{Cd}^{2+}$ & $1: 2$ & {$\left[\mathrm{Cd}(\mathrm{oxo})_{2}\left(\mathrm{H}_{2} \mathrm{O}\right)_{2}\right]$} & & \\
\hline
\end{tabular}


Table 4. Cont.

\begin{tabular}{cccccc}
\hline Ligand & $\begin{array}{c}\text { Metal } \\
\text { ion }\end{array}$ & $\begin{array}{c}\text { Molar ratio } \\
\text { M:L }\end{array}$ & $\begin{array}{c}\text { General formulae } \\
\text { of the complexes }\end{array}$ & $\begin{array}{c}\text { Complex tested/ } \\
\text { investigated for }\end{array}$ & Reference \\
\hline & $\begin{array}{c}\mathrm{MoO}_{2}{ }^{2+} \\
\mathrm{UO}_{2}{ }^{2+}\end{array}$ & $1: 2$ & $\begin{array}{c}{\left[\mathrm{MoO}_{2}(\mathrm{oxo})_{2}\right]} \\
{\left[\mathrm{UO}_{2}(\mathrm{oxo})_{2}\right]}\end{array}$ & $\begin{array}{c}\text { DNA binding } \\
\text { antimicrobial activity }\end{array}$ & {$[39]$} \\
\hline Flumequine & $\mathrm{Cu}^{2+}$ & $1: 2$ & $\begin{array}{c}{\left[\mathrm{Cu}(\mathrm{flmq})_{2}\left(\mathrm{OH}_{2}\right)_{2}\right]} \\
{\left[\mathrm{Zn}(\mathrm{flmq})_{2}\left(\mathrm{OH}_{2}\right)_{2}\right] \cdot \mathrm{H}_{2} \mathrm{O}}\end{array}$ & - & {$[40]$} \\
& $\mathrm{Zn}^{2+}$ & $1: 2$ & {$\left[\mathrm{Cu}(\mathrm{flmq})_{2}\left(\mathrm{H}_{2} \mathrm{O}\right)\right]$} & $\begin{array}{c}\text { DNA binding } \\
\text { albumin binding }\end{array}$ & {$[41]$} \\
\cline { 2 - 6 } & $\mathrm{Cu}^{2+}$ & $1: 2$ & {$\left[\mathrm{Ni}(\mathrm{flmq})_{2}\left(\mathrm{H}_{2} \mathrm{O}\right)_{2}\right]$} & $\begin{array}{c}\text { DNA binding } \\
\text { albumin binding }\end{array}$ & {$[42]$} \\
\cline { 2 - 6 } & $\mathrm{Ni}^{2+}$ & $1: 2$ & {$\left[\mathrm{Zn}(\mathrm{flmq})_{2}\left(\mathrm{H}_{2} \mathrm{O}\right)_{2}\right]$} & $\begin{array}{c}\text { DNA binding } \\
\text { albumin binding }\end{array}$ & {$[43]$} \\
\hline
\end{tabular}

Table 5. Selected chelates of quinolones from second generation.

\begin{tabular}{|c|c|c|c|c|c|}
\hline Ligand & Metal ion & $\begin{array}{c}\text { Molar } \\
\text { ratio M:L }\end{array}$ & $\begin{array}{l}\text { General formulae } \\
\text { of the complexes }\end{array}$ & $\begin{array}{l}\text { Complex tested/ } \\
\text { investigated for }\end{array}$ & Reference \\
\hline \multirow[t]{3}{*}{ Enoxacin } & $\mathrm{Co}^{2+}$ & $1: 2$ & $\begin{array}{l}{\left[\mathrm{Co}(\mathrm{HEx})_{2}\left(\mathrm{ClO}_{4}\right)_{2}\right] \cdot 3 \mathrm{H}_{2} \mathrm{O}} \\
{\left[\mathrm{Co}(\mathrm{HEx})_{2}\left(\mathrm{NO}_{3}\right)_{2}\right] \cdot 2 \mathrm{H}_{2} \mathrm{O}}\end{array}$ & $\begin{array}{c}\text { antimicrobial } \\
\text { activity } \\
\text { DNA oxidative } \\
\text { cleavage }\end{array}$ & [44] \\
\hline & $\begin{array}{l}\mathrm{Cu}^{2+} \\
\mathrm{Ni}^{2+} \\
\mathrm{Mn}^{2+} \\
\mathrm{Fe}^{3+} \\
\end{array}$ & $1: 2$ & $\begin{array}{c}{\left[\mathrm{M}(\mathrm{Ex})_{2}\left(\mathrm{H}_{2} \mathrm{O}\right)_{2}\right] \cdot 3 \mathrm{H}_{2} \mathrm{O}} \\
\left(\mathrm{M}=\mathrm{Cu}^{\mathrm{II}}, \mathrm{Ni}^{\mathrm{II}} \text { or } \mathrm{Mn}^{\mathrm{II}}\right) \\
\\
{\left[\mathrm{Fe}(\mathrm{Ex})\left(\mathrm{H}_{2} \mathrm{O}\right)_{2}\right] \mathrm{Cl} \cdot 4 \mathrm{H}_{2} \mathrm{O}}\end{array}$ & $\begin{array}{c}\text { antimicrobial } \\
\text { activity } \\
\text { antiinflammatory } \\
\text { activity } \\
\end{array}$ & [45] \\
\hline & $\mathrm{Ni}^{2+}$ & $1: 2$ & $\mathrm{Ni}(\mathrm{Ex})_{2} \cdot 2.5 \mathrm{H}_{2} \mathrm{O}$ & DNA binding & [46] \\
\hline \multirow[t]{9}{*}{ Norfloxacin } & $\begin{array}{l}\mathrm{Mg}^{2+} \\
\mathrm{Ca}^{2+} \\
\mathrm{Ba}^{2+} \\
\end{array}$ & $1: 2$ & 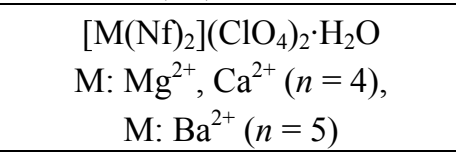 & - & [47] \\
\hline & $\mathrm{Al}^{3+}$ & $1: 3$ & {$\left[(\mathrm{Nf} \cdot \mathrm{HCl})_{3} \mathrm{Al}\right]$} & solubility behavior & {$[48]$} \\
\hline & $\mathrm{Bi}^{3+}$ & $1: 4$ & {$\left[\mathrm{Bi}\left(\mathrm{C}_{16} \mathrm{H}_{18} \mathrm{FN}_{3} \mathrm{O}_{3}\right)_{4}\left(\mathrm{H}_{2} \mathrm{O}\right)_{2}\right]$} & $\begin{array}{c}\text { antimicrobial } \\
\text { activity } \\
\text { solubility behavior }\end{array}$ & [49] \\
\hline & $\mathrm{Bi}^{3+}$ & $1: 3$ & {$\left[\mathrm{Bi}\left(\mathrm{C}_{16} \mathrm{H}_{17} \mathrm{FN}_{3} \mathrm{O}_{3}\right)_{3}\left(\mathrm{H}_{2} \mathrm{O}\right)_{2}\right]$} & $\begin{array}{c}\text { antimicrobial } \\
\text { activity, including } \\
\text { Helicobacter } \\
\text { pylori }\end{array}$ & [50] \\
\hline & $\mathrm{Mn}^{2+}$ & $1: 2$ & $\begin{array}{c}{\left[\mathrm{M}(\mathrm{Nf})_{2}\right] \mathrm{X}_{2} \cdot 8 \mathrm{H}_{2} \mathrm{O}} \\
\left(\mathrm{X}=\mathrm{CH}_{3} \mathrm{COO}^{-} \text {or } \mathrm{SO}_{4}{ }^{2-}\right) .\end{array}$ & - & [51] \\
\hline & $\mathrm{Fe}^{3+}$ & $1: 3$ & {$\left[\mathrm{Fe}(\mathrm{Nf})_{3}\right] \mathrm{Cl}_{3} \cdot 12 \mathrm{H}_{2} \mathrm{O}$} & - & \\
\hline & $\mathrm{Co}^{2+}$ & $1: 2$ & {$\left[\mathrm{Co}\left(\mathrm{NfH}-\mathrm{O}, \mathrm{O}^{\prime}\right)_{2}\left(\mathrm{H}_{2} \mathrm{O}\right)_{2}\right]\left(\mathrm{NO}_{3}\right)_{2}$} & - & [52] \\
\hline & $\begin{array}{l}\mathrm{Mn}^{2+} \\
\mathrm{Co}^{2+}\end{array}$ & $\begin{array}{l}1: 1 \\
1: 1\end{array}$ & $\begin{array}{l}{\left[\mathrm{MnCl}_{2}(\mathrm{Nf})\left(\mathrm{H}_{2} \mathrm{O}\right)_{2}\right]} \\
{\left[\mathrm{CoCl}_{2}(\mathrm{Nf})\left(\mathrm{H}_{2} \mathrm{O}\right)_{2}\right]}\end{array}$ & $\begin{array}{c}\text { biological } \\
\text { evaluation against } \\
\text { Trypanosoma } \\
\text { cruzi } \\
\end{array}$ & [53] \\
\hline & $\mathrm{Ni}^{2+}$ & $1: 2$ & {$\left[\mathrm{Ni}(\mathrm{Nf})_{2}\right] \cdot 6 \mathrm{H}_{2} \mathrm{O}$} & DNA binding & [46] \\
\hline
\end{tabular}


Table 5. Cont.

\begin{tabular}{|c|c|c|c|c|c|}
\hline Ligand & $\begin{array}{c}\text { Metal } \\
\text { ion }\end{array}$ & $\begin{array}{c}\text { Molar } \\
\text { ratio M:L }\end{array}$ & $\begin{array}{l}\text { General formulae } \\
\text { of the complexes }\end{array}$ & $\begin{array}{l}\text { Complex tested/ } \\
\text { investigated for }\end{array}$ & Reference \\
\hline & \multirow[t]{4}{*}{$\mathrm{Cu}^{2+}$} & $1: 2$ & $\mathrm{Cu}(\mathrm{HNf})_{2} \cdot 5 \mathrm{H}_{2} \mathrm{O}$ & - & \multirow[t]{3}{*}{ [54] } \\
\hline & & & {$\left[\mathrm{Cu}(\mathrm{HNf})_{2}\right] \mathrm{Cl}_{2} \cdot 2 \mathrm{H}_{2} \mathrm{O}$} & - & \\
\hline & & & $\mathrm{Cu}(\mathrm{HNf})_{2}\left(\mathrm{NO}_{3}\right)_{2} \cdot \mathrm{H}_{2} \mathrm{O}$ & - & \\
\hline & & $1: 2$ & {$\left[\mathrm{Cu}(\mathrm{NfH})_{2}\right] \mathrm{Cl}_{2} \cdot 6 \mathrm{H}_{2} \mathrm{O}$} & $\begin{array}{l}\text { DNA binding } \\
\text { albumin binding }\end{array}$ & [55] \\
\hline & $\mathrm{Zn}^{2+}$ & $1: 2$ & {$\left[\mathrm{Zn}(\mathrm{Nf})_{2}\right] \cdot 5 \mathrm{H}_{2} \mathrm{O}$} & - & {$[56]$} \\
\hline & $\mathrm{Zn}^{2+}$ & $1: 2$ & {$\left[\mathrm{M}(\mathrm{Nf})_{2}\right] \mathrm{X}_{2} \cdot \mathrm{nH}_{2} \mathrm{O}[\mathrm{M}=\mathrm{Zn}(\mathrm{II})$} & antimicrobial & [57] \\
\hline & $\mathrm{Cd}^{2+}$ & & $\left(\mathrm{X}=\mathrm{Cl}^{-}, \mathrm{CH}_{3} \mathrm{COO}^{-}, \mathrm{Br}^{-}\right.$and & activity & \\
\hline & $\mathrm{Hg}^{2+}$ & & $\begin{array}{c}\left.\mathrm{I}^{-}\right), \mathrm{Cd}(\mathrm{II}),\left(\mathrm{X}=\mathrm{Cl}^{-}, \mathrm{NO}_{3}^{-} \text {and }\right. \\
\left.\mathrm{SO}_{4}{ }^{2-}\right) \text { and } \mathrm{Hg}(\mathrm{II})\left(\mathrm{X}=\mathrm{Cl}^{-}\right. \\
\left.\left.\mathrm{NO}_{3}^{-} \text {and } \mathrm{CH}_{3} \mathrm{COO}^{-}\right)\right]\end{array}$ & & \\
\hline & $\mathrm{ZrO}^{2+}$ & $1: 2$ & {$\left[\mathrm{ZrO}(\mathrm{Nf})_{2} \mathrm{Cl}\right] \mathrm{Cl} \cdot 15 \mathrm{H}_{2} \mathrm{O}$} & antimicrobial & {$[58]$} \\
\hline & $\mathrm{UO}_{2}^{2+}$ & $1: 3$ & {$\left[\mathrm{UO}_{2}(\mathrm{Nf})_{3}\right]\left(\mathrm{NO}_{3}\right)_{2} \cdot 4 \mathrm{H}_{2} \mathrm{O}$} & activity & \\
\hline & $\mathrm{W}^{0}$ & & $\begin{array}{c}{\left[\mathrm{W}\left(\mathrm{H}_{2} \mathrm{O}\right)(\mathrm{CO})_{3}(\mathrm{H}-\mathrm{Nf})\right] \cdot} \\
(\mathrm{H}-\mathrm{Nf}) \cdot \mathrm{H}_{2} \mathrm{O}\end{array}$ & $\begin{array}{c}\text { antimicrobial } \\
\text { activity }\end{array}$ & [59] \\
\hline & $\mathrm{Ru}^{3+}$ & $1: 2$ & {$\left[\mathrm{Ru}(\mathrm{Nf})_{2} \mathrm{Cl}_{2}\right] \cdot 4 \mathrm{H}_{2} \mathrm{O}$} & - & [60] \\
\hline & $\mathrm{Pt}^{2+}$ & $1: 2$ & {$\left[\mathrm{Pt}(\mathrm{Nf})_{2}\right]$} & DNA binding & [61] \\
\hline & & & & $\begin{array}{l}\text { DNA cleavage } \\
\text { ability } \\
\text { antimicrobial } \\
\text { activity }\end{array}$ & \\
\hline & $\mathrm{Au}^{3+}$ & $1: 1$ & {$\left[\mathrm{AuCl}_{2}(\mathrm{Nf})\right] \mathrm{Cl}$} & $\begin{array}{c}\text { DNA binding } \\
\text { albumin binding } \\
\text { cytotoxic activity } \\
\text { cell cycle } \\
\end{array}$ & [62] \\
\hline & $\mathrm{Y}^{3+}$ & $1: 2$ & {$\left[\mathrm{Y}(\mathrm{Nf})_{2}\left(\mathrm{H}_{2} \mathrm{O}\right)_{2}\right] \mathrm{Cl}_{3} \cdot 10 \mathrm{H}_{2} \mathrm{O}$} & antimicrobial & [63] \\
\hline & $\mathrm{Pd}^{2+}$ & $1: 2$ & {$\left[\mathrm{Pd}(\mathrm{Nf})_{2}\right] \mathrm{Cl}_{2} \cdot 3 \mathrm{H}_{2} \mathrm{O}$} & activity & \\
\hline & $\mathrm{La}^{3+}$ & $1: 3$ & {$\left[\mathrm{La}(\mathrm{Nf})_{3}\right] \cdot 3 \mathrm{H}_{2} \mathrm{O}$} & antimicrobial & [64] \\
\hline & $\mathrm{Ce}^{3+}$ & $1: 3$ & {$\left[\mathrm{Ce}(\mathrm{Nf})_{3}\right] \cdot 3 \mathrm{H}_{2} \mathrm{O}$} & activity & \\
\hline & $\mathrm{Ln}=$ & $1: 4$ & {$\left[\mathrm{~N}\left(\mathrm{CH}_{3}\right)_{4}\right]\left[\mathrm{Ln}(\mathrm{Nf})_{4}\right] \cdot 6 \mathrm{H}_{2} \mathrm{O}$} & interaction with & [65] \\
\hline & $\mathrm{Nd}(\mathrm{III})$ & & & DNA and albumin & \\
\hline & $\mathrm{Sm}(\mathrm{III})$ & & & & \\
\hline & Ho(III) & & & & \\
\hline \multirow[t]{9}{*}{ Pefloxacin } & $\mathrm{Bi}^{3+}$ & $1: 3$ & {$\left[\mathrm{Bi}\left(\mathrm{C}_{17} \mathrm{H}_{19} \mathrm{FN}_{3} \mathrm{O}_{3}\right)_{3}\left(\mathrm{H}_{2} \mathrm{O}\right)_{2}\right]$} & antimicrobial & {$[50]$} \\
\hline & & & & activity, including & \\
\hline & & & & Helicobacter & \\
\hline & & & & pylori & \\
\hline & $\mathrm{Zn}^{2+}$ & $1: 2$ & {$\left[\mathrm{Zn}(\mathrm{Pf})_{2}\left(\mathrm{H}_{2} \mathrm{O}\right)\right] \cdot 2 \mathrm{H}_{2} \mathrm{O}$} & - & {$[66]$} \\
\hline & $\mathrm{Pt}^{2+}$ & $1: 2$ & {$\left[\mathrm{Pt}(\mathrm{Pf})_{2}\right]$} & DNA binding & [61] \\
\hline & & & & DNA cleavage & \\
\hline & & & & ability & \\
\hline & & & & $\begin{array}{l}\text { antimicrobial } \\
\text { activity }\end{array}$ & \\
\hline \multirow[t]{2}{*}{ Ciprofloxacin } & $\mathrm{Mg}^{2+}$ & $1: 2$ & {$\left[\mathrm{Mg}(\mathrm{Cf})_{2}\right] \cdot 2.5 \mathrm{H}_{2} \mathrm{O}$} & DNA binding & [67] \\
\hline & $\mathrm{Mg}^{2+}$ & $1: 2$ & {$\left[\mathrm{Mg}(\mathrm{Cf})_{2}\left(\mathrm{H}_{2} \mathrm{O}\right)_{2}\right] \cdot 2 \mathrm{H}_{2} \mathrm{O}$} & $\begin{array}{l}\text { antimicrobial } \\
\text { activity }\end{array}$ & [68] \\
\hline
\end{tabular}


Table 5. Cont.

\begin{tabular}{|c|c|c|c|c|c|}
\hline \multirow[t]{2}{*}{ Ligand } & Metal ion & $\begin{array}{c}\text { Molar } \\
\text { ratio M:L }\end{array}$ & $\begin{array}{l}\text { General formulae } \\
\text { of the complexes }\end{array}$ & $\begin{array}{l}\text { Complex tested/ } \\
\text { investigated for }\end{array}$ & Reference \\
\hline & \multirow[t]{2}{*}{$\mathrm{Mg}^{2+}$} & $1: 2$ & {$\left[\mathrm{Mg}\left(\mathrm{H}_{2} \mathrm{O}\right)_{2}(\mathrm{CfH})_{2}\right]\left(\mathrm{NO}_{3}\right)_{2} \cdot 2 \mathrm{H}_{2} \mathrm{O}$} & \multirow[t]{2}{*}{-} & \multirow[t]{2}{*}{ [69] } \\
\hline & & $1: 3$ & {$\left[\mathrm{Mg}(\mathrm{CfH})_{3}\right]\left(\mathrm{SO}_{4}\right) \cdot 5 \mathrm{H}_{2} \mathrm{O}$} & & \\
\hline & \multirow[t]{4}{*}{$\mathrm{Mg}^{2+} \mathrm{Ca}^{2+} \mathrm{Ba}^{2+}$} & \multirow[t]{4}{*}{$1: 2$} & {$\left[\mathrm{M}(\mathrm{Cf})_{2}\right]\left(\mathrm{ClO}_{4}\right)_{2} \cdot \mathrm{H}_{2} \mathrm{O}$} & \multirow[t]{4}{*}{-} & \multirow{4}{*}{$\begin{array}{l}{[47]} \\
{[70]}\end{array}$} \\
\hline & & & $\mathrm{M}: \mathrm{Mg}^{2+}(n=6)$ & & \\
\hline & & & $\mathrm{M}: \mathrm{Ca}^{2+}(n=4)$ & & \\
\hline & & & $\mathrm{M}: \mathrm{Ba}^{2+}(n=2)$ & & \\
\hline & \multirow{3}{*}{$\begin{array}{c}\mathrm{Mg}^{2+} \mathrm{Zn}^{2+} \\
\mathrm{Co}^{2+}\end{array}$} & $1: 2$ & {$\left[\mathrm{Mg}(\mathrm{Cf})_{2}\left(\mathrm{H}_{2} \mathrm{O}\right)_{2}\right] \cdot 2 \mathrm{H}_{2} \mathrm{O}$} & \multirow[t]{3}{*}{-} & \multirow[t]{3}{*}[22]{} \\
\hline & & & {$\left[\mathrm{Zn}(\mathrm{Cf})_{2}\right] \cdot 3 \mathrm{H}_{2} \mathrm{O}$} & & \\
\hline & & & {$\left[\mathrm{Co}(\mathrm{Cf})_{2}\right] \cdot 3 \mathrm{H}_{2} \mathrm{O}$} & & \\
\hline & $\mathrm{Al}^{3+}$ & $1: 3$ & {$\left[(\mathrm{Cf} \cdot \mathrm{HCl})_{3} \mathrm{Al}\right]$} & & [48] \\
\hline & \multirow[t]{4}{*}{$\mathrm{Bi}^{3+}$} & \multirow[t]{4}{*}{$1: 3$} & \multirow[t]{4}{*}[\mathrm{Bi}(\mathrm{C}_{17}\mathrm{H}_{17}\mathrm{FN}_{3}\mathrm{O}_{3})_{3}(\mathrm{H}_{2}\mathrm{O})_{2}]{} & antimicrobial & \multirow[t]{4}{*}{ [50] } \\
\hline & & & & activity, including & \\
\hline & & & & Helicobacter & \\
\hline & & & & pylori & \\
\hline & $\mathrm{VO}^{2+}$ & $1: 2$ & {$\left[\mathrm{VO}(\mathrm{Cf})_{2}\left(\mathrm{H}_{2} \mathrm{O}\right)\right]$} & - & [71] \\
\hline & \multirow{6}{*}{$\begin{array}{l}\mathrm{Mn}^{2+} \mathrm{Co}^{2+} \mathrm{Ni}^{2+} \\
\mathrm{Cu}^{2+} \mathrm{Zn}^{2+} \mathrm{Cd}^{2+}\end{array}$} & \multirow[t]{6}{*}{$1: 1$} & {$\left[\mathrm{Mn}(\mathrm{Cf})(\mathrm{OAc})\left(\mathrm{H}_{2} \mathrm{O}\right)_{2}\right] \cdot 3 \mathrm{H}_{2} \mathrm{O}$} & antimicrobial & \multirow[t]{6}{*}{ [72] } \\
\hline & & & {$\left[\mathrm{Co}(\mathrm{Cf})(\mathrm{OAc})\left(\mathrm{H}_{2} \mathrm{O}\right)_{2}\right] \cdot 3 \mathrm{H}_{2} \mathrm{O}$} & activity & \\
\hline & & & {$[\mathrm{Ni}(\mathrm{Cf})(\mathrm{OAc})] \cdot 6 \mathrm{H}_{2} \mathrm{O}$} & & \\
\hline & & & {$\left[\mathrm{Cu}(\mathrm{Cf})(\mathrm{OAc})\left(\mathrm{H}_{2} \mathrm{O}\right)_{2}\right] \cdot 3 \mathrm{H}_{2} \mathrm{O}$} & & \\
\hline & & & {$[\mathrm{Zn}(\mathrm{Cf})(\mathrm{OAc})] \cdot 6 \mathrm{H}_{2} \mathrm{O}$} & & \\
\hline & & & {$\left[\mathrm{Cd}(\mathrm{Cf})(\mathrm{OAc})\left(\mathrm{H}_{2} \mathrm{O}\right)_{2}\right] \cdot 3 \mathrm{H}_{2} \mathrm{O}$} & & \\
\hline & \multirow{5}{*}{$\begin{array}{c}\mathrm{Mn}^{2+} \mathrm{Fe}^{3+} \\
\mathrm{Co}^{2+} \\
\mathrm{Ni}^{2+} \mathrm{MoO}_{2}^{2+}\end{array}$} & \multirow{5}{*}{$\begin{array}{l}1: 2 \text { for } \mathrm{M}^{2+} \\
1: 3 \text { for } \mathrm{Fe}^{3+}\end{array}$} & {$\left[\mathrm{Mn}(\mathrm{Cf})_{2}\left(\mathrm{H}_{2} \mathrm{O}\right)_{2}\right]$} & DNA binding & \multirow[t]{5}{*}{ [73] } \\
\hline & & & {$\left[\mathrm{Fe}(\mathrm{Cf})_{3}\right]$} & & \\
\hline & & & {$\left[\mathrm{Co}(\mathrm{Cf})_{2}\left(\mathrm{H}_{2} \mathrm{O}\right)_{2}\right]$} & & \\
\hline & & & {$\left[\mathrm{Ni}(\mathrm{Cf})_{2}\left(\mathrm{H}_{2} \mathrm{O}\right)_{2}\right]$} & & \\
\hline & & & {$\left[\mathrm{MoO}_{2}(\mathrm{Cf})_{2}\right]$} & & \\
\hline & \multirow{5}{*}{$\begin{array}{c}\mathrm{Co}^{2+} \mathrm{Zn}^{2+} \mathrm{Cd}^{2+} \\
\mathrm{Ni}^{2+} \mathrm{Cu}^{2+}\end{array}$} & $1: 2$ & {$\left[\mathrm{Co}(\mathrm{Cf})_{2}\left(\mathrm{H}_{2} \mathrm{O}\right)\right] \cdot 9 \mathrm{H}_{2} \mathrm{O}$} & antimicrobial & [34] \\
\hline & & & {$\left[\mathrm{Zn}(\mathrm{Cf})_{2}\left(\mathrm{H}_{2} \mathrm{O}\right)_{2}\right] \cdot 8 \mathrm{H}_{2} \mathrm{O}$} & activity & \\
\hline & & & {$\left[\mathrm{Cd}(\mathrm{HCf})_{2}(\mathrm{Cl})_{2}\right] \cdot 4 \mathrm{H}_{2} \mathrm{O}$} & & \\
\hline & & & $\mathrm{M}(\mathrm{Cf})_{2} \cdot \mathrm{xH}_{2} \mathrm{O}$ & & \\
\hline & & & {$[\mathrm{M}=\mathrm{Ni}, \mathrm{Cu}, \mathrm{Cd}]$} & & \\
\hline & $\mathrm{Co}^{2+}$ & $1: 2$ & {$\left[\mathrm{Co}(\mathrm{Cf})_{2}\right] \cdot 3 \mathrm{H}_{2} \mathrm{O}$} & - & {$[22]$} \\
\hline & $\mathrm{Cu}^{2+}$ & $1: 2$ & $\left.\left[\mathrm{Cu}(\mathrm{HCf})_{2}\right]\left(\mathrm{NO}_{3}\right)_{2}\right] \cdot 6 \mathrm{H}_{2} \mathrm{O}$ & - & [74] \\
\hline & $\mathrm{Cu}^{2+}$ & $1: 2$ & {$\left[\mathrm{Cu}(\mathrm{Cf})_{2}\right] \mathrm{Cl}_{2} \cdot 11 \mathrm{H}_{2} \mathrm{O}$} & - & [75] \\
\hline & $\mathrm{Cu}^{2+}$ & $1: 2$ & {$\left[\mathrm{Cu}(\mathrm{Cf})_{2}\right] \mathrm{Cl}_{2} \cdot 6 \mathrm{H}_{2} \mathrm{O}$} & - & [76] \\
\hline & $\mathrm{Cu}^{2+}$ & $1: 2$ & {$\left[\mathrm{Cu}(\mathrm{HCf})_{2}\left(\mathrm{ClO}_{4}\right)_{2}\right] \cdot 6 \mathrm{H}_{2} \mathrm{O}$} & antimicrobial & [44] \\
\hline & & & {$\left[\mathrm{Cu}(\mathrm{HCf})_{2}\left(\mathrm{NO}_{3}\right)_{2}\right] \cdot 6 \mathrm{H}_{2} \mathrm{O}$} & activity & \\
\hline & & $1: 1$ & {$\left[\mathrm{Cu}(\mathrm{HCf})\left(\mathrm{C}_{2} \mathrm{O}_{4}\right)\right] \cdot 2 \mathrm{H}_{2} \mathrm{O}$} & DNA oxidative & \\
\hline & & & & cleavage & \\
\hline & $\mathrm{Cu}^{2+} /$ & $3: 2$ & {$\left[\mathrm{Cu}^{\mathrm{II}}(\mathrm{Cf})_{2}\left(\mathrm{Cu}^{\mathrm{I}} \mathrm{Cl}_{2}\right)_{2}\right]$} & antimicrobial & [77] \\
\hline & $\mathrm{Cu}^{+}$ & & & activity & \\
\hline & & & & Gyrase inhibition & \\
\hline & & & & DNA cleavage & \\
\hline & $\mathrm{Ru}^{3+}$ & $1: 2$ & {$\left[\mathrm{Ru}(\mathrm{Cf})_{2} \mathrm{Cl}_{2}\right] \mathrm{Cl} \cdot 3 \mathrm{H}_{2} \mathrm{O}$} & - & [60] \\
\hline & & $1: 3$ & {$\left[\mathrm{Ru}(\mathrm{Cf})_{3}\right] \cdot 4 \mathrm{H}_{2} \mathrm{O}$} & DNA interaction & [78] \\
\hline
\end{tabular}


Table 5. Cont.

\begin{tabular}{|c|c|c|c|c|c|}
\hline Ligand & $\begin{array}{c}\text { Metal } \\
\text { ion }\end{array}$ & $\begin{array}{c}\text { Molar } \\
\text { ratio M:L }\end{array}$ & $\begin{array}{l}\text { General formulae } \\
\text { of the complexes }\end{array}$ & $\begin{array}{l}\text { Complex tested/ } \\
\text { investigated for }\end{array}$ & Reference \\
\hline & $\mathrm{Pd}^{2+}$ & $1: 1$ & {$\left[\mathrm{PdCl}_{2}(\mathrm{~L})\right]$} & $\begin{array}{l}\text { antitubercular } \\
\text { activity }\end{array}$ & [79] \\
\hline & $\mathrm{Eu}^{3+}$ & $1: 2$ & $\begin{array}{c}\left.\mathrm{Eu}(\mathrm{CfH})(\mathrm{Cf})\left(\mathrm{H}_{2} \mathrm{O}\right)_{4}\right] \mathrm{Cl}_{2} \cdot \\
4.55 \mathrm{H}_{2} \mathrm{O}\end{array}$ & - & [80] \\
\hline \multirow[t]{13}{*}{ Lomefloxacin } & $\mathrm{Bi}^{3+}$ & $1: 3$ & {$\left[\mathrm{Bi}\left(\mathrm{C}_{17} \mathrm{H}_{18} \mathrm{~F}_{2} \mathrm{~N}_{3} \mathrm{O}_{3}\right)_{3}\left(\mathrm{H}_{2} \mathrm{O}\right)_{2}\right]$} & $\begin{array}{c}\text { antimicrobial } \\
\text { activity, including } \\
\text { H. pylori }\end{array}$ & {$[50]$} \\
\hline & $\mathrm{Y}^{3+}$ & $1: 2$ & {$\left[\mathrm{Y}(\mathrm{LFX})_{2} \mathrm{Cl}_{2}\right] \mathrm{Cl} \cdot 12 \mathrm{H}_{2} \mathrm{O}$} & antimicrobial & [81] \\
\hline & $\mathrm{ZrO}^{2+}$ & $1: 2$ & {$\left[\mathrm{ZrO}(\mathrm{LFX})_{2} \mathrm{Cl}\right] \mathrm{Cl} \cdot 15 \mathrm{H}_{2} \mathrm{O}$} & activity & \\
\hline & $\mathrm{UO}_{2}^{2+}$ & $1: 3$ & {$\left[\mathrm{UO}_{2}(\mathrm{LFX})_{3}\right]\left(\mathrm{NO}_{3}\right)_{2} \cdot 4 \mathrm{H}_{2} \mathrm{O}$} & & \\
\hline & $\mathrm{Cr}^{3+}$ & $1: 1$ & {$\left[\mathrm{Cr}(\mathrm{LFX})\left(\mathrm{H}_{2} \mathrm{O}\right)_{4}\right] \mathrm{Cl}_{3}$} & antimicrobial, & [82] \\
\hline & $\mathrm{Mn}^{2+}$ & $1: 1$ & {$\left[\mathrm{Mn}(\mathrm{LFX})\left(\mathrm{H}_{2} \mathrm{O}\right)_{4}\right] \mathrm{Cl}_{2}$} & antifungal, and & \\
\hline & $\mathrm{Fe}^{3+}$ & $1: 1$ & {$\left[\mathrm{Fe}(\mathrm{LFX})\left(\mathrm{H}_{2} \mathrm{O}\right)_{4}\right] \mathrm{Cl}_{3} \cdot \mathrm{H}_{2} \mathrm{O}$} & anticancer activity & \\
\hline & $\mathrm{Co}^{2+}$ & $1: 1$ & {$\left[\mathrm{Co}(\mathrm{LFX})\left(\mathrm{H}_{2} \mathrm{O}\right)_{4}\right] \mathrm{Cl}_{2}$} & & \\
\hline & $\mathrm{Ni}^{2+}$ & $1: 1$ & {$\left[\mathrm{Ni}(\mathrm{LFX})\left(\mathrm{H}_{2} \mathrm{O}\right)_{4}\right] \mathrm{Cl}_{2} \cdot \mathrm{H}_{2} \mathrm{O}$} & & \\
\hline & $\mathrm{Cu}^{2+}$ & $1: 1$ & {$\left[\mathrm{Cu}(\mathrm{LFX})\left(\mathrm{H}_{2} \mathrm{O}\right)_{4}\right] \mathrm{Cl}_{2} \cdot 2 \mathrm{H}_{2} \mathrm{O}$} & & \\
\hline & $\mathrm{Zn}^{2+}$ & $1: 1$ & {$\left[\mathrm{Zn}(\mathrm{LFX})\left(\mathrm{H}_{2} \mathrm{O}\right)_{4}\right] \mathrm{Cl}_{2}$} & & \\
\hline & $\operatorname{Th}(\mathrm{IV})$ & $1: 1$ & {$\left[\mathrm{Th}(\mathrm{LFX})\left(\mathrm{H}_{2} \mathrm{O}\right)_{4}\right] \mathrm{Cl}_{4}$} & & \\
\hline & $\mathrm{UO}_{2}^{2+}$ & $1: 1$ & {$\left[\mathrm{UO}_{2}(\mathrm{LFX})\left(\mathrm{H}_{2} \mathrm{O}\right)_{2}\right]\left(\mathrm{NO}_{3}\right)_{2}$} & & \\
\hline \multirow[t]{15}{*}{ Ofloxacin } & $\mathrm{Mg}^{2+}$ & $1: 2$ & $\begin{array}{c}{[\mathrm{Mg}(\mathrm{R}-\text { oflo })(\mathrm{S}-} \\
\left.\text { oflo })\left(\mathrm{H}_{2} \mathrm{O}\right)_{2}\right] \cdot 2 \mathrm{H}_{2} \mathrm{O}\end{array}$ & $\begin{array}{l}\text { antimicrobial } \\
\text { activity }\end{array}$ & [83] \\
\hline & $\mathrm{Ca}^{2+}$ & $1: 1$ & $\mathrm{Ca}$ (oflo) $\mathrm{Cl} \cdot 2 \mathrm{H}_{2} \mathrm{O}$ & - & [84] \\
\hline & $\mathrm{Mg}^{2+}$ & & $\mathrm{Mg}$ (oflo) $\mathrm{Cl} \cdot 2 \mathrm{H}_{2} \mathrm{O}$ & & \\
\hline & $\mathrm{Ba}^{2+}$ & & $\mathrm{Ba}$ (oflo) $\mathrm{Cl} \cdot 2 \mathrm{H}_{2} \mathrm{O}$ & & \\
\hline & $\mathrm{Ni}^{2+}$ & & $\mathrm{Ni}($ oflo $) \mathrm{Cl} \cdot 2 \mathrm{H}_{2} \mathrm{O}$ & & \\
\hline & $\mathrm{Co}^{2+}$ & & $\mathrm{Co}($ oflo $) \mathrm{Cl} \cdot 2 \mathrm{H}_{2} \mathrm{O}$ & & \\
\hline & $\mathrm{Zn}^{2+}$ & & $\mathrm{Zn}($ oflo $) \mathrm{Cl} \cdot \mathrm{H}_{2} \mathrm{O}$ & & \\
\hline & $\mathrm{Cu}^{2+}$ & $1: 2$ & {$\left[\mathrm{Cu}^{\mathrm{II}}(\mathrm{ofloH})_{2}\right]\left[\left(\mathrm{Cu}^{\mathrm{I}} \mathrm{Cl}_{2}\right)_{2}\right]$} & $\begin{array}{l}\text { DNA binding } \\
\text { albumin binding }\end{array}$ & {$[55]$} \\
\hline & $\begin{array}{l}\mathrm{Co}^{2+} \\
\mathrm{Zn}^{2+}\end{array}$ & $1: 2$ & {$\left[\mathrm{M}(\text { oflo })_{2}\right] \cdot 4 \mathrm{H}_{2} \mathrm{O}$} & - & [85] \\
\hline & $\mathrm{Cu}^{2+}$ & $1: 1$ & $\mathrm{M}($ oflo $) \mathrm{Cl} \cdot 2.5 \mathrm{H}_{2} \mathrm{O}$ & - & [86] \\
\hline & $\mathrm{Ni}^{2+}$ & & $\begin{array}{l}\mathrm{M}(\text { oflo })\left(\mathrm{SO}_{4}\right)_{0.5} \cdot 2.5 \mathrm{H}_{2} \mathrm{O} \\
\mathrm{M}(\mathrm{oflo})\left(\mathrm{NO}_{3}\right) \cdot 2.5 \mathrm{H}_{2} \mathrm{O}\end{array}$ & & \\
\hline & & $1: 2$ & $\begin{array}{c}{\left[\mathrm{Cu}(\text { oflo })_{2} \cdot \mathrm{H}_{2} \mathrm{O}\right] \cdot 2 \mathrm{H}_{2} \mathrm{O}} \\
\quad \mathrm{Ni}(\text { oflo })_{2} \cdot 3 \mathrm{H}_{2} \mathrm{O}\end{array}$ & & \\
\hline & $\mathrm{Pd}^{2+}$ & $1: 1$ & {$\left[\mathrm{PdCl}_{2}(\mathrm{~L})\right]$} & $\begin{array}{l}\text { antitubercular } \\
\text { activity }\end{array}$ & [79] \\
\hline & $\mathrm{Pt}^{2+}$ & $1: 2$ & {$\left[\mathrm{Pt}(\mathrm{oflo})_{2}\right]$} & $\begin{array}{l}\text { DNA binding } \\
\text { antimicrobial } \\
\text { activity }\end{array}$ & [61] \\
\hline & $\mathrm{Bi}^{3+}$ & $1: 3$ & {$\left[\mathrm{Bi}\left(\mathrm{C}_{17} \mathrm{H}_{17} \mathrm{FN}_{3} \mathrm{O}_{3}\right)_{3}\left(\mathrm{H}_{2} \mathrm{O}\right)_{2}\right]$} & $\begin{array}{c}\text { antimicrobial } \\
\text { activity, including } \\
\text { Helicobacter } \\
\text { pylori }\end{array}$ & {$[50]$} \\
\hline
\end{tabular}


Table 5. Cont.

\begin{tabular}{|c|c|c|c|c|c|}
\hline Ligand & Metal ion & $\begin{array}{c}\text { Molar } \\
\text { ratio M:L }\end{array}$ & $\begin{array}{l}\text { General formulae } \\
\text { of the complexes }\end{array}$ & $\begin{array}{l}\text { Complex tested/ } \\
\text { investigated for }\end{array}$ & Reference \\
\hline & $\begin{array}{l}\mathrm{Pr}^{3+} \\
\mathrm{Nd}^{3+}\end{array}$ & $1: 1$ & $\begin{array}{c}{\left[\mathrm{PrL}\left(\mathrm{NO}_{3}\right)_{2}\left(\mathrm{CH}_{3} \mathrm{OH}\right)\right]\left(\mathrm{NO}_{3}\right)} \\
{\left[\mathrm{NdL}\left(\mathrm{NO}_{3}\right)_{2}\left(\mathrm{CH}_{3} \mathrm{OH}\right)\right]\left(\mathrm{NO}_{3}\right)}\end{array}$ & $\begin{array}{l}\text { DNA binding } \\
\text { DNA cleavage } \\
\text { activity } \\
\text { antioxidation } \\
\text { properties }\end{array}$ & [87] \\
\hline \multirow[t]{8}{*}{ Enrofloxacin } & $\mathrm{VO}^{2+}$ & $1: 2$ & {$\left[\mathrm{VO}(\mathrm{erx})_{2}\left(\mathrm{H}_{2} \mathrm{O}\right)\right]$} & $\begin{array}{l}\text { antimicrobial } \\
\text { activity } \\
\text { DNA binding }\end{array}$ & [88] \\
\hline & $\mathrm{MO}_{2}{ }^{2+}$ & $1: 2$ & {$\left[\mathrm{MoO}_{2}(\mathrm{erx})_{2}\right]$} & $\begin{array}{l}\text { antimicrobial } \\
\text { activity } \\
\text { DNA binding }\end{array}$ & [89] \\
\hline & $\begin{array}{c}\mathrm{Mn}^{2+} \\
\mathrm{Fe}^{3+} \\
\mathrm{Co}^{2+} \\
\mathrm{Ni}^{2+} \mathrm{Zn}^{2+} \\
\mathrm{Cd}^{2+} \\
\mathrm{UO}_{2}{ }^{2+}\end{array}$ & $\begin{array}{l}1: 2 \text { for } \mathrm{M}^{2+}, \\
1: 3 \text { for } \mathrm{Fe}^{3+}\end{array}$ & $\begin{array}{c}{\left[\mathrm{Mn}(\mathrm{erx})_{2}\left(\mathrm{H}_{2} \mathrm{O}\right)_{2}\right]} \\
{\left[\mathrm{Fe}(\mathrm{erx})_{3}\right]} \\
{\left[\mathrm{Co}(\mathrm{erx})_{2}\left(\mathrm{H}_{2} \mathrm{O}\right)_{2}\right]} \\
{\left[\mathrm{Ni}(\mathrm{erx})_{2}\left(\mathrm{H}_{2} \mathrm{O}\right)_{2}\right]} \\
{\left[\mathrm{Zn}(\mathrm{erx})_{2}\left(\mathrm{H}_{2} \mathrm{O}\right)_{2}\right]} \\
{\left[\mathrm{Cd}(\mathrm{erx})_{2}\left(\mathrm{H}_{2} \mathrm{O}\right)_{2}\right]} \\
{\left[\mathrm{UO}_{2}(\mathrm{erx})_{2}\right]}\end{array}$ & $\begin{array}{l}\text { antimicrobial } \\
\text { activity } \\
\text { DNA binding }\end{array}$ & [90] \\
\hline & $\mathrm{Ni}^{2+}$ & $1: 2$ & {$\left[\mathrm{Ni}(\mathrm{erx})_{2}\left(\mathrm{H}_{2} \mathrm{O}\right)_{2}\right]$} & $\begin{array}{l}\text { DNA binding } \\
\text { albumin binding }\end{array}$ & [91] \\
\hline & $\mathrm{Cu}^{2+}$ & $1: 2$ & {$\left[\mathrm{Cu}(\operatorname{erx})_{2}\right] \mathrm{Cl}$} & $\begin{array}{c}\text { antimicrobial } \\
\text { activity }\end{array}$ & [92] \\
\hline & $\mathrm{Cu}^{2+}$ & $1: 2$ & {$\left[\mathrm{Cu}(\mathrm{erx})_{2}\left(\mathrm{H}_{2} \mathrm{O}\right)\right]$} & $\begin{array}{l}\text { DNA binding } \\
\text { antimicrobial } \\
\text { activity }\end{array}$ & [93] \\
\hline & $\mathrm{Cu}^{2+}$ & $1: 2$ & {$\left[\mathrm{Cu}(\mathrm{erx})_{2}\left(\mathrm{H}_{2} \mathrm{O}\right)_{2}\right]$} & - & [94] \\
\hline & $\mathrm{Ru}^{3+}$ & $1: 2$ & {$\left[\mathrm{Ru}(\mathrm{erx})_{2} \mathrm{Cl}_{2}\right] \mathrm{Cl} \cdot 5 \mathrm{H}_{2} \mathrm{O}$} & - & [60] \\
\hline
\end{tabular}

Table 6. Selected chelates of quinolones from third and fourth generation.

\begin{tabular}{|c|c|c|c|c|c|}
\hline Ligand & $\begin{array}{c}\text { Metal } \\
\text { ion }\end{array}$ & $\begin{array}{c}\text { Molar } \\
\text { ratio M:L }\end{array}$ & $\begin{array}{c}\text { General formulae } \\
\text { of the complexes }\end{array}$ & $\begin{array}{c}\text { Complex } \\
\text { tested/investigated for }\end{array}$ & Reference \\
\hline \multirow[t]{8}{*}{ Sparfloxacin } & $\mathrm{Bi}^{3+}$ & $1: 3$ & {$\left[\mathrm{Bi}\left(\mathrm{C}_{19} \mathrm{H}_{21} \mathrm{~F}_{2} \mathrm{~N}_{4} \mathrm{O}_{3}\right)_{3}\left(\mathrm{H}_{2} \mathrm{O}\right)_{2}\right]$} & $\begin{array}{c}\text { antimicrobial activity, } \\
\text { including Helicobacter } \\
\text { pylori }\end{array}$ & {$[50]$} \\
\hline & $\mathrm{Fe}^{3+}$ & $1: 31: 2$ for & {$\left[\mathrm{Fe}(\mathrm{sf})_{3}\right]$} & DNA binding & [95] \\
\hline & $\mathrm{VO}^{2+}$ & $\mathrm{M}^{2+}$ & {$\left[\mathrm{VO}(\mathrm{sf})_{2}\left(\mathrm{H}_{2} \mathrm{O}\right)\right]$} & Serum albumin binding & \\
\hline & $\mathrm{Mn}^{2+}$ & & {$\left[\mathrm{Mn}(\mathrm{sf})_{2}\left(\mathrm{H}_{2} \mathrm{O}\right)_{2}\right]$} & & \\
\hline & $\mathrm{Ni}^{2+}$ & & {$\left[\mathrm{Ni}(\mathrm{sf})_{2}\left(\mathrm{H}_{2} \mathrm{O}\right)_{2}\right]$} & & \\
\hline & $\mathrm{UO}_{2}{ }^{2+}$ & & {$\left[\mathrm{UO}_{2}(\mathrm{sf})_{2}\right]$} & & \\
\hline & $\mathrm{Co}^{2+}$ & $1: 2$ & {$\left[\mathrm{Co}(\mathrm{sf})_{2}\left(\mathrm{H}_{2} \mathrm{O}\right)_{2}\right]$} & $\begin{array}{l}\text { antimicrobial activity } \\
\text { DNA binding }\end{array}$ & [96] \\
\hline & $\mathrm{Cu}^{2+}$ & $1: 2$ & {$\left[\mathrm{Cu}(\mathrm{sf})_{2}\right]$} & $\begin{array}{c}\text { antimicrobial activity } \\
\text { DNA binding }\end{array}$ & [97] \\
\hline
\end{tabular}


Table 6. Cont.

\begin{tabular}{|c|c|c|c|c|c|}
\hline Ligand & $\begin{array}{l}\text { Metal } \\
\text { ion }\end{array}$ & $\begin{array}{c}\text { Molar } \\
\text { ratio M:L }\end{array}$ & $\begin{array}{l}\text { General formulae } \\
\text { of the complexes }\end{array}$ & $\begin{array}{c}\text { Complex } \\
\text { tested/investigated for }\end{array}$ & Reference \\
\hline & $\mathrm{Mn}^{2+}$ & $1: 1$ & {$\left[\mathrm{MnCl}_{2}(\mathrm{sf})\left(\mathrm{H}_{2} \mathrm{O}\right)_{2}\right]$} & biological evaluation & [53] \\
\hline & $\mathrm{Co}^{2+}$ & $1: 1$ & {$\left[\mathrm{CoCl}_{2}(\mathrm{sf})\left(\mathrm{H}_{2} \mathrm{O}\right)_{2}\right]$} & $\begin{array}{c}\text { against Trypanosoma } \\
\text { cruzi }\end{array}$ & \\
\hline & $\mathrm{MO}_{2}{ }^{2+}$ & $1: 2$ & {$\left[\mathrm{MoO}_{2}(\mathrm{sf})_{2}\right]$} & $\begin{array}{l}\text { antimicrobial activity } \\
\text { DNA binding }\end{array}$ & [89] \\
\hline & $\mathrm{Pd}^{2+}$ & $1: 1$ & {$\left[\mathrm{PdCl}_{2}(\mathrm{~L})\right]$} & antitubercular activity & [79] \\
\hline & $\mathrm{Pt}^{2+}$ & $1: 2$ & {$\left[\mathrm{Pt}(\mathrm{sf})_{2}\right]$} & $\begin{array}{l}\text { DNA binding } \\
\text { DNA cleavage ability } \\
\text { antimicrobial activity }\end{array}$ & [61] \\
\hline & $\mathrm{Au}^{3+}$ & $1: 1$ & {$\left[\mathrm{AuCl}_{2}(\mathrm{sf})\right] \mathrm{Cl}$} & $\begin{array}{c}\text { DNA binding } \\
\text { albumin binding } \\
\text { cytotoxic activity } \\
\text { cell cycle }\end{array}$ & [62] \\
\hline \multirow[t]{10}{*}{ Levofloxacin } & $\mathrm{Mg}^{2+}$ & $1: 2$ & {$\left[\mathrm{Mg}(\mathrm{S}-\text {-oflo })_{2}\left(\mathrm{H}_{2} \mathrm{O}\right)_{2}\right] \cdot 2 \mathrm{H}_{2} \mathrm{O}$} & antimicrobial activity & [83] \\
\hline & $\mathrm{Mn}^{2+}$ & $1: 2$ & {$\left[\mathrm{M}(\mathrm{levo})_{2}\left(\mathrm{H}_{2} \mathrm{O}\right)_{2}\right] \cdot \mathrm{nH}_{2} \mathrm{O}$} & antimicrobial activity & {$[98]$} \\
\hline & $\mathrm{Co}^{2+}$ & & $\left(n=2\right.$, excepting for $\mathrm{Cu}^{2+}$, & immunomodulatory & \\
\hline & $\mathrm{Ni}^{2+}$ & & $n=3)$ & activity & \\
\hline & $\mathrm{Cu}^{2+}$ & & & cytotoxicity & \\
\hline & $\mathrm{Zn}^{2+}$ & & & & \\
\hline & $\mathrm{Zn}^{2+}$ & $1: 2$ & {$\left[\mathrm{Zn}(\mathrm{levo})_{2}\left(\mathrm{H}_{2} \mathrm{O}\right)_{2}\right]$} & $\begin{array}{l}\text { DNA binding } \\
\text { albumin binding }\end{array}$ & [99] \\
\hline & $\mathrm{Pd}^{2+}$ & $1: 1$ & {$\left[\mathrm{PdCl}_{2}(\mathrm{~L})\right]$} & antitubercular activity & [79] \\
\hline & $\mathrm{Pt}^{2+}$ & $1: 2$ & {$\left[\mathrm{Pt}(\text { levo })_{2}\right]$} & $\begin{array}{c}\text { DNA binding } \\
\text { DNA cleavage ability } \\
\text { antimicrobial activity }\end{array}$ & [61] \\
\hline & $\mathrm{Au}^{3+}$ & $1: 1$ & {$\left[\mathrm{AuCl}_{2}(\right.$ levo $\left.)\right] \mathrm{Cl}$} & $\begin{array}{c}\text { DNA binding } \\
\text { albumin binding } \\
\text { cytotoxic activity } \\
\text { cell cycle }\end{array}$ & [62] \\
\hline \multirow[t]{10}{*}{ Gatifloxacin } & $\mathrm{Mg}^{2+}$ & $1: 2$ & {$\left[\mathrm{Mg}(\text { gat })_{2}\left(\mathrm{H}_{2} \mathrm{O}\right)_{2}\right] \mathrm{Cl}_{2} \cdot 2 \mathrm{H}_{2} \mathrm{O}$} & antimicrobial activity & {$[100]$} \\
\hline & $\mathrm{Ca}^{2+}$ & & {$\left[\mathrm{Ca}(\text { gat })_{2}\left(\mathrm{H}_{2} \mathrm{O}\right)_{2}\right] \mathrm{Cl}_{2} \cdot 2 \mathrm{H}_{2} \mathrm{O}$} & antifungal activity & \\
\hline & $\mathrm{Cr}^{3+}$ & & {$\left[\mathrm{Cr}(\text { gat })_{2} \mathrm{Cl}\left(\mathrm{H}_{2} \mathrm{O}\right)_{2}\right] \mathrm{Cl} \cdot 2 \mathrm{H}_{2} \mathrm{O}$} & antiiinflamatory & \\
\hline & $\mathrm{Mn}^{2+}$ & & {$\left[\mathrm{Mn}(\text { gat })_{2}\left(\mathrm{H}_{2} \mathrm{O}\right)_{2}\right] \cdot 6 \mathrm{H}_{2} \mathrm{O}$} & & \\
\hline & $\mathrm{Fe}^{3+}$ & & {$\left[\mathrm{Fe}(\mathrm{gat})_{2} \mathrm{Cl}\left(\mathrm{H}_{2} \mathrm{O}\right)_{2}\right] \mathrm{Cl} \cdot 2 \mathrm{H}_{2} \mathrm{O}$} & & \\
\hline & $\mathrm{Co}^{2+}$ & & {$\left[\mathrm{Co}(\text { gat })_{2}\left(\mathrm{H}_{2} \mathrm{O}\right)_{2}\right] \cdot 4 \mathrm{H}_{2} \mathrm{O}$} & & \\
\hline & $\mathrm{Ni}^{2+}$ & & {$\left[\mathrm{Ni}\left(\mathrm{gat}_{2}\left(\mathrm{H}_{2} \mathrm{O}\right)_{2}\right] \mathrm{Cl}_{2} \cdot 2 \mathrm{H}_{2} \mathrm{O}\right.$} & & \\
\hline & $\mathrm{Cu}^{2+}$ & & {$\left[\mathrm{Cu}(\text { gat })_{2}\left(\mathrm{H}_{2} \mathrm{O}\right)_{2}\right] \cdot \mathrm{H}_{2} \mathrm{O}$} & & \\
\hline & $\mathrm{Zn}^{2+}$ & & {$\left[\mathrm{Zn}(\text { gat })_{2}\left(\mathrm{H}_{2} \mathrm{O}\right)_{2}\right] \cdot 2 \mathrm{H}_{2} \mathrm{O}$} & & \\
\hline & $\mathrm{Cd}^{2+}$ & & {$\left[\mathrm{Cd}(\mathrm{gat})_{2}\left(\mathrm{H}_{2} \mathrm{O}\right)_{2}\right] \mathrm{Cl}_{2} \cdot 4 \mathrm{H}_{2} \mathrm{O}$} & & \\
\hline
\end{tabular}


Table 6. Cont.

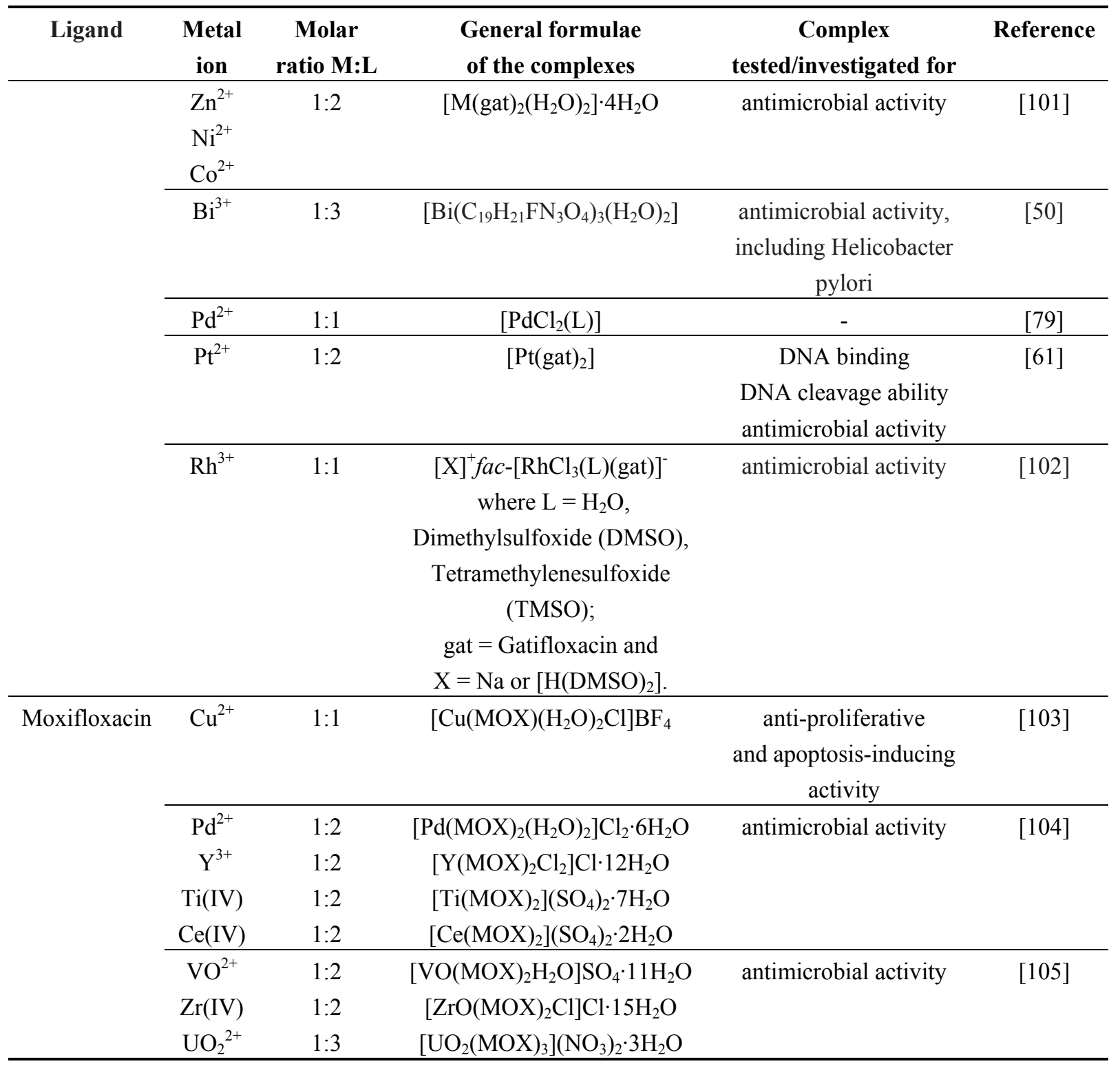

The first review regarding the interactions of metal ions with quinolone was published ten years ago and discussed selected crystal structures of quinolone-metal compounds, different physico-chemical methods of characterization, as well as some results of bioactivity test [21]. The structural characteristics of a part of fluoroquinolone complexes and their biological activity were reviwed four years ago [106]. A recent comprehensive review [107] presented the structures and the biological activity of complexes of some quinolones with $\mathrm{Cu}(\mathrm{II}), \mathrm{Ni}(\mathrm{II}), \mathrm{Co}(\mathrm{II})$ and $\mathrm{Zn}$ (II) and analysed the influence of the second ligand on biological activity.

In one report, norfloxacin acts as bidentate ligand through two carboxylate oxygen atoms (Figure 6) in complexes with $\mathrm{Co}(\mathrm{II})$ and $\mathrm{Fe}$ (III) ions [108]. A quite rare coordination mode of quinolones occurs in a bidentate fashion via the piperazine nitrogen atoms. This coordination was reported in complexes of general formula $\left[\mathrm{PtCl}_{2}(\mathrm{~L})\right]$ (Figure 7) formed by ciprofloxacin, levofloxacin, ofloxacin, sparfloxacin, and gatifloxacin with Pt(II) [109], and could be explained through the basicity both of N4 nitrogen from piperazine ring and of N1 nitrogen, the last one evidenced in recent studies [110]. 
Figure 6. The proposed structure of complexes of Fe(III)-Nf and Co(II)-Nf (adapted from [108]).<smiles>CCCCCn1cc(C(=O)OC(=O)c2cn(CC)c3cc(N4CCNCC4)c(F)cc3c2=O)c(=O)c2cc(F)c(N3CCNCC3)cc21</smiles>

Figure 7. Proposed structure for $\left[\mathrm{PdCl}_{2}(\mathrm{~L})\right]$ (adapted from [104]).<smiles>O=C(O)c1cn(C2CC2)c2cc(N3CCN(Cl)CC3)c(F)cc2c1=O</smiles>

\subsection{Chelates Introduced into the Polyoxometalates (POMs) Surface}

Quinolone molecules are excellent multidentate ligands able to construct metal-organic polymers with medical applications, due to the higher electronic cloud density of oxygen and nitrogen atom [111]. Such hybrid organic-inorganic materials have been obtained by introducing a quinolone chelate into the surface of a polyoxometalate anion. The polyoxometalates (POMs) are known as anti-tumor, antiviral, and antibacterial inorganic medical agents, and the modifying of their surface with such compounds with biological activity is aimed to ameliorate their properties.

Generally, these complexes were obtained by hydrothermal reaction of a quinolone with a metal salt and a polyoxometalate (in the acidic form or as ammonium salt) with adjusting the $\mathrm{pH}$.

One of the simplest compound of this series is $\left.\mathrm{V}_{4} \mathrm{O}_{10}\left(\mu_{2}-\mathrm{O}\right)_{2}\left[\mathrm{VO}(\mathrm{H}-\mathrm{Cf})_{2}\right)\right]_{2} \cdot 13 \mathrm{H}_{2} \mathrm{O}$, with a structure consisting in one $\left\{\mathrm{V}_{4} \mathrm{O}_{12}\right\}$ unit and two corner-sharing octahedral $\left\{\mathrm{VO}_{6}\right\}$-ciprofloxacin units linked through two $\mu_{2}-\mathrm{O}$ bridges [112].

Anions with $\alpha$-Keggin structure $\left(\mathrm{PW}_{12} \mathrm{O}_{40}{ }^{4-}, \mathrm{SiW}_{12} \mathrm{O}_{40}{ }^{4-}\right)$ were used as inorganic building blocks in compounds constructed from $\mathrm{PW}_{12}$ or $\mathrm{SiW}_{12}$ clusters and two $\mathrm{M}(\mathrm{Quin})_{2}$ chelates. The $\mathrm{PW}_{12}$ or $\mathrm{SiW}_{12}$ clusters and quinolone molecule as chelating bidentate organic ligands coordinate the metal ions together (Figure 8). The binuclear metal clusters are connected to the POM clusters, bound as unidentate or as bridging bi-dentate inorganic ligands, forming a 1D chain architecture, as shown in Figure 9. 
Figure 8. A binuclear metallic cluster of quinolone bound to POM clusters.

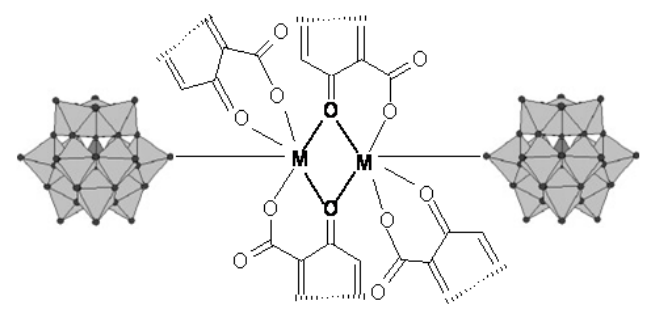

Figure 9. Schematic representation of the 1D chain structure, constructed by POMs and M-quin binuclear clusters with POM bound as (a) bidentate bridging ligand or (b) unidentate ligand.

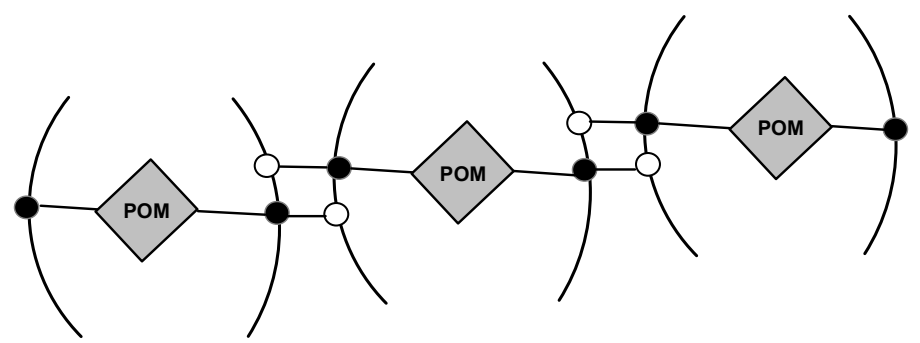

(a)

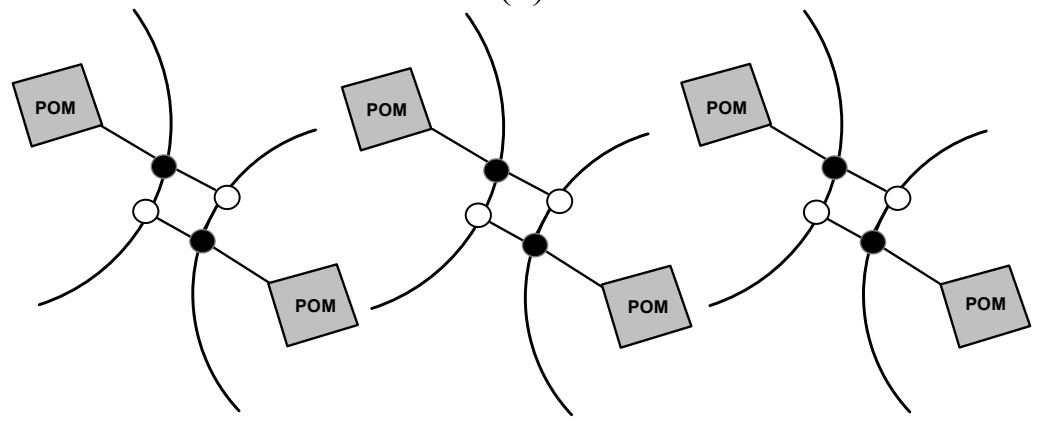

(b)

Starting to polyoxometalates (POMs) and the quinolone antibacterial drug pipemidic acid (HPPA), complexes as $\left\{\left[\mathrm{Co}(\mathrm{PPA})_{2}\right] \mathrm{H}_{2}\left[\mathrm{SiW}_{12} \mathrm{O}_{40}\right]\right\} \cdot \mathrm{HPP} \cdot 3 \mathrm{H}_{2} \mathrm{O} \quad[113], \quad\left[\mathrm{Cu}(\mathrm{PPA})_{2}\right]_{2} \cdot\left[\mathrm{PW}_{12} \mathrm{O}_{40}\right] \cdot 6 \mathrm{H}_{2} \mathrm{O} \quad[114]$, $\left\{\left[\mathrm{Ni}(\mathrm{PPA})_{2}\right] \mathrm{H}_{4}\left[\mathrm{SiW}_{12} \mathrm{O}_{40}\right]\right\} \cdot \mathrm{HPPA} \cdot 3 \mathrm{H} 2 \mathrm{O}$, and $\left\{\left[\mathrm{Zn}(\mathrm{PPA})_{2}\right]_{2} \mathrm{H}_{4}\left[\mathrm{SiW}_{12} \mathrm{O}_{40}\right]\right\} \cdot 3 \mathrm{H}_{2} \mathrm{O}$ [115] were obtained. By introducing different quinolone antibacterial drugs into the octamolybdate POMs new compounds have been isolated, such as $\left[\mathrm{Cu}^{\mathrm{II}}\left(\mathrm{L}^{1}\right)_{2}\left(\mathrm{H}_{2} \mathrm{O}\right)_{2}\right] \mathrm{H}_{2}\left[\beta-\mathrm{Mo}_{8} \mathrm{O}_{26}\right] \cdot 4 \mathrm{H}_{2} \mathrm{O}(1),\left[\mathrm{Cu}_{2}{ }_{2}{ }^{\mathrm{II}}\left(\mathrm{L}^{2}\right)_{4}\right]\left[\delta-\mathrm{Mo}_{8} \mathrm{O}_{26}\right] \cdot 4 \mathrm{H}_{2} \mathrm{O}$ (2), $\left[\mathrm{Cu}_{2}{ }_{2}\left(\mathrm{~L}^{3}\right)_{2}\left(\mathrm{H}_{2} \mathrm{O}\right)_{2}\right]\left[\beta-\mathrm{Mo}_{8} \mathrm{O}_{26}\right](3),\left[\mathrm{Cu}_{2}{ }_{2}\left(\mathrm{~L}^{4}\right)_{2}\left(\mathrm{H}_{2} \mathrm{O}\right)_{4}\right]\left[\beta-\mathrm{Mo}_{8} \mathrm{O}_{26}\right] \cdot 2 \mathrm{H}_{2} \mathrm{O}$ (4) (where $\mathrm{L}^{1}=$ enrofloxacin; $\mathrm{L}^{2}=$ pipemidic acid; $\mathrm{L}^{3}=$ norfloxacin; $\mathrm{L}^{4}=$ enoxacin) [111].

\subsection{Metal Complexes with Quinolone Acting as Unidentate Ligand}

The quinolones bearing a piperazinyl ring in the 7-position could form complexes where the terminal piperazinyl nitrogen (N4) is involved in the coordination to the metal ion. This coordination mode was reported for complexes with transition metals $\mathrm{Ag}(\mathrm{I}), \mathrm{Au}(\mathrm{III})$, and $\mathrm{Ru}(\mathrm{III})$. The structure proposed for the complex $\mathrm{Ag}_{2}(\mathrm{Nf})_{2}\left(\mathrm{NO}_{3}\right)_{2}[116]$ is presented in Figure 10. 
Figure 10. Proposed structure for the complex $\mathrm{Ag}(\mathrm{H}-\mathrm{Nf})_{2}\left(\mathrm{NO}_{3}\right)[116]$.<smiles></smiles>

By the reaction of $\mathrm{Ag}(\mathrm{I})$ and $\mathrm{Au}(\mathrm{III})$ with norfloxacin, a dinuclear complex $\mathrm{Ag}_{2}\left(\mathrm{Nf}_{2}\left(\mathrm{NO}_{3}\right)_{2}\right.$ [Figure 11(a)], and a mononuclear complex $\left[\mathrm{Au}(\mathrm{Nf})_{2}\left(\mathrm{H}_{2} \mathrm{O}\right)_{2}\right] \mathrm{Cl}_{3}$ [Figure 11(b)] were obtained [117].

Figure 11. Proposed structures for (a) $\mathrm{Ag}_{2}(\mathrm{Nf})_{2}\left(\mathrm{NO}_{3}\right)_{2}$, and (b) $\left[\mathrm{Au}(\mathrm{Nf})_{2}\left(\mathrm{H}_{2} \mathrm{O}\right)_{2}\right] \mathrm{Cl}_{3}[117]$.

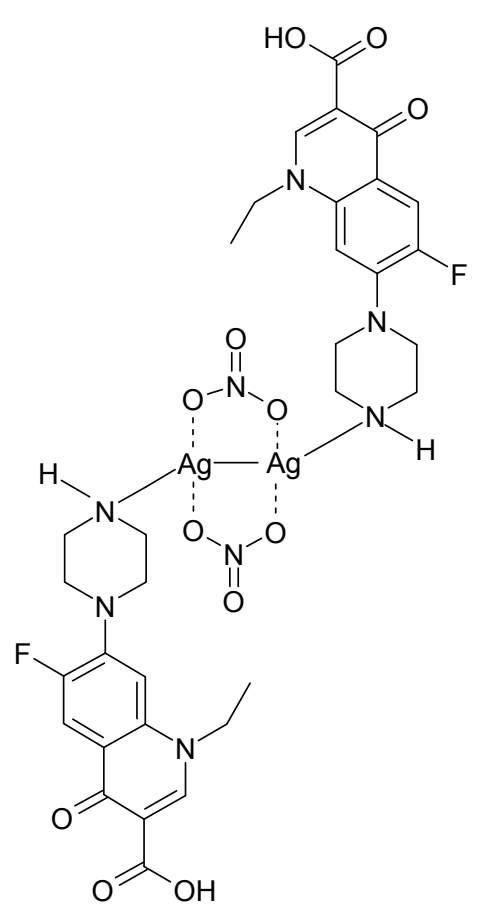

(a)<smiles>CCn1cc(C(=O)O)c(=O)c2cc(F)c(N3CCN([Al](O)O)CC3)cc21</smiles>

(b)

In some complexes of $\mathrm{Ru}(\mathrm{III})$, formulated as $\mathrm{Ru}(\mathrm{L})_{2} \mathrm{Cl}_{3}(\mathrm{DMSO})_{\mathrm{m}} \cdot \mathrm{xH}_{2} \mathrm{O}$ (L: pipemidic acid, enoxacin, enrofloxacin, ciprofloxacin, norfloxacin, ofloxacin, levofloxacin), quinolones are bound as unidentate ligand coordinate through the N4 piperazinyl nitrogen [118,119].

\subsection{Polymeric Complexes}

Dimeric complexes $\left[\mathrm{Mg}_{2}\left(\mathrm{H}_{2} \mathrm{O}\right)_{6}(\mathrm{HNf})_{2}\right] \mathrm{Cl}_{4} \cdot 4 \mathrm{H}_{2} \mathrm{O}$ and $\left[\mathrm{Ca}_{2}(\mathrm{Cl})(\mathrm{HNf})_{6}\right] \mathrm{Cl}_{3} \cdot 10 \mathrm{H}_{2} \mathrm{O}$ [120] are formed with norfloxacin as bidentate bridging ligand bound through the pyridone oxygen and one carboxylate oxygen atom (unidentate bridging) (Figure 12). 
Figure 12. Structure of the dimeric complex $\left[\mathrm{Mg}_{2}\left(\mathrm{H}_{2} \mathrm{O}\right)_{6}(\mathrm{HNf})_{2}\right] \mathrm{Cl}_{4} \cdot 4 \mathrm{H}_{2} \mathrm{O}$ (adapted from [120]).

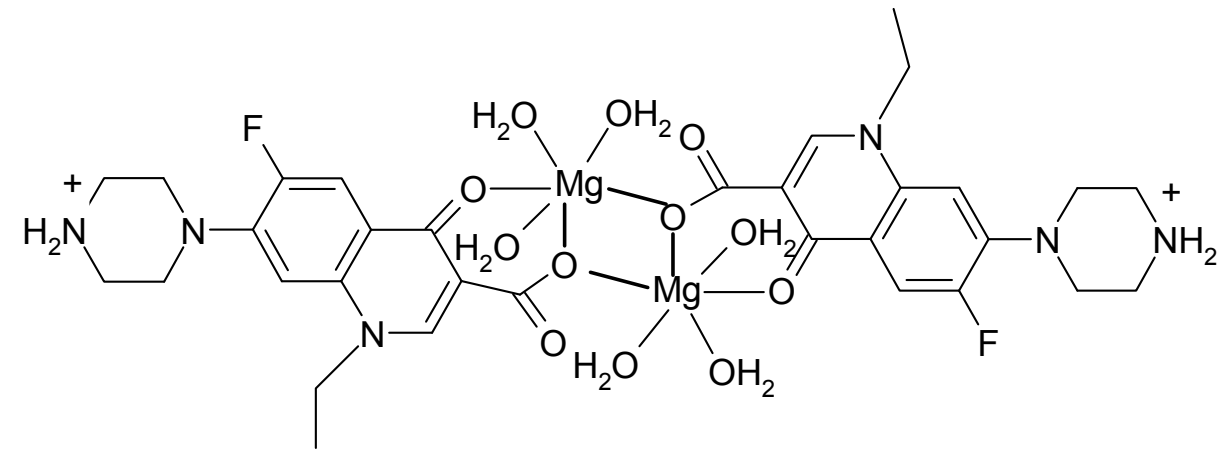

A similar coordination it was found in the complex $\left[\mathrm{Pb}(\mathrm{H}-\mathrm{Nf})\left(\mathrm{ONO}_{2}\right)_{2}\right]_{2}$ (Figure 13) [121].

Figure 13. Structure of the dimeric complex $\left[\mathrm{Pb}(\mathrm{H}-\mathrm{Nf})\left(\mathrm{ONO}_{2}\right)_{2}\right]_{2}$ (adapted from [121]).

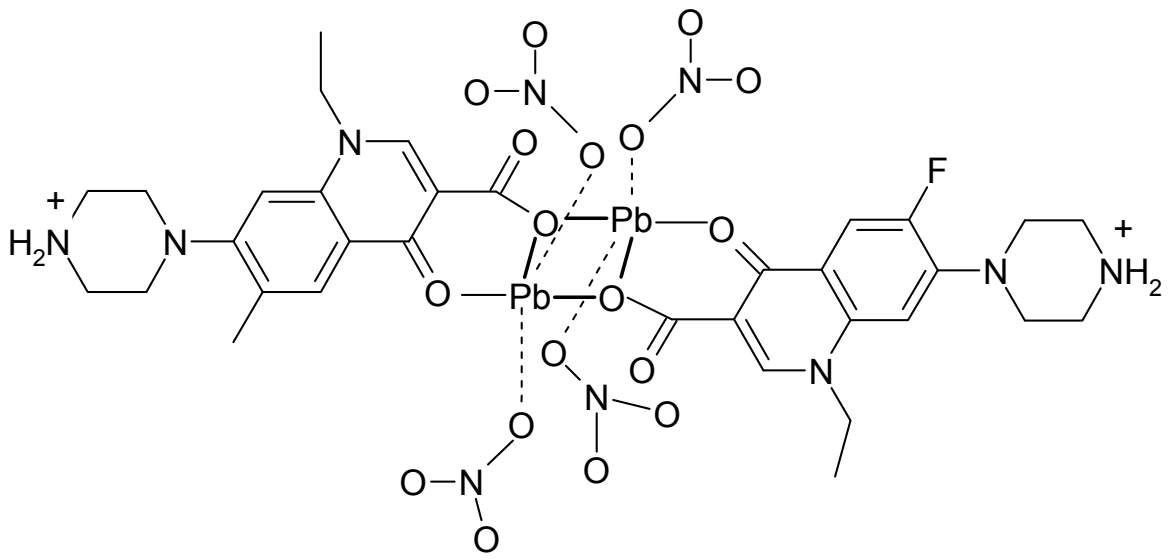

X-ray determination of crystal structure of the dinuclear complexes $\left[\mathrm{Cd}_{2}(\mathrm{Cx})_{4}\left(\mathrm{H}_{2} \mathrm{O}\right)_{2}\right] \cdot 10 \mathrm{H}_{2} \mathrm{O}$ and $\left[\mathrm{Cd}_{2}(\mathrm{Cx})_{4}(\mathrm{DMSO})_{2}\right] \cdot 2 \mathrm{H}_{2} \mathrm{O}$ revealed that the cadmium ion is heptacoordinated; the coordination environment consists in two cinoxacinate ions acting as tridentate chelate and bridging ligands, one as bidentate chelate ligand, and one water molecule [33].

In polymeric complexes, different modes of coordination are simultaneously possible. In the case of two Fe(II) complexes, norfloxacin adopts different modes of coordination depending on the synthesis conditions. In the structure of $\mathrm{Fe}(\mathrm{H}-\mathrm{Nf})_{2}\left(\mathrm{SO}_{4}\right) \cdot 2 \mathrm{H}_{2} \mathrm{O}, \mathrm{Fe}(\mathrm{II})$ is surrounded by two norfloxacinate anions bound as bidentate ligand coordinated through the pyridone oxygen and one carboxyl carboxylate oxygen and two norfloxacin molecules coordinated as unidentate ligand by two oxygen atoms from two different carboxylate [Figure 14(a)]. In the other complex, $\mathrm{Fe}(\mathrm{Nf})_{2} \cdot 4 \mathrm{H}_{2} \mathrm{O}$, two molecules are bound as bidentate ligand, and two as unidentate ligand coordinated through piperazine nitrogen [Figure 14(b)] [122]. 
Figure 14. Coordination modes of norfloxacin in (a) $\mathrm{Fe}(\mathrm{H}-\mathrm{Nf})_{2}\left(\mathrm{SO}_{4}\right) \cdot 2 \mathrm{H}_{2} \mathrm{O}$ and (b) $\mathrm{Fe}(\mathrm{Nf})_{2} \cdot 4 \mathrm{H}_{2} \mathrm{O}$ (adapted from [122]).
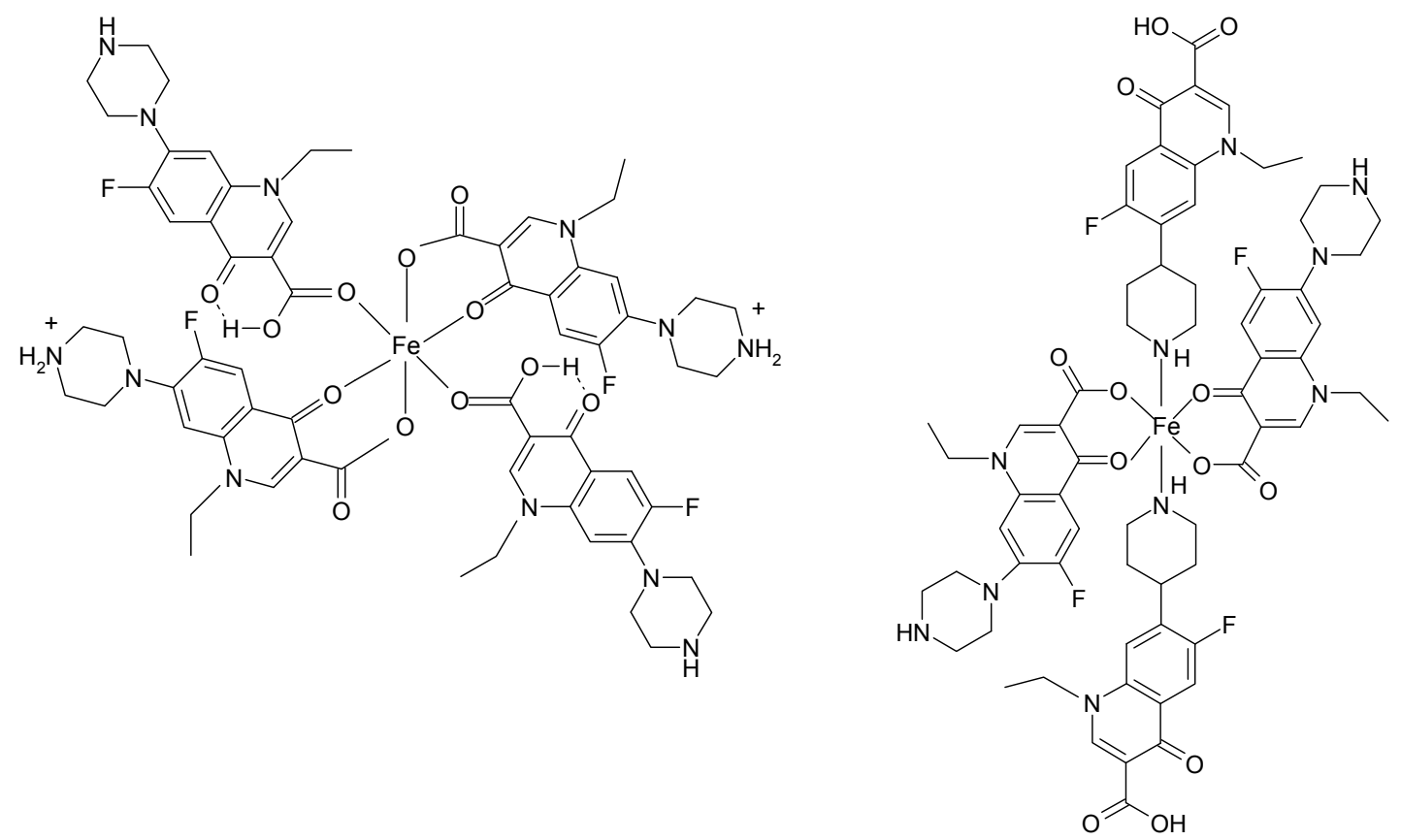

In a $1 \mathrm{D}$ ladder-like silver(I) coordination polymer, $\left\{\left[\mathrm{Ag}_{4}(\mathrm{H}-\mathrm{Cf})_{2}(\mathrm{Cf})_{2}\left(\mathrm{NO}_{3}\right)_{2}\right] \cdot 4 \mathrm{H}_{2} \mathrm{O}\right\}_{\mathrm{n}}$ [123] the pseudo-tetranuclear building blocks are constructed via unidentate ciprofloxacin coordinated through the N4 piperazine atom and tetradentate deprotonated ciprofloxacin ligands (Figure 15).

Figure 15. Coordination modes of ciprofloxacin and its anion in $\left\{\left[\mathrm{Ag}_{4}(\mathrm{H}-\mathrm{Cf})_{2}(\mathrm{Cf})_{2}\left(\mathrm{NO}_{3}\right)_{2}\right] \cdot 4 \mathrm{H}_{2} \mathrm{O}\right\}_{\mathrm{n}}[123]$.<smiles>O=C(O)c1cn(C2CC2)c2cc(N3CCNCC3)c(F)cc2c1=O</smiles>

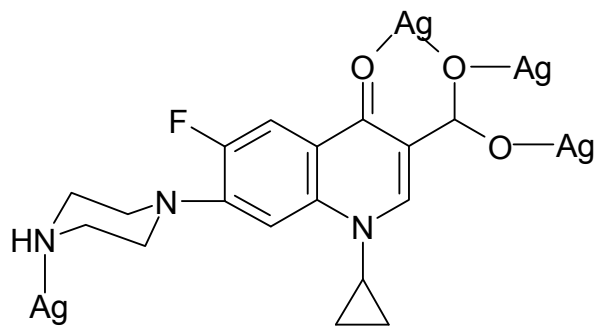

\subsection{Ionic Complexes}

Based on the basic function of the N4 pyperazinyl atom, quinolones are protonated in acidic medium, forming ionic chlometalates, generally obtained by slow evaporation of an acidic solution of complex and metal salt. Most of these complexes were tested for their antimicrobial activity (see Subsection 4.3).

The chloroantimonates (III) obtained with nalidixium $\mathrm{C}_{12} \mathrm{H}_{13} \mathrm{~N}_{2}$ (nalidixium cation) and ciprofloxacinium ions have the general formulae $\left(\mathrm{C}_{12} \mathrm{H}_{13} \mathrm{~N}_{2} \mathrm{O}_{3}\right)\left[\mathrm{SbCl}_{4}\right] \cdot \mathrm{H}_{2} \mathrm{O}$ [124], and $\left(\mathrm{C}_{17} \mathrm{H}_{19} \mathrm{~N}_{3} \mathrm{O}_{3} \mathrm{~F}\right)$ $\left[\mathrm{SbCl}_{5}\right] \cdot \mathrm{H}_{2} \mathrm{O}$ (ciprofloxacinium cations $\left.\left(\mathrm{CfH}_{3}\right)^{2+}\right)$ [125] respectively. Two types of chlorobismutates (III) were obtained with ciprofloxacin, $\left(\mathrm{CfH}_{2}\right)(\mathrm{CfH})\left[\mathrm{BiCl}_{6}\right] \cdot 2 \mathrm{H}_{2} \mathrm{O}[126]$ and $\left(\mathrm{CfH}_{2}\right)_{2}\left[\mathrm{Bi}_{2} \mathrm{Cl}_{10}\right] \cdot 4 \mathrm{H}_{2} \mathrm{O}[127]$. 
The tetrachlorocuprates (II) synthesized from norfloxacin, pefloxacin, and cinoxacin, were formulated as $\left(\mathrm{NfH}_{2}\right)(\mathrm{NfH})\left[\mathrm{CuCl}_{4}\right] \mathrm{Cl} \cdot \mathrm{H}_{2} \mathrm{O}$ [128], $\left(\mathrm{C}_{17} \mathrm{H}_{22} \mathrm{FN}_{3} \mathrm{O}_{3}\right)^{2+}\left[\mathrm{CuCl}_{4}\right]^{2-} \quad$ [129], and $\left(\mathrm{CxH}_{2}\right)\left[\mathrm{CuCl}_{4}\right] \cdot \mathrm{H}_{2} \mathrm{O}[129]$, respectively.

Other chloromethalates, such as enrofloxacinium tetrachloroferate (II), $\left(\mathrm{erxH}_{2}\right)\left[\mathrm{FeCl}_{4}\right] \mathrm{Cl}[130]$, ciprofloxacinium tetrachlorozincate (II) dihydrate, $\left[\mathrm{C}_{17} \mathrm{H}_{19} \mathrm{~N}_{3} \mathrm{O}_{3} \mathrm{~F}\right]_{2}\left[\mathrm{ZnCl}_{4}\right] \cdot 2 \mathrm{H}_{2} \mathrm{O} \quad[131]$, ciprofloxacinium tetrachloroaurate (III) monohydrate, $\left(\mathrm{CfH}_{2}\right)\left[\mathrm{AuCl}_{4}\right] \cdot \mathrm{H}_{2} \mathrm{O}$ [132] and ciprofloxacinium hexachlororuthenate (III) trihydrate, $\left(\mathrm{CfH}_{2}{ }^{+}\right)_{3}\left[\mathrm{RuCl}_{6}\right] \cdot 3 \mathrm{H}_{2} \mathrm{O}[78]$ were also reported.

\section{Consequences and Applications of Metal-Quinolone Complexation}

\subsection{Pharmaceutical Aspects}

Some chelates of quinolones with trivalent cations have shown an improved solubility compared to that of the free ligand, and this behaviour could be advantageous for pharmaceutical formulation. The hydrochlorides of the aluminium (III) complexes of ciprofloxacin and norfloxacin were reported $[48,133]$. Both complexes are more soluble than the antibiotics themselves. The complexes can be used for developing more dose-efficient formulations, such as compressed tablet dosage forms [48,134]. The pharmacodynamic properties of ciprofloxacin are not drastically affected upon complexation with aluminium. The complex $\left[(\mathrm{HCl} \cdot \mathrm{Cf})_{3} \mathrm{Al}\right]$ showed a longer post-antibiotic effect (PAE) compared to that the free ciprofloxacin [135].

The solubility studies of a bismuth (III) complex of norfloxacin, $\left[\mathrm{Bi}\left(\mathrm{C}_{16} \mathrm{H}_{18} \mathrm{FN}_{3} \mathrm{O}_{3}\right)_{4}\left(\mathrm{H}_{2} \mathrm{O}\right)_{2}\right](\mathrm{BNC})$ in different $\mathrm{pH}$ buffers indicated that the solubility of the $\mathrm{BNC}$ was higher than that of norfloxacin until pH 6.5. Above this $\mathrm{pH}$ value, a significant decrease in the solubility of BNC was observed, while the solubility of norfloxacin did not change significantly. The increased solubility can be an advantage for the antibacterial activity of the bismuth complex [49].

\subsection{Biopharmaceutical and Pharmacokinetic Implications}

Reducing the oral bioavailability of quinolones in the presence of multivalent cations is the main consequence of the metal ions-quinolones interaction, and it was reported for the first time in 1985 [136]. A reduction in ciprofloxacin biavailability in healthy human subjects was observed at co-administration with ferrous salts and a combination of multi-vitamin and mineral preparation. In correlation with UV-Vis spectra features, the formation of a 1:3 ferric ion-ciprofloxain complex was proposed as the cause of the reduction in ciprofloxacin biovailability [137]. A strong correlation between the reduction in oral bioavailability of norfloxacin in the presence of divalent and trivalent cations and the magnitude of formation constants measured in vitro was established $\left(\mathrm{Ca}^{2+}<\mathrm{Mg}^{2+}<\mathrm{Zn}^{2+} \sim \mathrm{Fe}^{2+}<\right.$ $\mathrm{Al}^{3+}$ ). A marked difference between the effect of $\mathrm{Zn}^{2+}$ and $\mathrm{Fe}^{2+}$ was observed in vivo, namely a greater reduction in norfloxacin absorption with co-administration of $\mathrm{Fe}^{2+}$. The oxidation of $\mathrm{Fe}^{2+}$ to $\mathrm{Fe}^{3+}$ in gastrointestinal tract was proposed as possible explanation [138].

Several mechanisms were proposed in order to explain the decreased biovailability of quinolone in the presence of metal ions. The first hypothesis was that the reduction of quinolone absorption is due to the formation of insoluble and unabsorbable chelates in the gastrointestinal tract [139-141]. On the contrary, in other studies it was observed that the solubility of lomefloxacin increases in the presence 
of $\mathrm{Ca}^{2+}, \mathrm{Mg}^{2+}, \mathrm{Al}^{3+}$ şi $\mathrm{Fe}^{3+}$ ions [142]. This means that the reduction of the gastric absorption of lomefloxacin at co-administration with these metal ions, are not caused by the precipitation, but by a decrease of the octanol-water partition cofficient. Only for $\mathrm{Bi}^{3+}$, solubility and thus absorption of lomefloxacin, decresed as a result of formation of species with low solubility [143]. The permeability through intestinal mucosa of fluoroquinolone alone and in the presence of metal ions was studied in vitro. The effect of $\mathrm{Ca}^{2+}, \mathrm{Mg}^{2+}, \mathrm{Fe}^{2+}$ was tested with ciprofloxacin, while the effect of $\mathrm{Al}^{3+}$ was tested with ciprofloxacin, norfloxacin and ofloxacin. The experimental data revealed that the fluoroquinolone-metal ion combinations resulted in a reduced intestinal permeability compared to that of the corresponding fluoroquinolone, leading to a reduction of fluoroquinolone bioavailability [144].

\subsection{Mechanism of Action of Quinolones}

The DNA-binding capacity of quinolone complexes was studied in relation with the mechanism of action of quinolones. Experimental data suggested an interaction of quinolone- $\mathrm{Mg}^{2+}$ complex with DNA and gyrase and not a direct interaction of free quinolones with DNA, and a model for the ternary complex was proposed. In this model, $\mathrm{Mg}^{2+}$ acts as a bridge between the phosphate groups of the nucleic acid and the carbonyl and carboxyl moieties of norfloxacin, with additional stabilization arising from stacking interactions between the condensed rings of the drug and DNA bases [145].

Interaction of an oligonucleotide duplex and ciprofloxacin in the absence and in the presence of $\mathrm{Mg}^{2+}$ was studied and a model of the ternary $\mathrm{Cf}-\mathrm{Mg}^{2+}$-duplex adduct orientation was proposed. Docking carried out on this model sustained the orientation of the $\mathrm{CFX}-\mathrm{Mg}^{2+}$ in the minor groove of DNA [146].

Interaction with calf thymus DNA was investigated in vitro using different associations between quinolone and divalent metal ions: norfloxacin- $\mathrm{Cu}^{2+}$ [147], ciprofloxacin- $\mathrm{Mg}^{2+},-\mathrm{Cu}^{2+}[148,149]$, levofloxacin- $\mathrm{Cu}^{2+}[150]$, gatifloxacin- $\mathrm{Mg}^{2+},-\mathrm{Cu}^{2+}[149,151],-\mathrm{Co}^{2+},-\mathrm{Cd}^{2+}$ [151], fleroxacin- $\mathrm{Mg}^{2+}$, $-\mathrm{Cu}^{2+}$ [146], sparfloxacin- $\mathrm{Mg}^{2+}$ [149,152], $-\mathrm{Cu}^{2+}$ [149], - $\mathrm{Cd}^{2+}$ [152], -Cr(III), -Cr(VI) [153], pazufloxacin- $\mathrm{Cu}^{2+}[154]$.

From the experimental results, it was concluded that the metal ion plays an intermediary role in the interaction between quinolone and DNA, and the metal complex of quinolone can interact with DNA by an intercalative binding model $[155,156]$. In vitro experiments demonstrated the hypothesis that, on the one hand, DNA gyrase cannot bind quinolones in the absence of DNA, and on the other hand, the quinolone-gyrase-DNA complex is formed in the presence of $\mathrm{Mg}^{2+}$.

Magnesium and related metal ions affect the stability and function of topoisomerases: they reduce the stability of protein thus increasing the structural flexibility required for the structural changes involved in catalytic cycle [157,158]. On the other side, the divalent metal ions (especially $\mathrm{Mg}^{2+}$ ) might play a role in enzyme poisoning due to their ability to bind the topoisomerase II-directed drugs, including quinolones [158]. The coordination environment proposed for $\mathrm{Mg}^{2+}$ bound to topoisomerase IV consists in two C3/C4 oxygen atoms from a quinolone molecule chelated and four water molecules. Two of these water molecules are involved in hydrogen bonds with serine side chain hydroxyl group and with serine glutamic acid side chain carboxyl group. It was suggested that the interaction between quinolone and topoisomerases is mediated by this water-metal ion "bridge" [159]. Mutations of one of 
both amino acid residues that disrupt the bridge function partially or total, and thus the proteinquinolone interaction, are the most common causes of quinolone resistance [160].

\subsection{Metal Complexes with Biological Activity}

\subsubsection{Antimicrobial Activity}

The consequence of interaction with metal ions on the biological activity of quinolones was approached in the first instance as a negative phenomenon, and some evidences of reduction in the antimicrobial activity of quinolones in the presence of metal ions [161,162] support this assumption. Two possible mechanisms were proposed for explaining the reduction of ciprofloxacin activity by metal cations. First of these, especially valid for chelates with 1:1 stoichiometry, could be a decreased permeation of the antibiotic into bacterial cells, while the second one is the formation of an inactive chelate [25].

However, for many chelates of quinolones obtained in solid state, an equal or superior activity was observed compared to that of parent drugs. Selected results expressed as minimal inhibitory concentration (MIC, $\mu \mathrm{g} \mathrm{mL}^{-1}$ ) or as the inhibition diameter zone $(\mathrm{mm})$ are presented in Tables 7 and 8 . Increased biological activity of metal chelates was explained by the overtone concept of cell permeability and chelation theory. Upon chelation, the polarity of a metal ion is reduced due to the partial sharing of positive charge with the donor groups of ligand and as a consequence of overlap with the ligand orbitals. Chelation increases the delocalization of $\pi$ electrons over the whole chelate ring and thus increases the lipophilic nature of the central ion. This increased in lipophilicity enhances the passage of complex through the lipid membranes and the penetration in cells [163-165].

Table 7. Minimal inhibitory concentration ( $\mathrm{MIC}, \mu \mathrm{g} \mathrm{mL}^{-1}$ ) of the drugs for some assayed bacteria.

\begin{tabular}{|c|c|c|c|c|c|c|c|c|}
\hline \multirow{3}{*}{ Compound } & \multicolumn{7}{|c|}{ Bacterial strain } & \multirow{3}{*}{ Ref } \\
\hline & \multicolumn{3}{|c|}{$\operatorname{Gram}(+)$} & \multicolumn{4}{|c|}{ Gram (-) } & \\
\hline & $\begin{array}{c}S . \\
\text { aureus }\end{array}$ & $\begin{array}{c}\text { B. } \\
\text { subtilis }\end{array}$ & $\begin{array}{c}E . \\
\text { faecalis }\end{array}$ & $\begin{array}{c}E . \\
\text { coli }\end{array}$ & $\begin{array}{c}P . \\
\text { aeruginosa }\end{array}$ & $\begin{array}{c}\text { K. } \\
\text { pneumoniae }\end{array}$ & $\begin{array}{c}S . \\
\text { typhimurium }\end{array}$ & \\
\hline Pipemidic acid & 16.0 & - & - & 64.0 & 64.0 & - & - & {$[29]$} \\
\hline$\left[\mathrm{Cu}(\mathrm{PPA})_{2}\left(\mathrm{H}_{2} \mathrm{O}\right)\right]$ & 16.0 & - & - & 8.0 & 8.0 & - & - & \\
\hline$\left[\mathrm{VO}(\mathrm{PPA})_{2}\left(\mathrm{H}_{2} \mathrm{O}\right)\right]$ & 16.0 & - & - & 64.0 & 64.0 & - & - & {$[28]$} \\
\hline$\left[\mathrm{Mn}(\mathrm{PPA})_{2}\left(\mathrm{H}_{2} \mathrm{O}\right)_{2}\right]$ & 16.0 & - & - & 64.0 & 64.0 & - & - & \\
\hline$\left[\mathrm{Fe}(\mathrm{PPA})_{3}\right]$ & 32.0 & - & - & 64.0 & 64.0 & - & - & \\
\hline$\left[\mathrm{Co}(\mathrm{PPA})_{2}\left(\mathrm{H}_{2} \mathrm{O}\right)_{2}\right]$ & 32.0 & - & - & 64.0 & 64.0 & - & - & \\
\hline$\left[\mathrm{Ni}(\mathrm{PPA})_{2}\left(\mathrm{H}_{2} \mathrm{O}\right)_{2}\right]$ & 32.0 & - & - & 64.0 & 32.0 & - & - & \\
\hline$\left[\mathrm{Zn}(\mathrm{PPA})_{2}\left(\mathrm{H}_{2} \mathrm{O}\right)_{2}\right]$ & 32.0 & - & - & 32.0 & 32.0 & - & - & \\
\hline$\left[\mathrm{MoO}_{2}(\mathrm{PPA})_{2}\right]$ & 16.0 & - & - & 64.0 & 64.0 & - & - & \\
\hline$\left[\mathrm{Cd}(\mathrm{PPA})_{2}\left(\mathrm{H}_{2} \mathrm{O}\right)_{2}\right]$ & 16.0 & - & - & 64.0 & 64.0 & - & - & \\
\hline$\left[\mathrm{UO}_{2}(\mathrm{PPA})_{2}\right]$ & 8.0 & - & - & 8.0 & 8.0 & - & - & \\
\hline Cinoxacin & $>64$ & - & $>64$ & 4.0 & $>64$ & 8.0 & 4.0 & {$[33]$} \\
\hline$\left[\mathrm{Cu}(\mathrm{Cx})_{2}\right] \cdot 2 \mathrm{H}_{2} \mathrm{O}$ & $>64$ & - & $>64$ & 4.0 & $>64$ & 8.0 & 4.0 & \\
\hline
\end{tabular}


Table 7. Cont.

\begin{tabular}{|c|c|c|c|c|c|c|c|c|}
\hline \multirow{3}{*}{ Compound } & \multicolumn{7}{|c|}{ Bacterial strain } & \multirow{3}{*}{ Ref. } \\
\hline & \multicolumn{3}{|c|}{$\operatorname{Gram}(+)$} & \multicolumn{4}{|c|}{ Gram (-) } & \\
\hline & $\begin{array}{c}S . \\
\text { aureus }\end{array}$ & $\begin{array}{c}\text { B. } \\
\text { subtilis }\end{array}$ & $\begin{array}{c}E . \\
\text { faecalis }\end{array}$ & $\begin{array}{l}E . \\
\text { coli }\end{array}$ & $\begin{array}{c}P . \\
\text { aeruginosa }\end{array}$ & $\begin{array}{c}\text { K. } \\
\text { pneumoniae }\end{array}$ & $\begin{array}{c}S . \\
\text { typhimurium }\end{array}$ & \\
\hline$\left[\mathrm{Co}(\mathrm{Cx})_{3}\right] \mathrm{Na} \cdot 10 \mathrm{H}_{2} \mathrm{O}$ & $>64$ & - & $>64$ & 2.0 & $>64$ & $2.0^{*}$ & 2.0 & \\
\hline $\mathrm{Cu}(\mathrm{Cx})(\mathrm{HCx}) \mathrm{Cl} \cdot 2 \mathrm{H}_{2} \mathrm{O}$ & $>64$ & - & $>64$ & 4.0 & $>64$ & $8.0^{*}$ & 8.0 & \\
\hline$\left[\mathrm{Zn}(\mathrm{Cx})_{2}\right] \cdot 4 \mathrm{H}_{2} \mathrm{O}$ & $>64$ & - & $>64$ & 4.0 & $>64$ & $4.0^{*}$ & 4.0 & \\
\hline $\mathrm{Cd}(\mathrm{Cx}) \mathrm{Cl} \cdot \mathrm{H}_{2} \mathrm{O}$ & $>64$ & - & 64 & 4.0 & $>64$ & $8.0^{*}$ & 8.0 & \\
\hline$\left[\mathrm{Cd}_{2}(\mathrm{Cx})_{4}(\mathrm{DMSO})_{2}\right] \cdot 2 \mathrm{H}_{2} \mathrm{O}$ & $>64$ & - & 64 & 8.0 & $>64$ & $8.0^{*}$ & 8.0 & \\
\hline$\left[\mathrm{Cd}_{2}(\mathrm{Cx})_{4}\left(\mathrm{H}_{2} \mathrm{O}\right)_{2}\right] \cdot 10 \mathrm{H}_{2} \mathrm{O}$ & $>64$ & - & 64 & 4.0 & $>64$ & $4.0^{*}$ & 4.0 & \\
\hline Oxolinic acid & 16 & - & - & 1 & 16 & - & - & {$[35]$} \\
\hline$\left[\mathrm{Cu}(\mathrm{oxo})_{2}\left(\mathrm{H}_{2} \mathrm{O}\right)\right]$ & 64 & - & - & 64 & 32 & - & - & \\
\hline Enoxacin & 1 & 0.25 & 4 & 0.12 & 0.12 & 0.12 & 0.12 & {$[44]$} \\
\hline$\left[\mathrm{Co}(\mathrm{HEx})_{2}\left(\mathrm{ClO}_{4}\right)_{2}\right] \cdot 3 \mathrm{H}_{2} \mathrm{O}$ & 2 & 0.5 & 8 & 0.25 & 0.25 & 0.25 & 0.12 & \\
\hline$\left[\mathrm{Co}(\mathrm{HEx})_{2}\left(\mathrm{NO}_{3}\right)_{2}\right] \cdot 2 \mathrm{H}_{2} \mathrm{O}$ & 1 & 0.25 & 8 & 0.25 & 0.25 & 0.25 & 0.12 & \\
\hline Norfloxacin & 0.060 & - & - & 0.050 & - & 0.075 & - & {$[49]$} \\
\hline$\left[\mathrm{Bi}\left(\mathrm{C}_{16} \mathrm{H}_{18} \mathrm{FN}_{3} \mathrm{O}_{3}\right)_{4}\left(\mathrm{H}_{2} \mathrm{O}\right)_{2}\right]$ & 0.045 & - & - & 0.025 & - & 0.060 & - & \\
\hline Ciprofloxacin & 1 & 0.12 & 1 & 0.03 & 0.5 & 0.03 & 0.016 & {$[44]$} \\
\hline$\left[\mathrm{Cu}(\mathrm{HCf})_{2}\left(\mathrm{NO}_{3}\right)_{2}\right] \cdot 6 \mathrm{H}_{2} \mathrm{O}$ & 0.5 & 0.12 & 0.5 & 0.03 & 1 & 0.06 & 0.03 & \\
\hline$\left[\mathrm{Cu}(\mathrm{HCf})\left(\mathrm{C}_{2} \mathrm{O}_{4}\right)\right] \cdot 2 \mathrm{H}_{2} \mathrm{O}$ & 0.5 & 0.12 & 2 & 0.06 & 1 & 0.06 & 0.06 & \\
\hline Ciprofloxacin & 0.25 & 0.03 & 1 & 0.016 & 0.12 & 0.03 & 0.016 & [34] \\
\hline$\left[\mathrm{Co}(\mathrm{Cf})_{2}\left(\mathrm{H}_{2} \mathrm{O}\right)\right] \cdot 9 \mathrm{H}_{2} \mathrm{O}$ & 0.25 & 0.06 & 1 & 0.004 & 0.12 & 0.016 & 0.008 & \\
\hline$\left[\mathrm{Zn}(\mathrm{Cf})_{2}\left(\mathrm{H}_{2} \mathrm{O}\right)_{2}\right] \cdot 8 \mathrm{H}_{2} \mathrm{O}$ & 0.25 & 0.03 & 1 & 0.004 & 0.12 & 0.03 & 0.016 & \\
\hline $\mathrm{Ni}(\mathrm{Cf})_{2} \cdot 10 \mathrm{H}_{2} \mathrm{O}$ & 0.5 & 0.03 & 1 & 0.12 & 0.12 & 0.03 & 0.016 & \\
\hline $\mathrm{Cu}(\mathrm{Cf})_{2} \cdot 6 \mathrm{H}_{2} \mathrm{O}$ & 0.25 & 0.03 & 1 & 0.004 & 0.12 & 0.03 & 0.008 & \\
\hline Ofloxacin & $0.75 * *$ & 0.5 & 10 & 0.2 & 7 & 0.7 & $0.75 * * *$ & {$[83]$} \\
\hline $\begin{array}{c}{[\mathrm{Mg}(\mathrm{R}-\mathrm{oflo})} \\
\left.(\mathrm{S}-\mathrm{oflo})\left(\mathrm{H}_{2} \mathrm{O}\right)_{2}\right] \cdot 2 \mathrm{H}_{2} \mathrm{O}\end{array}$ & $1 * *$ & 0.8 & 15 & 0.25 & 10 & 1 & $1 * * *$ & \\
\hline Levofloxacin & $0.3 * *$ & 0.3 & 4 & 0.15 & 3 & 0.25 & $0.5 * * *$ & \\
\hline$\left[\mathrm{Mg}(\mathrm{S}-\text { oflo })_{2}\left(\mathrm{H}_{2} \mathrm{O}\right)_{2}\right] \cdot 2 \mathrm{H}_{2} \mathrm{O}$ & $0.6^{* *}$ & 0.5 & 4 & 0.15 & 5 & 0.5 & $0.75 * * *$ & \\
\hline Enrofloxacin & 8 & - & - & 1 & 1 & - & - & {$[93]$} \\
\hline$\left[\mathrm{Cu}(\mathrm{erx})_{2}\left(\mathrm{H}_{2} \mathrm{O}\right)\right.$ & 32 & - & - & 0.125 & 0.125 & - & - & \\
\hline erx & 0.012 & - & - & - & - & - & - & {$[92]$} \\
\hline$\left[\mathrm{Cu}(\mathrm{erx})_{2}\right] \mathrm{Cl}$ & 0.0085 & - & - & - & - & - & - & \\
\hline Herx & 8 & - & - & 1 & 1 & - & - & [89] \\
\hline$\left[\mathrm{VO}(\mathrm{erx})_{2}\left(\mathrm{H}_{2} \mathrm{O}\right)\right]$ & 8 & - & - & 4 & 4 & - & - & \\
\hline$\left[\mathrm{Cu}(\mathrm{erx})_{2}\left(\mathrm{H}_{2} \mathrm{O}\right)\right]$ & 4 & - & - & 0.125 & 0.125 & - & - & \\
\hline$\left[\mathrm{MO}_{2}(\mathrm{erx})_{2}\right]$ & 4 & - & - & 1 & 1 & - & - & \\
\hline
\end{tabular}

Abbreviations: S. aureus, Staphylococcus aureus; B. subtilis, Bacillus subtilis; E. faecalis, Enterococcus (Streptococcus) faecalis; E. coli, Escherichia coli; P. aeruginosa, Pseudomonas aeruginosa; K. Pneumoniae, Klebsiella pneumoniae; S. thyphimurium, Salmonella typhimurium; * Klebsiella spp; **S. epidermidis; ** S. enteriditis. 
Table 8. The inhibition diameter zone values $(\mathrm{mm})$ for norfloxacin and some of its complexes.

\begin{tabular}{ccccc}
\hline \multirow{2}{*}{ Compound } & \multicolumn{3}{c}{ Bacterial strain } & \\
\cline { 2 - 4 } & $\begin{array}{c}\text { Staphylococcus } \\
\text { aureus }\end{array}$ & $\begin{array}{c}\text { Escherichia } \\
\text { coli }\end{array}$ & $\begin{array}{c}\text { Pseudomonas } \\
\text { aeruginosa }\end{array}$ & Reference \\
\hline Norfloxacin & 12 & 25 & 13 & \\
\hline$\left[\mathrm{Y}(\mathrm{NOR})_{2}\left(\mathrm{H}_{2} \mathrm{O}\right)_{2}\right] \mathrm{Cl}_{3} \cdot 10 \mathrm{H}_{2} \mathrm{O}$ & 31 & 39 & 47 & \\
\hline$\left[\mathrm{Pd}(\mathrm{NOR})_{2}\right] \mathrm{Cl}_{2} \cdot 3 \mathrm{H}_{2} \mathrm{O}$ & 27 & 26 & 28 & \\
\hline$\left[\mathrm{La}(\mathrm{nor})_{3}\right] \cdot 3 \mathrm{H}_{2} \mathrm{O}$ & 12 & 10 & 9 & \\
\hline$\left[\mathrm{Ce}(\mathrm{nor})_{3}\right] \cdot 3 \mathrm{H}_{2} \mathrm{O}$ & 12 & 11 & 10 & \\
\hline
\end{tabular}

In fact, many more factors should be considered for metal complexes with antimicrobial activity: (i) the nature of the metal ion; (ii) the nature of the ligands; (iii) the chelate effect; (iv) the total charge of the complex; (iv) the nature of the ion neutralizing the ionic complex; and (vi) the nuclearity of the metal center in the complex $[28,29,89-91,107]$. A detailed comment of the effect of these factors on the biological activity of metal-quinolone complexes was made in a recent review [107].

The results obtained in some particular bacterial strains (Mycobacterium tuberculosis and Helycobacter pylori), which have not been included in Tables 7 and 8, are worth emphasizing distinctively. Fluoroquinolones have been used successfully in helping cure multidrug-resistant tuberculosis, and studies in mice suggest that they can be considered as first line drugs to shorten the duration of therapy [166]. The main drawback with these agents is the high level of resistance, mainly associated with mutation at gyrA or gyrB genes [167,168]. Metal coordination to quinolones can be used not only as strategy to enhance their activity, but also to overcome the drug resistance. The complex of $\mathrm{Cu}(\mathrm{II})$ with ciprofloxacin having general formula $\left[\mathrm{Cu}(\mathrm{Cf})_{2}\left(\mathrm{BF}_{4}\right)_{2}\right] \cdot 6 \mathrm{H}_{2} \mathrm{O}$ exhibited a significant enhancement in the antitubercular activity comparing to ciprofloxacin alone [169]. A series of $\mathrm{Pd}(\mathrm{II})$ and $\mathrm{Pt}(\mathrm{II})$ complexes with general formula $\left[\mathrm{MCl}_{2}(\mathrm{~L})\right]$ (where $\mathrm{L}=$ ciprofloxacin, levofloxacin, ofloxacin, sparfloxacin, and gatifloxacin) were evaluated against Mycobacterium tuberculosis virulent strain H37Rv. The Pd(II) and Pt(II) complexes with sparfloxacin and the $\mathrm{Pt}(\mathrm{II})$ complex with gatifloxacin were the most active within each series in inhibiting bacterial growth, while the least active complexes of the series were the Pd(II) complex with ciprofloxacin and the Pt(II) complex with ofloxacin. Complexes have not shown better antitubercular activity than free gatifloxacin, but their activity was good and, except the complex of $\mathrm{Pd}(\mathrm{II})$ with ciprofloxacin, all of them were more active than rifampicin [79]. The results are in agreement with the in vitro activities of the parent drugs against M. tuberculosis isolated: ciprofloxacin $<$ or $=$ ofloxacin $<$ sparfloxacin $<$ gatifloxacin [170].

Fluoroquinolones from new generations, like levofloxacin, moxifloxacin, gatifloxacin or sitafloxacin have demonstrated efficacy in Helicobacter pylori eradication, in third-line or second-line triple therapy, in combination with a proton pump inhibitor (PPI) and amoxicilin [171,172]. Bismuth-containing quadruple therapy (omeprazole, bismuth, metronidazole and tetracycline) is an alternative first choice treatment for H. pylori [173]. Good results were also obtained with quadruple therapy of bismuth subcytrate-moxifloxacine-tetracycline-lansoprazole (BMTL) with high eradication rate and relatively mild side effects [174]. Starting from these premises, a series of bismuth-fluoroquinolone complexes $\left[\mathrm{Bi}(\mathrm{Flq})_{3}\left(\mathrm{H}_{2} \mathrm{O}\right)_{2}\right]$ (Flq: norfloxacin, ofloxacin, ciprofloxacin, sparfloxacin, lomefloxacin, pefloxacin, 
gatifloxacin) were evaluated for their anti-H. pylori activity, and were found to be more potent against all strains of $H$. pylori used, comparing to the parent FLQs. Moreover, the synthesized complexes also showed high potency against some fluoroquinolone-resistant strains of H. pylori. [50].

\subsubsection{Antifungal and Antiparasitic Activity}

Altough quinolones themselves does not exhibit antifungal activity some of complexes generated by newer fluoroquinolones act not only as antimicrobial agents, but have also shown antifungal activity. Complexes with 1:1 stoichiometry of levofloxacin with $\mathrm{Cr}(\mathrm{III}), \mathrm{Fe}(\mathrm{III}), \mathrm{Co}(\mathrm{II}), \mathrm{Ni}(\mathrm{II}), \mathrm{Cu}(\mathrm{II})$, $\mathrm{Th}(\mathrm{IV}), \mathrm{Mn}(\mathrm{II}), \mathrm{Zn}(\mathrm{II})$ and UO2(II) have proved an antifungal effect higher than the free ligand against Candida albicans [82]. Complexes of gatifloxacin with $\mathrm{Ni}(\mathrm{II}), \mathrm{Cu}(\mathrm{II}), \mathrm{Zn}(\mathrm{II}), \mathrm{Cd}(\mathrm{II}), \mathrm{Fe}(\mathrm{III})$, $\mathrm{Ca}(\mathrm{II}), \mathrm{Mg}(\mathrm{II}), \mathrm{Cr}(\mathrm{III}), \mathrm{Mn}(\mathrm{II})$ and $\mathrm{Co}(\mathrm{II})$ having a stoichiometry 1:2 (metal: ligand) have excellent activity as compared to standard drug toward the fungi Trichophyton rubrum, Candida albicans and Fusarium solani [100].

The complexes $\left[\mathrm{MnCl}_{2}(\mathrm{sf})\left(\mathrm{H}_{2} \mathrm{O}\right)_{2}\right]$ and $\left[\mathrm{CoCl}_{2}(\mathrm{sf})\left(\mathrm{H}_{2} \mathrm{O}\right)_{2}\right]$ displayed a considerable antiparasitic activity against Trypanosoma cruzi. The corresponding complexes of norfloxacin have a differentiated activity: the $\mathrm{Mn}$ (II) complex did not improve the anti-parasitic effect of the free norfloxacin, while the Co(II) complex displayed a 4-fold higher activity than norfloxacin ligand [53].

A new field of research was opened starting to the synthesis of organometallic ruthenium complexes of some quinolone antibacterial agents. The organometallic ruthenium complex of ofloxacin $\left[\left(\eta^{6}-p\right.\right.$-cymene $\left.) \mathrm{RuCl}(\mathrm{O}, \mathrm{O}-\mathrm{oflo})\right] \cdot 2.8 \mathrm{H}_{2} \mathrm{O}$ has a "piano-stool" structure with quinolone acting as bidentate ligand coordinated to the metal through the ring carbonyl and one of the carboxylic oxygen atoms [175]. The complex interacts with DNA and provokes DNA shrinkage. It is moderately active against Trypanosoma brucei rhodesiense, Trypanosoma cruzi and Plasmodium falciparum.

\subsubsection{Anticancer Activity}

The anticancer activity of fluoroquinolones has been explored in the last years [176-179] based on their ability to block topoisomerase II, thus inhibiting the DNA repair activity. It is not surprising that numerous studies concerning the biological activity of quinolone metal complexes include their ability to interact with DNA, as a premise for anticancer activity (see Table 4).

Some complexes of lomefloxacin, $\left[\mathrm{Co}(\mathrm{LFX})\left(\mathrm{H}_{2} \mathrm{O}\right)_{4}\right] \cdot \mathrm{Cl}_{2}$ and $\left[\mathrm{Zn}(\mathrm{LFX})\left(\mathrm{H}_{2} \mathrm{O}\right)_{4}\right] \cdot \mathrm{Cl}_{2}$ were found to be very active against the breast cancer cell line MCF7 [82]. The anti-proliferative activities of the complex $\left[\mathrm{Cu}(\operatorname{mox})\left(\mathrm{H}_{2} \mathrm{O}\right)_{2} \mathrm{Cl}_{3} \mathrm{BF}_{4}\right.$ and of other congeneric complexes with mixed ligands were evaluated against four breast cancer cell lines (MCF-7, T47D, MDA-MB-231 and BT-20), along with the normal breast epithelial MCF-10A cell line, comparing to the parent drug, moxifloxacin. Both the parent ligand as well as its copper complex did not significantly inhibit the proliferation of non-tumorogenic MCF-10A breast epithelial cells. Moxifloxacin did not exhibit anti-proliferative effect against any of the breast cancer cell lines examined, instead, the Co(II) complexes showed differential anti-proliferative activity against the tested breast cancer cell lines [103].

The gold (III) complexes with general formula $\left[\mathrm{AuCl}_{2} \mathrm{~L}\right] \mathrm{Cl}$ ( $\mathrm{L}=$ norfloxacin, levofloxacin, sparfloxacin) were tested against A20 (murine lymphoma), B16-F10 (murine melanoma) and K562 (human myeloid leukemia) tumor cell lines comparing to the normal cell lines L919 (murine lung 
fibroblasts) and MCR-5 (human lung fibroblasts). The free ligands did not showed significant activity in the tumor or normal cell lines, whereas the complexes are more active than the parent drugs, and they have with a similar cytotoxic activity [62].

Recent research has focused on increasing the antitumor activity of polyoxometalates (POMs) by introduction of medicine molecules into the POM surface [180], and such molecules could be quinolone chelates. The first compound obtained by modifying the surface of a POM with a quinolone chelate was the complex $\left\{\left[\mathrm{Co}(\mathrm{PPA})_{2}\right] \mathrm{H}_{2}\left[\mathrm{SiW}_{12} \mathrm{O}_{40}\right]\right\} \cdot \mathrm{HPPA} \cdot 3 \mathrm{H}_{2} \mathrm{O}$. The inhibitory effect against MCF-7 cells lines showed that the complex and pipemidic acid have shown high antitumor activity to MCF-7, whereas the parent compound $\mathrm{SiW}_{12}$ exhibits no antitumor activity to MCF-7. Furthermore, the antitumor activity of complex was higher that that of its parent compounds, and this superiority could be explained from the synergism of POMs and Co-PPA [113]. Other complexes of pipemidic acid, $\left.\left[\mathrm{Cu}(\mathrm{PPA})_{2}\right]_{2} \cdot\left[\mathrm{PW}_{12} \mathrm{O}_{40}\right] \cdot 6 \mathrm{H}_{2} \mathrm{O}\right)$, $[\mathrm{HPPA}]_{5} \cdot\left[\mathrm{PW}_{11} \mathrm{CdO}_{39}\right] \cdot 2 \mathrm{H}_{2} \mathrm{O}$, and $[\mathrm{HPPA}]_{3} \cdot\left[\mathrm{PW}_{12} \mathrm{O}_{40}\right] \cdot 2 \mathrm{H}_{2} \mathrm{O}$ showed a stronger antitumor activity than that of the parent anion against PC-3, Hela and HepG2 cells [114].

It was found that antitumor activity depends on the binding mode of the polyoxoanion. Thus, the complex $\left\{\left[\mathrm{Ni}(\mathrm{PPA})_{2}\right] \mathrm{H}_{4}\left[\mathrm{SiW}_{12} \mathrm{O}_{40}\right]\right\} \cdot \mathrm{HPPA} \cdot 3 \mathrm{H}_{2} \mathrm{O}$, with a $\mathrm{SiW}_{12}$ polyoxoanion acting as a monodentate inorganic ligand covalently linked to the nickel ions, showed no antitumor activity, whereas $\left\{\left[\mathrm{Zn}(\mathrm{PPA})_{2}\right]_{2} \mathrm{H}_{4}\left[\mathrm{SiW}_{12} \mathrm{O}_{40}\right]\right\} \cdot 3 \mathrm{H}_{2} \mathrm{O}$, with a $\mathrm{SiW}_{12}$ polyoxoanion acting as a bi-dentate inorganic ligand covalently linked to the two zinc ions, exhibited higher antitumor activities than its parent compound against MCF-7 lines [115]. The type of polyoxoanion also affects the antitumor activity. This effect was observed for complexes $\left[\mathrm{Cu}^{\mathrm{II}}\left(\mathrm{L}^{1}\right)_{2}\left(\mathrm{H}_{2} \mathrm{O}\right)_{2}\right] \mathrm{H}_{2}\left[\beta-\mathrm{Mo}_{8} \mathrm{O}_{26}\right] \cdot 4 \mathrm{H}_{2} \mathrm{O}(1),\left[\mathrm{Cu}_{2}{ }_{2}\left(\mathrm{~L}^{2}\right)_{4}\right]\left[\delta-\mathrm{Mo}_{8} \mathrm{O}_{26}\right] \cdot 4 \mathrm{H}_{2} \mathrm{O}$ (2), $\left[\mathrm{Cu}_{2} \mathrm{II}_{2}\left(\mathrm{~L}^{3}\right)_{2}\left(\mathrm{H}_{2} \mathrm{O}\right)_{2}\right]\left[\beta-\mathrm{Mo}_{8} \mathrm{O}_{26}\right](3),\left[\mathrm{Cu}_{2}{ }_{2}\left(\mathrm{~L}^{4}\right)_{2}\left(\mathrm{H}_{2} \mathrm{O}\right)_{4}\right]\left[\beta-\mathrm{Mo}_{8} \mathrm{O}_{26}\right] \cdot 2 \mathrm{H}_{2} \mathrm{O}$ (4) (where $\mathrm{L}^{1}=$ enrofloxacin; $\mathrm{L}^{2}=$ pipemidic Acid; $\mathrm{L}^{3}=$ norfloxacin; $\mathrm{L}^{4}=$ enoxacin). The complexes 1,3 , and 4 exhibited a higher effect against SGC7901 lines comparing to the parent compound, while compound 2 showed no anti-SGC7901 activity [111].

The organometallic ruthenium complexes chlorido $\left(\eta^{6}-p\right.$-cymene)(nalidixicato- $\left.\kappa^{2} O, O\right)$ ruthenium(II) and chlorido $\left(\eta^{6}-p\right.$-cymene)(cinoxacinato- $\left.\kappa^{2} O, O\right)$ ruthenium(II) were investigated as anticancer agents in human A549 (nonsmall cell lung carcinoma), CH1 (ovarian carcinoma), and SW480 (colon carcinoma) cells by means of the colorimetric MTT assay and compared to the tumor-inhibiting properties of the respective ligands. Even though the compounds were shown to be mostly non-cytotoxic to the various cell lines, the complexes and all the ligands are inactive in the three cell lines [181].

\subsection{Analytical Applications}

\subsubsection{Determination of Quinolones Based on Complexation with Metal Ions}

The capacity to form complexes with different metal ions has been applied in the analysis of quinolones in pharmaceutical formulations or in biological samples through spectrophotometric, spectroflurimetric and atomic absorption spectrometric methods. Most of the spectrophotometric methods developed for analysis of quinolones are based on the formation of yellow or orange-yellow chelates with $\mathrm{Fe}^{3+}$ in acid medium. The structure of such a complex is depicted in Figure 16. Generally, these methods are simple, rapid, efficient and inexpensive. 
Figure 16. Structure of a 1:2 (metal: ligand) chelate of norfloxacin with $\mathrm{Fe}^{3+}$.

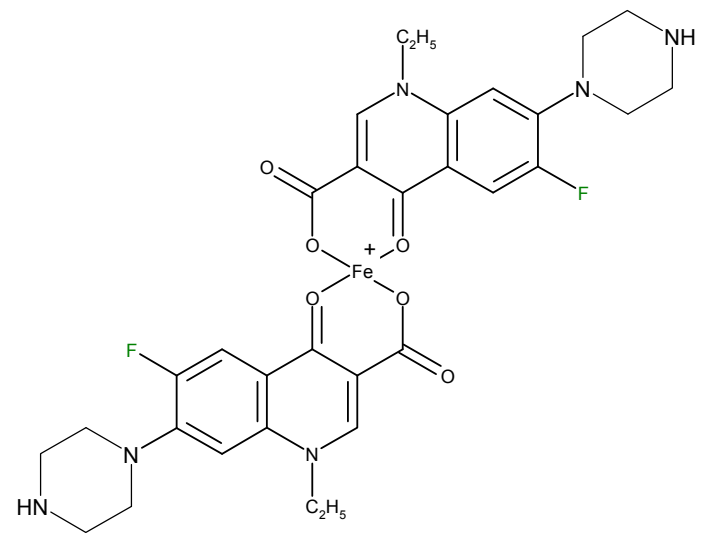

Ciprofloxacin, ofloxacin and norfloxacin have been determined colorimetrically in tablets based on their amber coloured complex with Fe(III) that exhibited a maximum at $370 \mathrm{~nm}$ [182]. The complex with $\mathrm{Fe}^{3+}$ showing a maximum absorption of $435 \mathrm{~nm}$ allowed spectrophotometric determination of ciprofloxacin in tablets and in solution for infusion [183]. Based on the complexation with $\mathrm{Fe}^{3+}$, some flow injection (FI) spectrophotometric methods for determination of norfloxacin in drug formulations were developed. The coloured Fe(III) complexes absorb at $430 \mathrm{~nm}$ [184] or $440 \mathrm{~nm}$ [185]. Ofloxacin has been also determined by a flow-injection spectrophotometric method by measuring the absorbance of its complex with $\mathrm{Fe}^{3+}$ at $420 \mathrm{~nm}$. The method was applied for analysis of ofloxacin in pharmaceuticals and human urine [186]. Ciprofloxacin formed with Fe(III) a brown-red complex whose absorbance was monitored at $447 \mathrm{~nm}$, and the developed method was used for determination of ciprofloxacin in drug formulations [187]. A sequential injection spectrophotometric method was developed for analysis of ciprofloxacin and norfloxacin by measuring the absorbance of the corresponding complexes at $447 \mathrm{~nm}$ and $430 \mathrm{~nm}$, respectively [188].

Chelates with $\mathrm{Fe}(\mathrm{II})$ and $\mathrm{Cu}(\mathrm{II})$ with maximum absorptions placed below $400 \mathrm{~nm}$ were also applied in the spectrophotometric analysis of quinolones. Based on the yellow-coloured chelate with Fe(II) with absorbance at $358 \mathrm{~nm}$, norfloxacin has been determined both in pure form and in tablet form [189]. Norfloxacin, ciprofloxacin and sparfloxacin have been determined in formulations and spiked biological fluids (plasma and urine) via their Cu(II) complexes [190].

Coloured ion-association complexes were applied in developing the new visible spectrophotometric methods for determination of quinolones. Ciprofloxacin and norfloxacin have been determined in pharmaceutical tablets via formation of a ternary complex with eosin and palladium (II) which showed an absorption maximum at $545 \mathrm{~nm}$ [191]. Ofloxacin generates with $\mathrm{Al}(\mathrm{III})$ and erythrosin an ion-association complex between $\left\{\mathrm{Al}^{\mathrm{III}}(\mathrm{OFX})\right\}$ cation and (ERY) anion. The ternary complex has an effective molar absorptivity at $555 \mathrm{~nm}$, allowed spectrophotometrically determination of ofloxacin and other quinolone antibiotics (norfloxacin, enoxacin and levofloxacin) in pharmaceutical preparation [192]. Ion-association complexes formed with $\left[\mathrm{Cr}(\mathrm{NCS})_{4}\left(\mathrm{NH}_{3}\right)_{2}\right]^{-}$(Reineckate anion) displaying a maximum absorption at $524 \mathrm{~nm}$ were used for determination of ofloxacin [193] and norfloxacin [194].

A spectrophotometric method related to the interaction of quinolones with metal ions was developed based on the oxidation of quinolones with ammonium vanadate in sulphuric acid medium, followed by the development of a greenish blue colour measured at $766 \mathrm{~nm}$, which has been attributed 
to vanadium(IV). The method was applied for determination of amifloxacin, ciprofloxacin, difloxacin, enoxacin, enrofloxacin, lomefloxacin, levofloxacin, norfloxacin, ofloxacin and pefloxacin in pharmaceutical dosage forms [195].

Modification of the fluorescent properties of quinolones in the presence of different metal ions has attracted the interest for studying the interaction of quinolones with antacids [196] and for development of spectrofluorimetric methods, applied in determination of quinolones in bulk, in biological fluids and in pharmaceutical formulations.

Determination of quinolones by spectrofluorimetric methods is based on: (i) the enhancement of quinolone fluorescence in the presence of metal ions (i.e., $\mathrm{Al}^{3+}, \mathrm{Cu}^{2+}, \mathrm{Au}^{3+}$ etc.); (ii) fluorescence sensitization of $\mathrm{Tb}^{3+}$ or $\mathrm{Eu}^{3+}$ in the presence of quinolone or (iii) quenching the fluorescence of a $\mathrm{Tb}^{3+}$ chelate after the addition of quinolone.

Interaction of a series of quinolones (sparfloxacin, oxolinic acid, flumequine and enrofloxacin) with $\mathrm{Al}^{3+}$ was used to analyse them in pharmaceutical dosage forms or in biological fluids [197]. Norfloxacin has been also determined as its fluorescent complex with $\mathrm{Al}^{3+}$ in serum [198] and in pharmaceutical preparations [199].

Formation of Y(III) fluorescent complexes underlying spectrofluorimetric methods for determination of norfloxacin in eye drops [200] and enrofloxacin in pharmaceutical formulations and its residue in milk [201].

The enhancement of luminescent properties of $\mathrm{Tb}$ (III) sorbates with ciprofloxacin and norfloxacin in zeolite was used for determination of these quinolones in biological fluids [202]. The enhancement effect of some quinolones on the fluorescence intensity of $\mathrm{Tb}$ (III)-sodium dodecylbenzenesulfonate system allowed the determination of enoxacin in pharmaceutical samples [203] and danofloxacin in milk [204]. Based on the sensitized fluorescence of $\mathrm{Tb}(\mathrm{III})$ enhanced by silver nanoparticles ciprofloxacin was dosed in pharmaceutical formulations [205], whereas pipemidic acid and lomefloxacin have been determined in pharmaceutical forms, urine and serum samples [206]. Europium (III)-sensitized fluorescence in the presence of quinolones was also applied for determination of quinolones ciprofloxacin, norfloxacin and gatifloxacin in pharmaceutical and serum samples [207] as for determination of ulifloxacin, the active metabolite of prulifloxacin in human serum and urine [208]. An optical sensor using $\mathrm{Tb}$ (III) and $\mathrm{Eu}(\mathrm{III})$ was constructed for analysis of norfloxacin and gatifloxacin in pharmaceutical and serum samples [209].

Quencing the fluorescence of an $\mathrm{Eu}(\mathrm{III})-\beta$-diketone complex in micellar solution after the addition of pefloxacin underlying a time-resolved fluorimetric method for determination of pefloxacin in serum [210].

Apart from the main analytical applications in determination of quinolones in pharmaceutical forms and in biological samples, the fluorescent complexes were used also for other purposes. In this regard, fluorescence studies of $\mathrm{Au}(\mathrm{III})$-norfloxacin system were carried out in order to study the association of $\mathrm{Au}^{3+}$ ions with cationic, zwitterionic and anionic forms of the drug [211]. $\mathrm{Cu}$ (II)-ofloxacin interaction, studied by means of ofloxacin fluorescence quenching experiments in the presence of $\mathrm{Cu}(\mathrm{II})$, was evaluated for its environmental impact [212].

Forming the metal complexes was the basis of some indirect methods for analysis of quinolones using atomic absorption spectrometry (AAS). Flow injection-fast atomic absorption spectroscopy (FI-AAS) was applied for determination of norfloxacin based on the complexation reaction with $\mathrm{Fe}(\mathrm{III})$, via measuring the absorbance of $\mathrm{Fe}^{3+}$ [213]. The formation of ion associated in the presence of 
cobalt sulphate was used for AAS determination of some fluoroquinolones in pharmaceutical dosage forms and biological fluids [195]. Ion-pair complexes formed with Reineckate anion allowed AAS determination of gatifloxacin, moxifloxacin and sparfloxacin in pharmaceutical formulations [214].

\subsubsection{Determination of Metal Ions Based on Complexation with Quinolones}

Spectrophotometric and spectroflurimetric methods were developed for determination of metal ions based on their complexation with quinolones. Formation of a coloured chelate with norfloxacin, which exhibits an absorption maximum at $377 \mathrm{~nm}$, was used for development of a spectophotometric method for determination of trace amounts of Fe(III) [215].

Norfloxacin was used as reagent for determination of neodymium, holmium and erbium in mixed rare earth through a derivative spectrophotometric method, based on the enhancement of absorption at $575 \mathrm{~nm}$ for neodymium, $450 \mathrm{~nm}$ for holmium, and 523 for erbium, respectively [216].

The complex between europium(III) and gatifloxacin in a co-luminiscence system $\mathrm{Eu}^{3+}-\mathrm{La}^{3+}$ gatifloxacin-sodium dodecylbenzene sulfonate was used for the determination of trace amounts of $\mathrm{Eu}^{3+}$ in rare earth samples [217]. Quencing fluorescence of a terbium chelates in the presence of $\mathrm{Hg}^{2+}$ was used for development of a highly sensitive and specific detection method of trace $\mathrm{Hg}^{2+}$ in trace $\mathrm{Hg}^{2+}$ in biological samples (urine) and environmental water [218].

\subsubsection{Quinolone Metal Complexes as Labels or Probes for Various Purposes}

The luminescent properties of $\mathrm{Tb}(\mathrm{III})$ and $\mathrm{Eu}(\mathrm{III})$ chelates of some quinolones (nalidixic acid, oxolinic acid, pipemidic acid, pefloxacin, norfloxacin, ofloxacin, ciprofloxacin and lomefloxacin) were characterized and the obtained reagents were proposed as labels for immunofluorimetric assay [219]. Based on the enhancement of the fluorescence intensity of the enoxacin- $\mathrm{Tb}^{3+}$ complex, an environmentally friendly probe for determination of DNA (both single-stranded and double-stranded) was developed [220].

\section{Conclusions}

The metal ion - quinolone complexation represents a research field of increasing progress, having in view the consequences and applications of this process. Pharmaceutical profiles of quinolones can be improved by obtaining complexes with enhanced solubility. On the other side, pharmacokinetic interactions can occur at oral co-administration of quinolones and metal ions from mineral supplements and antacids. At the target site of their action, a quinolone-gyrase-DNA complex is formed in the presence of $\mathrm{Mg}^{2+}$ ions.

Many metal ion - quinolone complexes obtained in the solid state have shown various biological effects: antimicrobial activity (sometimes equal or better than that of the parent quinolone), anticancer activity, and, in some cases, antifungal and antiparasitic activity.

Complexation with metal ions was harnessed in the development of spectrophotometric, spectroflurimetric and atomic absorbtion spectrometric methods for the determination of quinolones in pharmaceutical preparations or in biological samples. Conversely, trace metal ions can be determined using quinolones as complexing agents. It must be noted that the progresses in the field of quinolone 
complexes and their applications parallel the development of the newer fluoroquinolones with enlarged biological activity.

\section{Acknowledgments}

This work was supported by a grant of the Romanian National Authority for Scientific Research, CNDI-UEFISCDI, project number 136/2012. I would like to express my special gratitude to the National Electronic Access to Scientific Research Literature (ANELiS) Project financed by the European Regional Development Fund who gave me the opportunity to do this work, which also helped me in doing a lot of research and I came to know about so many new things. I am really thankful to them.

\section{Conflicts of Interest}

The author declares no conflict of interest.

\section{References}

1. Appelbaum, P.C.; Hunter, P.A. The fluoroquinolone antibacterials: Past, present and future perspectives. Int. J. Antimicrob. Agents 2000, 16, 5-15.

2. Hooper, D.C. Clinical application of quinolones. Biochim. Biophys. Acta-Gene Struct. Express. 1998, 1400, 45-61.

3. Buchbinder, M.; Webb, J.C.; Anderson, L.V.; McCabe, W.R. Laboratory studies and clinical pharmacology of nalidixic acid (WIN 18, 320). Antimicrob. Agents Chemother. 1962, 2 , 308-317.

4. Brighty, K.E.; Gootz, T.D. Chemistry and Mechanism of Action of the Quinolone Antibacterials. In The Quinolones, 3rd ed.; Andriole, V.T., Ed.; Academic Press: San Diego, CA, USA, 2000; pp. 33-97.

5. Senf, H.J. Fluorochinolone (Gyrasehemmer). Pharmazie 1988, 43, 444-447.

6. Smith, J.T.; Lewin, C.S. Chemistry and Mechanisms of Action of the Quinolone Antibacterials. In The Quinolones; Andriole, V.T., Ed.; Academic Press: London, UK, 1988; pp. 23-81.

7. Oliphant, C.M.; Green, G.M. Quinolones: A comprehensive review. Am. Fam. Phys. 2002, 65, 455-464.

8. King, D.E.; Malone, R.; Lilley, S.H. New classification and update on the quinolone antibiotics. Am. Fam. Phys. 2000, 61, 2741-1748.

9. Zhanel, G.G.; Walkty, A.; Vercaigne, L.; Karlowsky, J.A.; Embil, J.; Gin, A.S.; Hoban, D.J. The new fluoroquinolones: A critical review. Can. J. Infect. Dis. 1999, 10, 207-238.

10. Cozzarelli, N.R. DNA gyrase and the supercoiling of DNA. Science 1980, 207, 953-960.

11. Mitscher, L.A. Bacterial topoisomerase inhibitors: Quinolone and pyridone antibacterial agents. Chem. Rev. 2005, 105, 559-592.

12. Blondeau, J.M. Fluoroquinolones: Mechanism of action, classification, and development of resistance. Surv. Ophthalmol. 2004, 49, S73-S78. 
13. Drlica, K.; Zhao, X. DNA gyrase, topoisomerase IV, and the 4-quinolones. Microbiol. Mol. Biol. Rev. 1997, 61, 377-392.

14. Hooper, D.C. Mechanisms of action and resistance of older and newer fluoroquinolones. Clin. Infect. Dis. 2000, 31, S24-S28.

15. Maxwell, A. The molecular basis of quinolone action. J. Antimicrob. Chemother. 1992, 30, 409-414.

16. Schaumann, R.; Rodloff, A.C. Activities of quinolones against obligately anaerobic bacteria. Anti-Infective Agents Med. Chem. 2007, 6, 49-56.

17. Shen, L.L.; Chu, D.T.W. Type II DNA topoisomerases as antibacterial targets. Curr. Pharm. Des. 1996, 2, 195-208.

18. Peterson, L.R. Quinolone molecular structure-activity relationships: What we have learned about improving antimicrobial activity. Clin. Infect. Dis. 2001, 33, S180-S186.

19. Ross, D.; Riley, C. Physicochemical properties of the fluoroquinolone antimicrobials. II. Acid ionization constants and their relationship to structure. Int. J. Pharmaceut. 1992, 83, 267-272.

20. Takacs-Novak, K.; Noszal, B.; Hermecz, I.; Kereszturi, G.; Podanyi, B.; Szasz, G. Protonation equilibria of quinolone antibacterials. J. Pharm. Sci. 1990, 79, 1023-1028.

21. Turel, I. The interactions of metal ions with quinolone antibacterial agents. Coord. Chem. Rev. 2002, 232, 27-47.

22. Zupančič, M.; Cerc Korošec, R.; Bukovec, P. The thermal-stability of ciprofloxacin complexes with magnesium (II), zinc (II) and cobalt (II). J. Therm. Anal. Calorim. 2001, 63, 787-795.

23. Sasz, G.; Takacs-Novak, K.; Budvari-Barany, S.; Hermecz, J.; Jozan, M.; Lore, A.; Noszal, B. Correlation between the structures and physicochemical properties of chemoterapeutic fluoroquinolone agents. Acta Pharm. Hung. 1993, 63, 105-114.

24. Turel, I.; Bukovec, N.; Farkas, E. Complex formation between some metals and a quinolone family member (ciprofloxacin). Polyhedron 1996, 15, 269-275.

25. Ma, H.; Chiu, F.; Li, R. Mechanistic investigation of the reduction in antimicrobial activity of ciprofloxacin by metal cations. Pharm. Res. 1997, 14, 366-370.

26. El-Roudi, A.M.; Soliman, E.M.; Refaiy, S.A. Effect of substituent and solvent composition on the stability of the metal complexes of 2-quinolone derivatives. Afinidad 1989, 420, 154-156.

27. Ross, D.; Riley, C. Physicochemical properties of the fluoroquinolone antimicrobials. V. Effect of fluoroquinolones structure and $\mathrm{pH}$ on the complexation of various fluoroquinolones with magnesium and calcium ions. Int. J. Pharmaceut. 1993, 93, 121-129.

28. Efthimiadou, E.K.; Sanakis, Y.; Katsaros, N.; Karaliota, A.; Psomas G. Transition metal complexes with the quinolone antibacterial agent pipemidic acid: Synthesis, characterization and biological activity. Polyhedron 2007, 26, 1148-1158.

29. Efthimiadou, E.K.; Katsaros, N.; Karaliota A.; Psomas G. Mononuclear copper(II) complexes with quinolones and nitrogen-donor heterocyclic ligands: Synthesis, characterization, biological activity and interaction with DNA. Inorg. Chim. Acta 2007, 360, 4093-4102.

30. Skrzypek, D.; Szymanska, B.; Kovala-Demertzi, D.; Wiecek, J.; Talik, E.; Demertzis, M.A. Synthesis and spectroscopic studies of iron (III) complex with a quinolone family member (pipemidic acid). J. Phys. Chem. Solids 2006, 67, 2550-2558. 
31. Ruíz, M.; Ortiz, R.; Perelló, L.; Latorre, J.; Server-Carrio, J. Potentiometric and spectroscopic studies of transition-metal ions complexes with a quinolone derivative (cinoxacin). Crystal structures of new $\mathrm{Cu}$ (II) and Ni (II) cinoxacin complexes. J. Inorg. Biochem. 1997, 65, 87-96.

32. Ruíz, M.; Perelló, L.; Ortiz, R.; Castineiras, A.; Maichle-Mossmer, C.; Canton, E. Synthesis, characterization and crystal structure of $\left[\mathrm{Cu}(\mathrm{Cinoxacinate})_{2}\right] 2 \mathrm{H}_{2} \mathrm{O}$ complex: A square planar $\mathrm{CuO}_{4}$ cromophore. Antibacterial studies. J. Inorg. Biohem. 1995, 59, 801-810.

33. Ruíz, M.; Perelló, L.; Server-Carrio, J.; Ortiz, R.; Garcia-Granda, S.; Diaz, M.R.; Canton, E. Cinoxacin complexes with divalent metal ions. Spectroscopic characterization. Crystal structure of a new dinuclear Cd (II) complex having two chelate-bridging carboxylate groups. Antibacterial studies. J. Inorg. Biochem. 1998, 69, 231-239.

34. Lopez-Gresa, M.P.; Ortiz, R.; Perelló, L.; Latorre, J.; Liu-González, M.; García-Granda, S.; Pérez-Priede, M.; Canton, E. Interaction of metal ions with two quinolone antimicrobial agents (cinoxacin and ciprofloxacin). Spectroscopic and X-ray structural characterization. Antibacterial studies. J. Inorg. Biochem. 2002, 92, 65-74.

35. Psomas, G.; Tarushi, A.; Efthimiadou, E.K.; Sanakis, Y.; Raptopoulou, C.P.; Katsaros, N. Synthesis, structure and biological activity of copper(II) complexes with oxolinic acid. J. Inorg. Biochem. 2006, 100, 1764-1773.

36. Skyrianou K.C.; Perdih, F.; Turel, I.; Kessissoglou, D.P.; Psomas, G. Nickel-quinolones interaction. Part 2 - Interaction of nickel(II) with the antibacterial drug oxolinic acid. J. Inorg. Biochem. 2010, 104, 161-170.

37. Tarushi, A.; Psomas, G.; Raptopoulou, C.P.; Kessissoglou, D.P. Zinc complexes of the antibacterial drug oxolinic acid: Structure and DNA-binding properties. J. Inorg. Biochem. 2009, 103, 898-905.

38. Tarushi, A.; Christofis, P.; Psomas, G. Synthesis, characterization and interaction with DNA of mononuclear metal complexes with oxolinic acid. Polyhedron 2007, 26, 3963-3972.

39. Tarushi, A.; Efthimiadou, E.K.; Christofis, P.; Psomas, G. Neutral mononuclear dioxomolybdenum(VI) and dioxouranium(VI) complexes of oxolinic acid: Characterization and biological evaluation. Inorg. Chim. Acta 2007, 360, 3978-3986.

40. Perez-Guaita, D.; Boudesocque, S.; Sayen, S.; Guillon, E. Cu(II) and Zn(II) complexes with a fluoroquinolone antibiotic: Spectroscopic and X-ray absorption characterization. Polyhedron 2011, 30, 438-443.

41. Chalkidou, E.; Perdih, F.; Turel, I.; Kessissoglou, D.P.; Psomas, G. Copper(II) complexes with antimicrobial drug flumequine: Structure and biological evaluation. J. Inorg. Biochem. 2012, 113, 55-65.

42. Skyrianou, K.C.; Perdih, F.; Turel, I.; Kessissoglou, D.P.; Psomas, G. Nickel-quinolones interaction Part 3 - Nickel(II) complexes of the antibacterial drug flumequine. J. Inorg. Biochem. 2010, 104, 740-749.

43. Tarushi, A.; Kljun, J.; Turel, I.; Pantazaki, A.A.; Psomas, G.; Kessissoglou, D.P. Zinc(II) complexes with the quinolone antibacterial drug flumequine: Structure, DNA- and albumin-binding. New J. Chem. 2013, 37, 342-355. 
44. Jiménez-Garrido, N.; Perelló, L.; Ortiz R.; Alzuet, G.; González-Álvarez, M.; Cantón, E.; Liu-González, M.; García Granda, S.; Pérez-Priede, M. Antibacterial studies, DNA oxidative cleavage, and crystal structures of $\mathrm{Cu}$ (II) and $\mathrm{Co}$ (II) complexes with two quinolone family members, ciprofloxacin and enoxacin. J. Inorg. Biochem. 2005, 99, 677-689.

45. Arayne, S.; Sultana, N.; Haroon, U.; Mesaik, M.A. Synthesis, characterization, antibacterial and anti-inflammatory activities of enoxacin metal complexes. Bioinorg. Chem. Appl. 2009, doi:10.1155/2009/914105.

46. Sha, J.-Q.; Li, X.; Qiu, H.-B.; Zhang, Y.-H.; Yan, H. Nickel complexes of the different quinolone antibacterial drugs: Synthesis, structure and interaction with DNA. Inorg. Chim. Acta 2012, 383, 178-184.

47. Al-Mustafa, J. Magnesium, calcium and barium perchlorate complexes of ciprofloxacin and norfloxacin. Acta Chim. Slov. 2002, 49, 457-466.

48. Breda, S.A.; Jimenez-Kairuz, A.F.; Manzo, R.H.; Olivera, M.E. Solubility behavior and biopharmaceutical classification of novel high-solubility ciprofloxacin and norfloxacin pharmaceutical derivatives. Int. J. Pharmaceut. 2009, 371, 106-113.

49. Shaikh, A.R.; Giridhar, R.; Yadav M.R. Bismuth-norfloxacin complex: Synthesis, physicochemical and antimicrobial evaluation. Int. J. Pharmaceut. 2007, 332, 24-30.

50. Shaikh, A.R.; Giridhar, R.; Megraud, F.; Yadav, M.R. Metalloantibiotics: Synthesis, characterization and antimicrobial evaluation of bismuth-fluoroquinolone complexes against Helicobacter Pylori. Acta Pharm. 2009, 59, 259-271.

51. Sadeek, S.A. Synthesis, thermogravimetric analysis, infrared, electronic and mass spectra of $\mathrm{Mn}(\mathrm{II}), \mathrm{Co}(\mathrm{II})$ and Fe(III) norfloxacin complexes. J. Mol. Struct. 2005, 753, 1-12.

52. Golovnev, N.N.; Kirik, S.D.; Golovneva, I.I. Synthesis of norfloxacin compounds with cobalt(II), zinc(II), cadmium(II), and mercury(II). Russ. J. Inorg. Chem. 2009, 54, 223-225.

53. Batista, D.G.J.; da Silva, P.B.; Stivanin, L.; Lachter, D.R.; Silva, R.S.; Felcman, J.; Louro, S.R.W.; Teixeira, L.R.; de Nazare C. Soeiro, M. Co(II), Mn(II) and Cu(II) complexes of fluoroquinolones: Synthesis, spectroscopical studies and biological evaluation against Trypanosoma cruzi. Polyhedron 2011, 30, 1718-1725.

54. Ruíz, P.; Ortiz, R.; Perelló, L.; Alzuet, G.; González-Álvarez, M.; Liu-González, M.; Sanz-Ruíz, F. Synthesis, structure, and nuclease properties of several binary and ternary complexes of copper(II) with norfloxacin and 1,10 phenantroline. J. Inorg. Biochem. 2007, 101, 831-840.

55. Živec, P.; Perdih, F.; Turel, I.; Giester, G.; Psomas, G. Different types of copper complexes with the quinolone antimicrobial drugs ofloxacin and norfloxacin: Structure, DNA- and albumin-binding. J. Inorg. Biochem. 2012, 117, 35-47.

56. Zhang, J.J.; Ge, L.G.; Zhang, X.L.; Dai, Y.J.; Chen, H.L.; Mo, L.P. Thermal decomposition kinetics of the $\mathrm{Zn}(\mathrm{II})$ complex with norfloxacin in static air atmosphere. J. Therm Anal. Calorim. 1999, 58, 269-278.

57. Refat, M.S.; Mohamed, G.G.; de Farias, R.F.; Powell, A.K.; El-Garib, M.S.; El-Korashy, S.A.; Hussien, M.A. Spectroscopic, thermal and kinetic studies of coordination compounds of $\mathrm{Zn}$ (II), $\mathrm{Cd}(\mathrm{II})$ and $\mathrm{Hg}(\mathrm{II})$ with norfloxacin. J. Therm. Anal. Calorim. 2010, 102, 225-232. 
58. Sadeek, S.A.; El-Did Amony, A.M.; El-Shwiniy, W.H.; Zordok, W.A. Uranium (VI) and zirconium (IV) of the second -generation quinolone antimicrobial drug norfloxacin: Structure and biological activity. J. Argent. Chem. Soc. 2009, 97, 51-76.

59. Chen, X.-B.; Ye, Q.; Wu, Q.; Song, Y.-M.; Xiong R.-G.; You, X.-Z. The first organometallic carbonyl tungsten complex of antibacterial drug norfloxacin. Inorg. Chem. Commun. 2004, 7, 1302-1305.

60. Uivarosi, V.; Badea, M.; Olar, R.; Marinescu, D.; Nicolescu, T.O.; Nitulescu, G.M. Thermal degradation behavior of some ruthenium complexes with fluoroquinolone derivatives as potential antitumor agents. J. Therm. Anal. Calorim. 2011, 105, 645-650.

61. Patel, M.N.; Gandhi, D.S.; Parmar, P.A. DNA interaction and in-vitro antibacterial studies of fluoroquinolone based platinum(II) complexes. Inorg. Chem. Commun. 2012, 15, 248-251.

62. Gouvea, L.R.; Garcia, L.S.; Lachter, D.R.; Nunes, P.R.; de Castro Pereira, F.; Silveira-Lacerda, E.P.; Louro, S.R.W. Barbeira, P.J.S.; Teixeira, L.R. Atypical fluoroquinolone gold(III) chelates as potential anticancer agents: Relevance of DNA and protein interactions for their mechanism of action. Eur. J. Med. Chem. 2012, 55, 67-73.

63. Sadeek, S.A.; El-Shwiniy, W.H.; Zordok, W.A.; El-Didamony, A.M. Synthesis, spectroscopic, thermal and biological activity investigation of new Y(III) and $\mathrm{Pd}(\mathrm{II})$ norfloxacin complexes. J. Argent. Chem. Soc. 2009, 97, 128-148.

64. Refat, M.S.; El-Hawary, W.F.; Mohamed, M.A. Study of the chemical chelates and anti-microbial effect of some metal ions in nanostructural form on the efficiency of antibiotic therapy "norfloxacin drug". J. Mol. Struct. 2012, 1013, 45-54.

65. Li, S.; Wang Y.; Lin, Q.; Liu, W.; Ding, J.; Wang, Y. Synthesis, crystal structures of novel complexes of rare earth with norfloxacin, interaction with DNA and BSA. J. Rare Earths 2012 , 30, 460-466.

66. Qi, W.; Huang, J.; An, Z. Aquabis[1-ethyl-6-fluoro-7-(4-methylpiperazin-1-yl)-4-oxo-1,4dihydroquinoline-3-carboxylato]zinc(II) dihydrate. Acta Crystallogr. 2008, 64, m302.

67. Drevenšek, P.; Poklar Ulrih, N.; Majerle, A.; Turel, I. Synthesis, characterization and DNA binding of magnesium-ciprofloxacin ( $\mathrm{cfH})$ complex $\left[\mathrm{Mg}(\mathrm{cf})_{2}\right] \cdot 2.5 \mathrm{H}_{2} \mathrm{O}$. J. Inorg. Biochem. 2006, 100, 1705-1713.

68. Turel, I.; Šonc, A.; Zupančič, M.; Sepčić, K.; Turk, T. Biological activity of some magnesium(II) complexes of quinolones. Met. Based Drugs 2000, 7, 101-104.

69. Turel, I.; Živec, P.; Pevec, A.; Tempelaar, S.; Psomas, G. Compounds of antibacterial agent ciprofloxacin and magnesium - crystal structures and molecular modeling calculations. Eur. J. Inorg. Chem. 2008, 23, 3718-3727.

70. Al-Mustafa, J.; Taha, Z.A. Thermodynamics of the complexation of ciprofloxacin with calcium and magnesium perchlorate. Thermochim. Acta 2011, 521, 9-13.

71. Turel I.; Golobič, A.; Klavžar, A.; Pihlar, B.; Buglyó, P.; Tolis, E.; Rehder, D.; Sepčić, K. Interactions of oxovanadium(IV) and the quinolone family member-ciprofloxacin. J. Inorg. Biochem. 2003, 95, 199-207.

72. Anacona, J.R.; Toledo, C. Synthesis and antibacterial activity of metal complexes of ciprofloxacin. Trans. Met. Chem. 2001, 26, 228-231. 
73. Psomas, G. Mononuclear metal complexes with ciprofloxacin: Synthesis, characterization and DNA-binding properties. J. Inorg. Biochem. 2008, 102, 1798-1811.

74. Hernandez-Gil, J.; Perello, L.; Ortiz, R.; Alzuet, G.; Gonzalez-Alvarez, M.; Liu-Gonzalez, M. Synthesis, structure and biological properties of several binary and ternary complexes of copper(II) with ciprofloxacin and 1,10 phenanthroline. Polyhedron 2009, 28, 138-144.

75. Wallis, S.C.; Gahan, L.R.; Charles, B.G.; Hambley, T.W.; Duckworth, P.A. Copper (II) complexes of the fluoroquinolone antimicrobial ciprofloxacin: Synthesis, X-ray structural characterization and potentiometric study. J. Inorg. Biochem. 1996, 62, 1-16.

76. Turel, I.; Leban, I.; Bukovec, N. Synthesis, characterization, and crystal structure of a copper(II) complex with quinolone family member (ciprofloxacin): Bis(1)-cyclopropyl-6-fluoro1,4-dihydro-4-oxo-7-piperazin-1ylquinoline-3-carboxylate) copper(II) chloride hexahydrate. J. Inorg. Biochem. 1994, 56, 273-282.

77. Drevenšek, P.; Zupančič, T.; Pihlar, B.; Jerala, R.; Kolitsch, U.; Plaper, A.; Turel, I. Mixed-valence $\mathrm{Cu}(\mathrm{II}) / \mathrm{Cu}(\mathrm{I})$ complex of quinolone ciprofloxacin isolated by a hydrothermal reaction in the presence of L-histidine: Comparison of biological activities of various copper-ciprofloxacin compounds. J. Inorg. Biochem. 2005, 99, 432-442.

78. Tanimoto, M.K; Dias, K.; Dovidauskas, S.; Nikolaou, S. Tuning the reaction products of ruthenium and ciprofloxacin for studies of DNA interactions. J. Coord Chem. 2012, 65, 1504-1517.

79. Vieira, L.M.M.; de Almeida, M.V.; Lourenço, M.C.S.; Bezerra, F.A.F.M.; Fontes, A.P.S. Synthesis and antitubercular activity of palladium and platinum complexes with fluoroquinolones. Eur. J. Med. Chem. 2009, 44, 4107-4111.

80. Čurman, D.; Živec, P.; Leban, I.; Turel, I.; Polishchuk, A.; Klika, K.D.; Karaseva, E.; Karasev, V. Spectral properties of $\mathrm{Eu}(\mathrm{III})$ compound with antibacterial agent ciprofloxacin (cfqH). Crystal structure of $\left[\mathrm{Eu}(\mathrm{cfqH})(\mathrm{cfq})\left(\mathrm{H}_{2} \mathrm{O}\right)_{4}\right] \mathrm{Cl}_{2} \cdot 4.55 \mathrm{H}_{2} \mathrm{O}$. Polyhedron 2008, 27, 1489-1496.

81. Sadeek, S.A.; El-Shwiniy, W.H. Preparation, structure and microbial evaluation of metal complexes of the second generation quinolone antibacterial drug lomefloxacin. J. Mol. Struct. 2010, 98, 130-138.

82. Abd El-Halim, H.F.; Mohamed, G.G.; El-Dessouky, M.M.I.; Mahmoud, W.H. Ligational behaviour of lomefloxacin drug towards $\mathrm{Cr}(\mathrm{III}), \mathrm{Mn}(\mathrm{II}), \mathrm{Fe}(\mathrm{III}), \mathrm{Co}(\mathrm{II}), \mathrm{Ni}(\mathrm{II}), \mathrm{Cu}(\mathrm{II}), \mathrm{Zn}(\mathrm{II})$, $\mathrm{Th}(\mathrm{IV})$ and $\mathrm{UO}_{2}$ (VI) ions: Synthesis, structural characterization and biological activity studies. Spectrochim. Acta A 2011, 82, 8-19.

83. Drevenšek, P.; Košmrlj, J.; Giester, G.; Skauge, T.; Sletten, E.; Sepčić, K.; Turel, I. X-ray crystallographic, NMR and antimicrobial activity studies of magnesium complexes of fluoroquinolones - racemic ofloxacin and its S-form, levofloxacin. J. Inorg. Biochem. 2006, 100, $1755-1763$.

84. Sagdinc, S.; Bayari, S. Spectroscopic studies on the interaction of ofloxacin with metals. J. Mol. Struct. 2004, 691, 107-113.

85. Macias, B.; Villa, M.V.; Sastre, M.; Castiñeiras, A.; Borras, J. Complexes of Co(II) and Zn(II) with ofloxacin. Crystal structure of $\left[\mathrm{Co}(\text { oflo })_{2}(\mathrm{MeOH})_{2}\right] \cdot 4 \mathrm{MeOH}$. J. Pharm. Sci. 2002, 91, 2416-2423. 
86. Macias, B.; Villa, M.; Rubio, I.; Castineiras, A.; Borras, J. Complexes of Ni (II) and Cu (II) with ofloxacin. Crystal structure of a new $\mathrm{Cu}$ (II) ofloxacin complex. J. Inorg. Biochem. 2001, 84, $163-170$.

87. Xu, M.; Zhang, Y.-C.; Xu, Z.-H.; Zeng, Z.-Z. Crystal structure, biological studies of water-soluble rare earth metal complexes with an ofloxacin derivative. Inorg. Chim. Acta 2012, 384, 324-332.

88. Efthimiadou, E.K.; Katsaros, N.; Karaliota, A.; Psomas, G. Synthesis, characterization, antibacterial activity, and interaction with DNA of the vanadyl-enrofloxacin complex. Bioorg. Med. Chem. Lett. 2007, 17, 1238-1242.

89. Efthimiadou, E.K.; Karaliota, A.; Psomas, G. Mononuclear dioxomolybdenum(VI) complexes with the quinolones enrofloxacin and sparfloxacin: Synthesis, structure, antibacterial activity and interaction with DNA. Polyhedron 2008, 27, 349-356.

90. Efthimiadou, E.K.; Karaliota, A.; Psomas, G. Mononuclear metal complexes of the second-generation quinolone antibacterial agent enrofloxacin: Synthesis, structure, antibacterial activity and interaction with DNA. Polyhedron 2008, 27, 1729-1738.

91. Skyrianou, K.C.; Psycharis, V.; Raptopoulou, C.P.; Kessissoglou, D.P.; Psomas, G. Nickel-quinolones interaction. Part 4 - Structure and biological evaluation of nickel(II)enrofloxacin complexes compared to zinc(II) analogues. J. Inorg. Biochem. 2011, 105, 63-74.

92. Saraiva, R.; Lopes, S.; Ferreira, M.; Novais, F.; Pereira, E.; Feio, M.J.; Gameiro, P. Solution and biological behaviour of enrofloxacin metalloantibiotics: A route to counteract bacterial resistance? J. Inorg. Biochem. 2010, 104, 843-850.

93. Efthimiadou, E.K.; Sanakis, Y.; Katsarou, M.; Raptopoulou, C.P.; Karaliota, A.; Katsaros, N.; Psomas, G. Neutral and cationic mononuclear copper(II) complexes with enrofloxacin: Structure and biological activity. J. Inorg. Biochem. 2006, 100, 1378-1388.

94. Ftouni, H.; Sayen, S.; Boudesocque, S.; Dechamps-Olivier, I.; Guillon, E. Structural study of the copper(II)-enrofloxacin metallo-antibiotic. Inorg. Chim. Acta 2012, 382, 186-190.

95. Efthimiadou, E.K.; Karaliota, A.; Psomas, G. Metal complexes of the third-generation quinolone antimicrobial drug sparfloxacin: Structure and biological evaluation. J. Inorg. Biochem. 2010, 104, 455-466.

96. Efthimiadou, E.K.; Karaliota, A.; Psomas, G. Structure, antimicrobial activity and DNA-binding properties of the cobalt(II)-sparfloxacin complex. Bioorg. Med. Chem. Lett. 2008, 18, 4033-4037.

97. Efthimiadou, E.K.; Sanakis, Y.; Raptopoulou, C.P.; Karaliota, A.; Katsaros, N.; Psomas, G. Crystal structure, spectroscopic, and biological study of the copper(II) complex with third-generation quinolone antibiotic sparfloxacin. Bioorg. Med. Chem. Lett. 2006, 16, 3864-3867.

98. Sultana, N.; Arayne, M.S.; Rizvi, S.B.S.; Haroon, U.; Mesaik, M.A. Synthesis, spectroscopic, and biological evaluation of some levofloxacin metal complexes. Med. Chem. Res. 2013, 22, 1371-1377.

99. Tarushi, A.; Polatoglou, E.; Kljun, J.; Turel, I.; Psomas, G.; Kessissoglou, D.P. Interaction of $\mathrm{Zn}(\mathrm{II})$ with quinolone drugs: Structure and biological evaluation. Dalton Trans. 2011, 40, 9461-9473. 
100. Sultana, N.; Naz, A.; Arayne, M.S.; Ahmed Mesaik, M. Synthesis, characterization, antibacterial, antifungal and immunomodulating activities of gatifloxacin-metal complexes. J. Mol. Struct. 2010, 969, 17-24.

101. Li, Z.-Q.; Wu, F.-J.; Gong, Y.; Hu, C.-W.; Zhang, Y.-H.; Gan, M.-Y. Synthesis, characterization and activity against Staphylococcus of metal(II)-gatifloxacin complexes. Chin. J. Chem. 2007, 25, 1809-1814.

102. Mehrotra, R.; Shukla, S.N., Gaur, P.; Dubey, A. Identification of pharmacophore in bioactive metal complexes: Synthesis, spectroscopic characterization and application. Eur. J. Med. Chem. 2012, 50, 149-153.

103. Patitungkho, S.; Adsule, S.; Dandawate, P.; Padhye, S.; Ahmad, A.; Sarkar, F.H. Synthesis, characterization and anti-tumor activity of moxifloxacin-copper complexes against breast cancer cell lines. Bioorg. Med. Chem. Lett. 2011, 21, 1802-1806.

104. Sadeek, S.A.; El-Shwiniy, W.H.; El-Attar, M.S. Synthesis, characterization and antimicrobial investigation of some moxifloxacin metal complexes. Spectrochim. Acta Part A 2011, 84, 99-110.

105. Sadeek, S.A.; El-Shwiniy, W.H.; Zordok, W.A.; Kotb, E. Spectroscopic studies, thermal analyses and biological evaluation of new V(IV), Zr(IV) and U(VI) moxifloxacin complexes. J. Mol. Struct. 2011, 1006, 192-209.

106. Serafin, A.; Stanczak, A. The complexes of metal ions with fluoroquinolones. Russ. J. Coord. Chem. 2009, 35, 81-95.

107. Psomas, G.; Kessissoglou, D.P. Quinolones and non-steroidal antiinflammatory drugs interacting with copper(II), nickel(II), cobalt(II) and zinc(II): Structural features, biological evaluation and perspectives. Dalton Trans. 2013, 42, 6252-6276.

108. Gao, F.; Yang, P.; Xie, J.; Wang, H. Synthesis, characterization and antibacterial activity of novel Fe(III), Co(II), and Zn(II) complexes with norfloxacin. J. Inorg. Biochem. 1995, 60, 61-67.

109. Vieira, L.M.M.; de Almeida, M.V.; de Abreu, H.A.; Duarte, H.A.; Grazul, R.M.; Fontes, A.P.S. Platinum(II) complexes with fluoroquinolones: Synthesis and characterization of unusual metal-piperazine chelates. Inorg. Chim. Acta 2009, 362, 2060-2064.

110. Rusu, A.; Tóth, G.; Szőcs, L.; Kökösi, J.; Kraszni, M.; Gyéresi, A.; Noszál, B. Triprotic site-specific acid-base equilibria and related properties of fluoroquinolone antibacterials. J. Pharm. Biomed. Anal. 2012, 66, 50-57.

111. Sha, J.-Q.; Liang, L.-Y.; Yan, P.-F.; Li, G.-M.; Wang, C.; Ma, D.-Y. Study on ligation of copper complexes of the quinolone antibacterial drugs and octamolybdates POMs. Polyhedron 2012, 31, $422-430$.

112. Liu, Y.-C.; Chen, Z.-F.; Shi, S.-M.; Luo, H.-S.; Zhong, D.-C.; Zou, H.-L.; Liang, H. Synthesis, crystal structure of polyoxovanadate complex of ciprofloxacin: $\mathrm{V}_{4} \mathrm{O}_{10}\left(\mu_{2}-\mathrm{O}\right)_{2}\left[\mathrm{VO}(\mathrm{H}-\mathrm{Ciprof})_{2}\right]_{2}$. $13 \mathrm{H}_{2} \mathrm{O}$ by hydrothermal reaction. Inorg. Chem. Commun. 2007, 10, 1269-1272.

113. Li, C.; Lu, J.; Tu, F.; Chen, J.; Li, Y. Study of the first antibacterial agent pipemidic acid modifying Keggin polyoxometalate. Inorg. Chem. Commun. 2011, 14, 1192-1195. 
114. Sha, J.-Q.; Liang, L.-Y.; Li, X.; Zhang, Yu.; Yan, H.; Chen, G. Ligation of the quinolone antibacterial agent pipemidic acid to Keggin polyoxotungstates. Polyhedron 2011, 30, 1657-1662.

115. Sha, J.-Q.; Li, X.; Zhou, Y.-H., Yan, P.-F.; Li, G.-M.; Wang, C. The introduction of antibacterial drug pipemidic acid into the POM field: Syntheses, characterization and antitumor activity. Solid State Sci. 2011, 13, 1972-1977.

116. Li, Y.-X.; Chen, Z.-F.; Xiong, R.-G.; Xue, Z.; Ju, H.-X.; You, X.-Z. A mononuclear complex of norfloxacin with silver(I) and its properties. Inorg. Chem. Commun. 2003, 6, 819-822.

117. Refat, M.S. Synthesis and characterization of norfloxacin-transition metal complexes (group 11, IB): Spectroscopic, thermal, kinetic and biological activity. Spectrochim. Acta Part A 2007, 5, 1393-1405.

118. Badea, M.; Olar, R.; Marinescu, D.; Uivarosi, V.; Iacob, D. thermal decomposition of some biologically active complexes of ruthenium (III) with quinolone derivatives. J. Therm. Anal. Calorim. 2009, 97, 735-739.

119. Badea, M.; Olar, R.; Marinescu, D.; Uivarosi, V.; Nicolescu, T.O.; Iacob, D. Thermal study of some new quinolone ruthenium(III) complexes with potential cytostatic activity. J. Therm. Anal. Calorim. 2010, 99, 829-834.

120. Chen, Z.F.; Xiong, R.G.; Zuo, J.; Guo, Z.; You, X.; Fun, H.K. X-ray crystal structures of $\mathrm{Mg}^{2+}$ and $\mathrm{Ca}^{2+}$ dimers of the antibacterial drug norfloxacin. J. Chem. Soc. Dalton Trans. 2000, 22, 4013-4014.

121. Chen, Z.F.; Zhou, H.L.; Liang, H.; Li, Y.; Xiong, R.G.; You, X.Z. Crystallographic report: Bis(norfloxacin)dilead(II) tetranitrate, $\left[\mathrm{Pb}_{2}(\mathrm{H}-\mathrm{Norf})_{2}\left(\mathrm{ONO}_{2}\right)_{4}\right]$. Appl. Organomet. Chem. 2003, $17,883-884$.

122. Qu, Z.-R.; Zhao, H.; Xing, L.-X.; Wang, X.-S.; Chen, Z.-F.; Yu, Z.; Xiong, R.-G.; You, X.-Z. Two polymeric complexes of norfloxacin with iron(II) and their magnetic properties. Eur. J. Inorg. Chem. 2003, 16, 2920-2923.

123. Chen, Z.-F.; Yu, L.-C.; Zhong, D.-C.; Liang, H.; Zhu, X.-H.; Zhu, Z.-Y. An unprecedented 1D ladder-like silver (I) coordination polymer with ciprofloxacin. Inorg. Chem. Commun. 2006, 9, 839-843.

124. Gerasimenko, A.V.; Polishchuk, A.V.; Volkova, L.M.; Karaseva, E.T.; Karasev, V.E. Synthesis and structure of nalidixium tetrachloroantimonate monohydrate, $\left(\mathrm{C}_{12} \mathrm{H}_{13} \mathrm{~N}_{2} \mathrm{O}_{3}\right) \mathrm{SbCl}_{4}$. $\mathrm{H}_{2} \mathrm{O}$. Russ. J. Coord. Chem. 2008, 34, 8-13.

125. Gerasimenko, A.V.; Polishchuk, A.V.; Karaseva, E.T.; Karasev, V.E. Crystal Structure and Spectroscopic Properties of Ciprofloxacinium Pentachloroantimonate(III) Monohydrate $\left(\mathrm{C}_{17} \mathrm{H}_{19} \mathrm{~N}_{3} \mathrm{O}_{3} \mathrm{~F}\right) \mathrm{SbCl}_{5} \cdot \mathrm{H}_{2} \mathrm{O}$. Russ. J. Coord. Chem. 2008, 34, 647-652.

126. Turel, I.; Leban, I.; Bukovec, N. Crystal structure and characterization of the bismuth (III) compound with quinolone family member (ciprofloxacin). Antibacterial study. J. Inorg. Biochem. 1997, 66, 241-245.

127. Turel, I.; Golić, L.; Bukovec, P.; Gubina, M. antibacterial tests of bismuth(III)-quinolone (ciprofoxacin, cf ) compounds against Helicobacter pylori and some other bacteria. Crystal structure of $\left(\mathrm{cfH}_{2}\right)_{2}\left[\mathrm{Bi}_{2} \mathrm{Cl}_{10}\right] \cdot 4 \mathrm{H}_{2} \mathrm{O}$. J. Inorg. Biochem. 1998, 71, 53-60. 
128. Turel, I.; Guber, K.; Leban, I.; Bukovec, N. Synthesis, crystal structure, and characterization of tree novel compounds of quinolone family member (norfloxacin). J. Inorg. Biochem. 1996, 61, 197-212.

129. Vasiliev, D.; Golovnev, N.N. Synthesis and Structure of $\mathrm{C}_{17} \mathrm{H}_{22} \mathrm{FN}_{3} \mathrm{O}_{3}{ }^{2+} \mathrm{CuCl}_{4}{ }^{2-}$. J. Struct. Chem. 2010, 51, 177-180.

130. Turel, I.; Leban, I.; Klinchar, G.; Bukovec, N.; Zalar, S. Synthesis, crystal structure and characterization of two metal-quinolone compound. J. Inorg. Biochem. 1997, 66, 77-82.

131. Zupančič, M.; Turel, I.; Bukovec, P.; White, A.J.P.; Williams, D.J. Synthesis and characterization of 2 novel zinc (II) complexes with ciprofloxacin. Crystal-structure of $\left[\mathrm{C}_{17} \mathrm{H}_{19} \mathrm{~N}_{3} \mathrm{O}_{3} \mathrm{~F}\right]_{2} \cdot\left[\mathrm{ZnCl}_{4}\right] \cdot 2 \mathrm{H}_{2} \mathrm{O}$. Croat. Chem. Acta 2001, 74, 61-74.

132. Polishchuk, A.V.; Karaseva, E.T.; Cherednichenko, A.I.; Gerasimenko, A.V.; Karasev, V.E. Crystal structure and X-ray photoelectron spectroscopy of ciprofloxacinium tetrachloroaurate monohydrate. Russ. J. Coord. Chem. 2011, 37, 215-222.

133. Olivera, M.E.; Mazzieri, M.R.; Manzo, R.H. New pharmaceutical fluoroquinolone derivatives hydrochloride of aluminum complexes of ciprofloxacin and norfloxacin. STP Pharma Sci. 2000, $10,251-256$.

134. Olivera, M.E.; Allemandi, D.A.; Manzo, R.H. Intrinsic dissolution rate and intestinal permeability of metallic complexes of norfloxacin and ciprofloxacin in relation to their formulation. Acta Farm. Bonaerense 2000, 19, 185-191.

135. Alovero, F.L.; Olivera, M.E.; Manzo, R.H. In vitro pharmacodynamic properties of a fluoroquinolone pharmaceutical derivative: Hydrochloride of ciprofloxacin-aluminium complex. Int. J. Antimicrob. Agents 2003, 21, 446-451.

136. Höffken, G.; Borner, K.; Glatzel, P.D.; Koeppe, P.; Lode, H. Reduced enteral absorption of ciprofloxacin in the presence of antacids (letter). Eur. J. Clin. Microbiol. 1985, 4, 345.

137. Kara, M.; Hassinoff, B.B.; Mckay, D.N.; Campbell, N.R.C. Clinical and chemical interactions between iron preparations and ciprofloxacin. Brit. J. Clin. Pharmacol. 1991, 31, 257-261.

138. Wallis, S.C.; Charles, B.G.; Gahan, L.R.; Filippich, L.J.; Bredhauer, M.G.; Duckworth, P.A. Interaction of norfloxacin with divalent and trivalent pharmaceutical cations, In vitro complexation and in vivo pharmacokinetic studies in the dog. J. Pharm. Sci. 1996, 85, 803-809.

139. Davies, M.; Maesen, F.P.V. Drug interactions with quinolones. Rev. Infect. Dis. 1989, 2 , S1083-S1090.

140. Nix, D.E.; Watson, W.A.; Handy, L.; Frost, R.W.; Rescott, D.L.; Goldstein, H.R. The effect of sucralfate pretreatment on the pharmacokinetics of ciprofloxacin. Pharmacotherapy 1989, 9, 377-380.

141. Polk, R.E.; Healey, D.P.; Sahai, J.; Drwal, L.; Racht, E. Effect of ferrous sulphate and multivitamins with zinc on absorption of ciprofloxacin in normal volunteers. Antimicrob. Agents Chemother. 1989, 33, 1841-1844.

142. Ross, D.; Riley, C. Physicochemical properties of the fluoroquinolone antimicrobials. III. Complexation of lomefloxacin with various metal ions and the effect of metal ion complexation on aqueous solubility. Int. J. Pharmaceut. 1992, 87, 203-213. 
143. Ross, D.; Elkinton, S.; Knaub, S.; Riley, C. Physicochemical properties of the fluoroquinolone antimicrobials. VI. Effect of metal-ion complexation on octanol-1-ol-water partitioning. Int. J. Pharmaceut. 1993, 93, 131-138.

144. Žakelj, S.; Berginc, K.; Uršič, D.; Veber, M.; Kristl, A. Metal cation-fluoroquinolone complexes do not permeate through the intestinal absorption barrier. J. Pharm. Biomed. Anal. 2010, 53, $655-659$.

145. Pallù, G.; Valisena, S.; Ciarrocchi, G.; Gatto, B.; Palumbo, M. Quinolone binding to DNA is mediated by magnesium ions. Proc. Natl. Acad. Sci. USA 1992, 89, 9671-9675.

146. Skauge, T.; Turel, I.; Sletten, E. Interaction between ciprofloxacin and DNA mediated by $\mathrm{Mg}^{2+}$-ions. Inorg. Chim. Acta 2002, 339, 239-247.

147. Song, G.; Yan, Q.; He, Y. Studies on interaction of norfloxacin, $\mathrm{Cu}^{2+}$ and DNA by spectral methods. J. Fluoresc. 2005, 15, 673-678.

148. Drevenšek, P.; Turel, I.; Poklar Ulrih, N. Influence o copper (II) and magnesium (II) ions on the ciprofloxacin binding to DNA. J. Inorg. Biochem. 2003, 96, 407-415.

149. Guo, D.-S.; Jing, B.Y.; Yuan, X.-Y. Influence of $\mathrm{Mg}^{2+}$ and $\mathrm{Cu}^{2+}$ on the interaction between quinolone and calf thymus DNA. J. Fluoresc. 2011, 21, 113-118.

150. Song, G.; He, Y.; Cai, Z. The interaction between levofloxacine hydrochloride and DNA mediated by $\mathrm{Cu}^{2+}$. J. Fluoresc. 2004, 14, 705-710.

151. Yuan, X.-Y.; Qin, J.; Lu, L.-L. Influence of metal ions on the interaction between gatifloxacin and calf thymus DNA. Spectrochim. Acta A 2010, 75, 520-524.

152. Yuan, X.-Y.; Guo, D.-S.; Wang, L.L. Influence of $\mathrm{Mg}^{2+}$ and $\mathrm{Cd}^{2+}$ on the interaction between sparfloxacin and calf thymus DNA. Spectrochim. Acta A 2008, 69, 1130-1135.

153. Guo, D.-S.; Yuan, X.-Y.; Wu, J.-B. Influence of $\mathrm{Cr}(\mathrm{III})$ and $\mathrm{Cr}(\mathrm{VI})$ on the interaction between sparfloxacin and calf thymus DNA. J. Inorg. Biochem. 2007, 101, 644-648.

154. Zhang, G.; Fu, X.; Liu, Q.; Wang, G. Interaction between pazufloxacin and DNA mediated by copper(II) ions. J. Fluoresc. 2008, 18, 701-706.

155. Sissi, C.; Andreolli, M.; Cecchetti, V.; Fravolini, A.; Gatto, B.; Palumbo, M. $\mathrm{Mg}^{2+}$-mediated binding of 6-Substituted quinolones to DNA: Relevance to biological activity. Bioorg. Med. Chem. 1998, 6, 1555-1561.

156. Robles, J.; Martin-Polo, J.; Avarez-Valtierra, L.; Hinojosa, L.; Mendoza-Diaz, G. A theoretical-experimental study on the structure and activity of certain quinolones and the interaction of their $\mathrm{Cu}(\mathrm{II})$-complexes on a DNA model. Met. Based Drugs 2000, 7, 301-311.

157. Sissi, C.; Marangon, E.; Chemello, A.; Noble, C.G.; Maxwell, A.; Palumbo, M. The effects of metal ions on the structure and stability of the DNA gyrase B protein. J. Mol. Biol. 2005, 353, 1152-1160.

158. Sissi, C.; Palumbo, M. Effects of magnesium and related divalent metal ions in topoisomerase structure and function. Nucleic Acids Res. 2009, 37, 702-711.

159. Wohlkonig, A.; Chan, P.F.; Fosberry, A.P.; Homes, P.; Huang, J.; Kranz, M.; Leydon, V.R.; Miles, T.J.; Pearson, N.D.; Perera, R.L.; et al. Structural basis of quinolone inhibition of type IIA topoisomerases and target-mediated resistance. Nat. Struct. Mol. Biol. 2010, 17, 1152-1153. 
160. Aldred, K.J.; McPherson, S.A.; Turnbough, C.L., Jr.; Kerns, R.J.; Osheroff, N. Topoisomerase IV-quinolone interactions are mediated through a water-metal ion bridge: Mechanistic basis of quinolone resistance. Nucleic Acids Res. 2013, 41, 4628-4639.

161. Lecomte, S.; Baron, M.H.; Chenon, M.T.; Compry, C.; Moreau, N.J. Effect of magnesium complexation by fluoroquinolones on their antibacterial properties. Antimicrob. Agents Chemother. 1994, 38, 2810-2816.

162. Alkaysi, H.N.; Abdel-Hay, M.H.; Sheikh Salem, M.; Gharaibeh, A.M.; Na'was, T.E. Chemical and biological investigations of metal ion interaction with norfloxacin. Int. J. Pharmaceut. 1992, $87,73-77$.

163. Tumer, M.; Koksal, H.; Sener, M.K.; Serin, S. Antimicrobial activity studies of the binuclear metal complexes derived from tridentate schiff base ligands. Transit. Met. Chem. 1999, 24, 414-420.

164. Imran, M.; Iqbal, J.; Iqbal, S.; Ijaz, N. In vitro antibacterial studies of ciprofloxacin-imines and their complexes with $\mathrm{Cu}(\mathrm{II}), \mathrm{Ni}(\mathrm{II}), \mathrm{Co}(\mathrm{II})$, and Zn(II). Turk. J. Biol. 2007, 31, 67-72.

165. Patel, N.H.; Parekh, H.M.; Patel, M.N. Synthesis, physicochemical characteristics, and biocidal activity of some transition metal mixed-ligand complexes with bidentate (NO and NN) Schiff bases. Pharm. Chem. J. 2007, 41, 78-82.

166. Takiff, H.; Guerrero, E. Current prospects for the fluoroquinolones as first-line tuberculosis therapy. Antimicrob. Agents. Chemother. 2011, 55, 5421-5429.

167. Chang, K.C.; Yew, W.W.; Chan, R.C. Rapid assays for fluoroquinolone resistance in Mycobacterium tuberculosis: A systematic review and meta-analysis. J. Antimicrob. Chemother. 2010, 65, 1551-1561.

168. Ahmad, S.; Mokaddas, E. Recent advances in the diagnosis and treatment of multidrug-resistant tuberculosis. Respir. Med. 2009, 103, 1777-1790.

169. Saha, D.K.; Padhye, S.; Anson, C.E.; Powell, A.K. Hydrothermal synthesis, crystal structure, spectroscopy, electrochemistry and antimycobacterial evaluation of the copper (II) ciprofloxacin complex: $\left[\mathrm{Cu}(\mathrm{cf})_{2}\left(\mathrm{BF}_{4}\right)_{2}\right] \cdot 6 \mathrm{H}_{2} \mathrm{O}$. Inorg. Chem. Commun. 2002, 5, 1022-1027.

170. Sulochana, S.; Rahman, F.; Paramasivan, C.N. In vitro activity of fluoroquinolones against Mycobacterium tuberculosis. J. Chemother. 2005, 17, 169-173.

171. Nishizawa, T.; Suzuki, H.; Hibi, T. Quinolone-based third-line therapy for Helicobacter pylori eradication. J. Clin. Biochem. Nutr. 2009, 44, 119-124.

172. Berning, M.; Krasz, S.; Miehlke, S. Should quinolones come first in Helicobacter pylori therapy? Ther. Adv. Gastroenterol. 2011, 4, 103-114.

173. Malfertheiner, P.; Bazzoli, F.; Delchier, J.C.; Celiñski, K.; Giguère, M.; Rivière, M.; Mégraud, F. Helicobacter pylori eradication with a capsule containing bismuth subcitrate potassium, metronidazole, and tetracycline given with omeprazole versus clarithromycin-based triple therapy: A randomised, open-label, non-inferiority, phase 3 trial. Lancet 2011, 377, 905-913.

174. Ergül, B.; Koçak, E.; Taş, A.; Filik, L.; Köklü, S. Bismuth, moxifloxacin, tetracycline, lansoprazole quadruple first line therapy for eradication of $\boldsymbol{H}$. pylori: A prospective study. Clin. Res. Hepatol. Gastroenterol. 2013, doi:10.1016/j.clinre.2012.10.014. 
175. Turel, I.; Kljun, J.; Perdih, F.; Morozova, E.; Bakulev, V.; Kasyanenco, N.; Byl, J.A.W.; Osheroff, N. First ruthenium organometallic complex of antibacterial agent ofloxacin. Crystal structure and interactions with DNA. Inorg. Chem. 2010, 49, 10750-10752.

176. Herold, C.; Ocker, M.; Ganslmayer, M.; Gerauer, H.; Hahn, E.G.; Schuppan, D. Ciprofloxacin induces apoptosis and inhibits proliferation of human colorectal carcinoma cells. Br. J. Cancer 2002, 86, 443-448.

177. Sissi, C.; Palumbo, M. The quinolone family: From antibacterial to anticancer agents. Curr. Med. Chem. Anticancer Agents. 2003, 3, 439-450.

178. Thadepalli, H.; Salem, F.; Chuah, S.K.; Gollapudi, S. Antitumor activity of trovafloxacin in an animal model. In Vivo 2005, 19. 269-276.

179. Ahmed, A.; Daneshtalab, M. Nonclassical Biological Activities of Quinolone Derivatives. J. Pharm. Pharmaceut. Sci. 2012, 15, 52-72.

180. Rhule, J.T.; Hill, C.L.; Judd D.A.; Schinazi, R.F. Polyoxometalates in medicine. Chem. Rev. 1998, 98, 327357.

181. Kljun, J.; Bytzek, A.K.; Kandioller, W.; Bartel, C.; Jakupec, M.A.; Hartinger, C.G.; Keppler, B.K.; Turel, I. Physicochemical studies and anticancer potency of ruthenium $\eta-p-$ cymene complexes containing antibacterial quinolones. Organometallics 2011, 30, 2506-2512.

182. Eboka, C.J.; Aigbavboa, S.O.; Akerele, J.O. Colorimetric determination of the fluoroquinolones. J. Antimicrob. Chemother. 1997, 39, 639-641.

183. Fratini, L.; Schapoval, E.E.S. Ciprofloxacin determination by visible light spectrophotometry using iron(III) nitrate. Int. J. Pharmaceut. 1996, 127, 279-282.

184. Sultan, S.M.; Suliman, F.-E.O. Chemometric optimization and flow injection method for the determination of norfloxacin in drug formulations. Analyst 1993, 118, 573-576.

185. Al-Momani, I.F.; Haj-Hussein, A.T.; Tahtamouni, A.N. Flow injection spectrophotometric and chromatographic determination of ciprofloxacin and norfloxacin in pharmaceutical formulations, J. Flow Inject. Anal. 2008, 25, 151-155.

186. García, M.S.; Albero, M.I.; Sánchez-Pedreño, C.; Abuherba, M.S. Flow injection spectrophotometric determination of ofloxacin in pharmaceuticals and urine. Eur. J. Pharm. Biopharm. 2005, 61, 87-93.

187. Sultan, S.M.; Suliman, F.-E.O. Flow injection spectrophotometric determination of the antibiotic ciprofloxacin in drug formulations. Analyst 1992, 117, 1523-1526.

188. Suliman, F.E.O; Sultan, S.M. Sequential injection technique employed for stoichiometric studies, optimization and quantitative determination of some fluoroquinolones antibiotics complexed with iron (III) in sulfuric acid media. Talanta 1996, 43, 559-568.

189. El Khateeb, S.Z.; Razek, S.A.; Amer, M.M. Stability-indicating methods for the spectrophotometric determination of norfloxacin. J. Pharm. Biomed. Anal. 1998, 17, 829-840.

190. Rizk, M.; Belal, F.; Ibrahim, F.; Ahmed, S.; Sheribah, Z.A. Derivative spectrophotometric analysis of 4-quinolone antibacterials in formulations and spiked biological fluids by their $\mathrm{Cu}$ (II) complexes. J. AOAC Int. 2001, 84, 368-375.

191. El Walily, A.F.M.; Belal, S.F.; Bakry, R.S. Spectrophotometric and spectrofluorimetric estimation of ciprofloxacin and norfloxacin by ternary complex formation with eosin and palladium(II). J. Pharm. Biomed. Anal. 1996, 14, 561-569. 
192. Yamaguchi, T.; Nakao, M.; Nakahara, R.; Nishioka, Y.; Ikeda, C.; Fujita, Y. Spectrophotometric determination of quinolone antibiotics by an association complex formation with aluminum(III) and erythrosine. Anal. Sci. 2009, 25, 125-128.

193. Uivarosi, V.; Monciu, C.M. The gravimetric and spectrophotometric assay of ofloxacin using ammonium reineckate. Rev. Chim. 2005, 56, 726-730.

194. Uivarosi, V.; Monciu, C.M. Studies on the gravimetric and spectrophotometric analysis of norfloxacin using ammonium reineckate. Rev. Chim. 2009, 60, 132-136.

195. Salem, H. Spectrofluorimetric, atomic absorption spectrometric and spectrophotometric determination of some fluoroquinolones. Am. J. Appl. Sci. 2005, 2, 719-729.

196. Cordoba-Diaz, M.; Cordoba-Borrego, M.; Cordoba-Diaz, D. modification of fluorescent properties of norfloxacin in the presence of certain antacids. J. Pharm. Biomed. Anal. 1998, 18, $565-571$.

197. Rizk, M.; Belal, F.; Ibrahim, F.; Ahmed, S.; el-Enany, N. Spectrofluorimetric analysis of certain 4-quinolone in pharmaceuticals and biological fluids. Pharm. Acta Helv. 2000, 74, 371-377.

198. Djurdjević, P.T.; Jelikić-Stankov, M.; Stankov, D. Fluorescence reaction and complexation equilibria between norfloxacin and aluminium (III) ion in chloride medium. Anal. Chim. Acta 1995, 300, 253-259.

199. Pérez-Ruiz, T.; Martínez-Lozano, C.; Tomás, V. Carpena, J. Determination of norfloxacin in real samples by different pectrofluorimetric techniques. Analyst 1997, 122, 705-708.

200. Han, Y.; Wu, X.; Yang, J.; Sun, S. The fluorescence characteristic of the yttrium-norfloxacin system and its analytical application. J. Pharm. Biomed. Anal. 2005, 38, 528-531.

201. Tong, C.; Zhuo X.; Liu, W.; Wu, J. Synchronous fluorescence measurement of enrofloxacin in the pharmaceutical formulation and its residue in milks based on the yttrium (III)-perturbed luminescence. Talanta 2010, 82, 1858-1863.

202. Beltyukova, S.; Teslyuk, O.; Egorova, A.; Tselik, E. Solid-phase luminescence determination of ciprofloxacin and norfloxacin in biological fluids. J. Fluoresc. 2002, 12, 269-271.

203. Tong, C.; Xiang, G. Sensitive determination of enoxacin by its enhancement effect on the fluorescence of terbium(III)-sodium dodecylbenzene sulfonate and its luminescence mechanism. J. Luminesc. 2007, 126, 575-580.

204. Kaur, K.; Singh Saini, S.; Malik, A.K., Singh, B. Micelle enhanced and terbium sensitized spectrofluorimetric determination of danofloxacin in milk using molecularly imprinted solid phase extraction. Spectrochim. Acta A 2012, 96, 790-795.

205. Zhao, H.C.; Ding F.; Wang, X.; Ju, H.; Li, A.; Jin, L.P. A study on silver nanoparticles-sensitized fluorescence and second-order scattering of the complexes of $\mathrm{Tb}$ (III) with ciprofloxacin and its applications. Spectrochim. Acta Part A 2008, 70, 332-336.

206. Ding, F.; Zhao, H.; Jin, L.; Zheng, D. Study of the influence of silver nanoparticles on the second-order scattering and the fluorescence of the complexes of $\mathrm{Tb}$ (III) with quinolones and determination of the quinolones. Anal. Chim. Acta 2006, 566, 136-143.

207. Attia, M.S.; Essawy, A.A.; Youssef, A.O. Europium-sensitized and simultaneous pH-assisted spectrofluorimetric assessment of ciprofloxacin, norfloxacin and gatifloxacin in pharmaceutical and serum samples. J. Photochem. Photobiol. A 2012, 236, 26-34. 
208. Dong, P.; Na, X.; Fu, B.; Wang, L. Rapid europium-sensitized fluorescent determination of ulifloxacin, the active metabolite of prulifloxacin, in human serum and urine. J. Pharm. Anal. 2011, 1, 46-50.

209. Attia, M.S.; Youssef, A.O.; Essawy, Amr A.; Abdel-Mottaleb, M.S.A. A highly luminescent complexes of $\mathrm{Eu}(\mathrm{III})$ and $\mathrm{Tb}(\mathrm{III})$ with norfloxacin and gatifloxacin doped in sol-gel matrix: A comparable approach of using silica doped $\mathrm{Tb}(\mathrm{III})$ and $\mathrm{Eu}(\mathrm{III})$ as optical sensor. J. Luminesc. 2012, 132, 2741-2746.

210. Jelikić-Stankov, M.; Stankov, D.; Djurdjević, P. Determination of pefloxacin in serum by time-resolved fluorimetry. Pharmazie 1999, 54, 73-74.

211. Luiz, F.C.L.; Garcia, L.S.; Goes Filho, L.S.; Teixeira, L.R.; Louro, S.R.W. Fluorescence studies of gold(III)-norfloxacin complexes in aqueous solutions. J. Fluoresc. 2011, 21, 1933-1940.

212. Pan, B.; Han, X.; Wu, M.; Peng, H.; Zhang, D.; Li, H.; Xing, B. Temperature dependence of ofloxacin fluorescence quenching and complexation by $\mathrm{Cu}(\mathrm{II})$. Environ. Pollut. 2012, 171, 168-173.

213. Zhang, Z.Q.; Jiang, Y.C. Flow injection flame atomic spectrometry for the indirect analysis of norfloxacin, Atom. Spectroscop. 2001, 22, 429-432.

214. Al-Ghannam, S.M. Atomic absorption spectroscopic, conductometric and colorimetric methods for determination of some fluoroquinolone antibacterials using ammonium reineckate. Spectrochim. Acta A 2008, 69, 1188-1194.

215. Issopoulos, B.P. spectrophotometric determination of norfloxacin in pharmaceutical formulations. Analyst 1989, 114, 627-630.

216. Wang, N.-X.; Wang, L.; Jiang, W.; Ren, Y.-Z.; Si, Z.-K.; Qiu, X.-X.; Du, G.-Y.; Qi, P. Determination of neodymium, holmium and erbium in mixed rare earths by norfloxacin. Fresenius J. Anal. Chem. 1998, 361, 821-824.

217. Guo C.; Lang, A.; Wang, L.; Jiang, W. The co-luminescence effect of a europium (III)-lanthanum (III)-gatifloxacin-sodium dodecylbenzene sulfonate system and its application for the determination of trace amount of europium(III). J. Luminesc. 2010, 130, 591-597.

218. Tan, H.; Zhang, Y.; Chen, Y. Detection of mercury ions $\left(\mathrm{Hg}^{2+}\right)$ in urine using a terbium chelate fluorescent probe. Sens. Actuators B 2011, 156, 120-125.

219. Beltyukova, S.V.; Egorova, A.V.; Teslyuk, O.I. Europium(III) and terbium(III) chelates of quinolonecarboxylic acid derivatives as labels for immunofluorimetric assay. J. Anal. Chem. 2000, 55, 682-685.

220. Tong, C.; Hu, Z.; Liu, W. Enoxacin-Tb ${ }^{3+}$ complex as an environmentally friendly fluorescence probe for DNA and its application. Talanta 2007, 71, 816-821.

(C) 2013 by the authors; licensee MDPI, Basel, Switzerland. This article is an open access article distributed under the terms and conditions of the Creative Commons Attribution license (http://creativecommons.org/licenses/by/3.0/). 